\title{
Detailed homogeneous abundance studies of 14 Galactic s-process enriched post-AGB stars: In search of lead $(\mathrm{Pb})^{\star, \star \star, \star \star \star \star}$
}

\author{
K. De Smedt ${ }^{1}$, H. Van Winckel ${ }^{1}$, D. Kamath ${ }^{1}$, L. Siess ${ }^{2}$, S. Goriely ${ }^{2}$, A. I. Karakas ${ }^{3}$, and R. Manick ${ }^{1}$ \\ ${ }^{1}$ Instituut voor Sterrenkunde, KU Leuven, Celestijnenlaan 200D, 3001 Leuven, Belgium \\ e-mail: Hans.VanWinckel@ster . kuleuven.be \\ 2 Institut d'Astronomie et d'Astrophysique, Université Libre de Bruxelles, ULB, CP 226, 1050 Brussels, Belgium \\ 3 Research School of Astronomy and Astrophysics, Australian National University, Canberra, ACT 2611, Australia
}

Received 23 September 2015 / Accepted 4 December 2015

\section{ABSTRACT}

\begin{abstract}
Context. This paper is part of a larger project in which we systematically study the chemical abundances of Galactic and extragalactic post-asymptotic giant branch (post-AGB) stars. The goal at large is to provide improved observational constraints to the models of the complex interplay between the AGB $s$-process nucleosynthesis and the associated mixing processes.

Aims. Lead $(\mathrm{Pb})$ is the final product of the $s$-process nucleosynthesis and is predicted to have large overabundances with respect to other $s$-process elements in AGB stars of low metallicities. However, $\mathrm{Pb}$ abundance studies of $s$-process enriched post-AGB stars in the Magellanic Clouds show a discrepancy between observed and predicted $\mathrm{Pb}$ abundances. The determined upper limits based on spectral studies are much lower than what is predicted. In this paper, we focus specifically on the $\mathrm{Pb}$ abundance of $14 \mathrm{Galactic}$ $s$-process enhanced post-AGB stars to check whether the same discrepancy is present in the Galaxy as well. Among these 14 objects, two were not yet subject to a detailed abundance study in the literature. We apply the same method to obtain accurate abundances for the 12 others. Our homogeneous abundance results provide the input of detailed spectral synthesis computations in the spectral regions where $\mathrm{Pb}$ lines are located.

Methods. We used high-resolution UVES and HERMES spectra for detailed spectral abundance studies of our sample of Galactic post-AGB stars. None of the sample stars display clear $\mathrm{Pb}$ lines, and we only deduced upper limits of the $\mathrm{Pb}$ abundance by using spectrum synthesis in the spectral ranges of the strongest $\mathrm{Pb}$ lines.

Results. We do not find any clear evidence of $\mathrm{Pb}$ overabundances in our sample. The derived upper limits are strongly correlated with the effective temperature of the stars with increasing upper limits for increasing effective temperatures. We obtain stronger $\mathrm{Pb}$ constraints on the cooler objects. Moreover, we confirm the s-process enrichment and carbon enhancement of two unstudied $21 \mu \mathrm{m}$ sources IRAS $13245-6428$ and IRAS 14429-4539. The mildly s-process enhanced post-AGB star IRAS $17279-1119$ is part of a binary system and may be the long sought precursor of extrinsic Ba stars.

Conclusions. Stars with $T_{\text {eff }}>7500 \mathrm{~K}$ do not provide strong constraints on the Pb abundance as the strongest line in the optical spectrum is only detectable at unrealistically high $\mathrm{Pb}$ atmospheric abundances. Combining the $\mathrm{Pb}$ abundance results from this study with abundances from our previous studies, we conclude that the discrepancy between theory and observation increases towards lower metallicities. The model predictions are consistent with the deduced upper limits on the $\mathrm{Pb}$ abundances for all stars with $[\mathrm{Fe} / \mathrm{H}]>-0.7$ dex. For stars with $[\mathrm{Fe} / \mathrm{H}]<-0.7$ dex, however, the model predictions overestimate the $\mathrm{Pb}$ abundances with respect to the other $s$-process elements. All objects, except IRAS 17279-1119, confirm the relation between neutron exposure [hs/ls] and third dredge-up efficiency $[\mathrm{s} / \mathrm{Fe}]$, whereas no relation between metallicity and neutron exposure is detected within the metallicity range of our total sample $(-1.4<[\mathrm{Fe} / \mathrm{H}]<-0.2)$. The mild enrichment of IRAS $17279-1119$ can probably be attributed to a cut-off of the AGB evolution due to binary interactions. To our knowledge, IRAS 17279-1119 is the first $s$-process enhanced Galactic post-AGB star known in a binary system and is a possible precursor of the extrinsic Ba dwarf stars. We corroborate the finding that the variety in abundance profiles shows that a large spread of neutron irradiation is needed for a given metallicity. Lead-rich stars are yet to be found among post-AGB stars.
\end{abstract}

Key words. stars: AGB and post-AGB - stars: abundances - binaries: spectroscopic - stars: evolution

\section{Introduction}

$\star$ Based on observations collected with the Very Large Telescope at the ESO Paranal Observatory (Chili) of programme numbers 066.D0171, 073.D-0241 and 094.D-0067.

$\star \star$ Based on observations made with the Mercator Telescope, operated on the island of La Palma by the Flemish Community, at the Spanish Observatorio del Roque de los Muchachos of the Instituto de Astrofísica de Canarias.

$\star \star \star$ Equivalent width measurements and a copy of the reduced spectra (FITS files) are only available at the CDS via anonymous ftp to cdsarc.u-strasbg.fr (130.79.128.5) or via http://cdsarc.u-strasbg.fr/viz-bin/qcat?J/A+A/587/A6
The final evolution of low- to intermediate-mass single stars $\left(M \lesssim 7 M_{\odot}\right.$ ) is characterised by a fast transition from the asymptotic giant branch (AGB) over the post-AGB track towards the planetary nebula phase. During late stages of AGB evolution, convective thermal pulses (TPs) occur in the intershell, which are possibly followed by third dredge-ups (TDUs) that transport newly synthesised material from the stellar interior to the surface. The main dredged-up element is ${ }^{12} \mathrm{C}$ as primary product of the triple alpha reaction. AGB stars are thought to be very important contributors to the total carbon and nitrogen enrichment of galaxies (e.g. Romano et al. 2010; Kobayashi et al. 2011). 
Apart from ${ }^{12} \mathrm{C}$, the TDU also brings elements created by neutron synthesis to the stellar surface. The slow-neutron capture process (or $s$-process) is at the origin of approximately half of all cosmic abundances past the iron peak. It is generally acknowledged that the ${ }^{13} \mathrm{C}(\alpha, \mathrm{n}){ }^{16} \mathrm{O}$ reaction is the main neutron source in low-mass AGB stars (1-3 $M_{\odot}$ ) (e.g. Straniero et al. 1995; Gallino et al. 1998; Mowlavi et al. 1998; Abia et al. 2002; Karakas \& Lattanzio 2014; Neyskens et al. 2015). It is also widely accepted that a ${ }^{13} \mathrm{C}$-pocket is produced by the transport of protons from the convective envelope into the He-rich intershell. The neutrons created in the ${ }^{13} \mathrm{C}$-pocket by the alpha capture reaction can then be used for the creation of heavy elements by the slow neutron capture process (s-process). Although observations confirm that heavy elements can indeed be created by AGB stars, the physical mechanisms behind the AGB internal nucleosynthesis and associated dredge-up processes are poorly understood.

Along the valley of stability, the isotopes with the lowest cross-section for neutron capture are located around the nuclei with the magic number $N=50$ (the light $s$-elements or ls) and $N=80$ (the heavy $s$-elements or hs). The end product of the $s$-process nucleosynthesis chain is the doubly magic ${ }^{208} \mathrm{~Pb}$ isotope. The ratio [hs/ls] is an intrinsic indicator of the total neutron irradiation. The $s$-process chain is limited to the ls elements for moderate irradiations, while the hs elements are being produced for increasing neutron irradiation. When the neutron irradiation increases even further, the elements beyond the hs peak are produced up to the final product $\mathrm{Pb}$. As the neutron production is thought to be largely independent of the initial metallicity, there are more neutrons available per iron seed and, hence at low metallicity $\mathrm{Pb}$ is predicted to have large overabundances with respect to other $s$-elements (see e.g. Gallino et al. 1998; Goriely \& Mowlavi 2000; Lugaro et al. 2012, and references therein).

These $\mathrm{Pb}$ abundance predictions are confirmed in some low metallicity extrinsically enriched metal-poor objects, where strong $\mathrm{Pb}$ enhancements are indeed observed (e.g. Van Eck et al. 2001, 2003; Behara et al. 2010). These objects are polluted with matter from an evolved binary companion when it passed the TP-AGB phase but that is now a dim white dwarf. However, not all metal-deficient objects with $s$-process enrichment show this strong $\mathrm{Pb}$ overabundance (e.g. Aoki et al. 2001; Van Eck et al. 2003; Bisterzo et al. 2012). A very wide range of neutron irradiations are needed at a given metallicity to explain the abundance spread of extrinsically enhanced low metallicity objects (Bisterzo et al. 2010), the physical orgin of which is not clear. A complicating factor in modelling the extrinsically enhanced objects is that the original enriched AGB star is now a cool white dwarf that is not detected.

Here we focus on intrinsically enriched objects. The AGB photospheres themselves are dominated by molecular transitions that hinder the study of individual elements (e.g. Abia et al. 2008). This problem does not occur in post-AGB stars, for which the photospheres are hotter and are dominated by atomic transitions. The spectra of post-AGB stars allow for extensive chemical studies of individual elements. Post-AGB atmospheres display the outcome of chemical enrichment from internal nucleosynthesis and dredge-up processes during the entire stellar evolution. This makes post-AGB stars ideal probes to study AGB nucleosynthesis (Van Winckel 2003).

In our recent studies of post-AGB stars in the Magellanic Clouds (van Aarle et al. 2011; Kamath et al. 2014, 2015), we focused on the intrinsic $s$-process enriched post-AGB star J004441.04-732136.4 (J004441) in the Small Magellanic Cloud (SMC; De Smedt et al. 2012, 2014). In De Smedt et al. (2014), we compared the observed abundance results with fine-tuned, theoretical state-of-the-art AGB models. Although this star is metal-poor and strongly $s$-process enriched, we found a strong discrepancy between the observed and predicted $\mathrm{Pb}$ overabundance. The best-fitting model overestimates the $\mathrm{Pb}$ overabundance by more than $2 \mathrm{dex}$. Moreover, the same $\mathrm{Pb}$ discrepancy was detected in three other metal-deficient post-AGB stars in the LMC (De Smedt et al. 2014). The extreme overabundances in combination with a negative $[\mathrm{Pb} / \mathrm{hs}]$ value may point to a neutron irradiation that is different from the currently accepted AGB $s$-process scenarios where the neutron irradiation occurs in the radiative layers of the intershell in between thermal pulses. One possible alternative is proton ingestion directly into the convective thermal pulse, but preliminary model calculations (Lugaro et al. 2015) are as yet inconclusive and not able to model the full abundance ratios of $\mathrm{J} 004441$.

This low $\mathrm{Pb}$ content was also detected by Reyniers et al. (2007a) for the LMC star MACHO 47.2496.8. For all of these objects with intrinsic enrichment, the upper limits of the $\mathrm{Pb}$ abundances are equal or smaller than the overabundances of the other $s$-elements. In our most recent study (De Smedt et al. 2015), we found a similar discrepancy for two newly identified $s$ process enriched post-AGB stars in the LMC. We concluded that the low $\mathrm{Pb}$ abundance seems to be a common feature in $s$-process rich, post-AGB stars in the Magellanic Clouds. Furthermore, we find that all the objects studied until now have low initial masses and low metallicities. Therefore, the low observed $\mathrm{Pb}$ abundances strongly contradict theoretical nucleosynthetic predictions of $\mathrm{Pb}$.

In this contribution, we extend our $\mathrm{Pb}$ studies and focus on Galactic $s$-process rich objects. We present a homogeneous study of the neutron irradiation as traced by the $s$-process element distribution of 14 post-AGB stars. We especially focus on the $\mathrm{Pb}$ abundances. In combination with the studied Magellanic Cloud objects, we aim at a systematic study of the distribution of $s$-elements detected in post-AGB stars, which cover a wide range of metallicities. We provide new constraints for the models of AGB nucleosynthesis and associated processes.

The selection of our Galactic sample stars and the observations are described in Sect. 2. The spectral analyses are discussed in Sect. 3 followed by the abundance results of all elements lighter than $\mathrm{Pb}$ in Sect. 4. The abundance results of $\mathrm{Pb}$ are presented in Sect. 5. The neutron irradiation of our sample of stars is discussed in Sect. 6. In Sect. 7, we specifically focus on the evolutionary status of one of the sample stars, IRAS 172791119. We end this paper with the conclusions in Sect. 8.

\section{Sample selection and observations}

Our sample consists of 12 Galactic post-AGB stars, which are known to be $s$-process rich, and two new post-AGB candidates, IRAS 13245-5036 and IRAS 14429-4539, for which no abundance studies have been reported yet. The majority of the already studied Galactic post-AGB stars are $21 \mu \mathrm{m}$ objects, named for the strong solid-state feature at $21 \mu \mathrm{m}$ (Kwok et al. 1989). To date, all $21 \mu \mathrm{m}$ objects are acknowledged to be post-AGB stars with carbon and $s$-process enhancements in their photospheres (e.g. Hrivnak et al. 2009). Since both IRAS 13245-5036 and IRAS 14429-4539 are post-AGB candidates with a clear $21 \mu \mathrm{m}$ feature (Cerrigone et al. 2011), we added these stars to our sample.

We use high-resolution spectra of two different spectrographs. The first spectrograph is the Ultraviolet and Visual Echelle Spectrograph (UVES; Dekker et al. 2000), the echelle 
K. De Smedt et al.: Pb in Galactic s-process enriched post-AGB stars

Table 1. Overview of the sample: name(s), observational logs, references of previous studies, and radial velocities.

\begin{tabular}{|c|c|c|c|c|c|c|c|}
\hline IRAS & $\begin{array}{l}\text { Other } \\
\text { name }\end{array}$ & Date & $\begin{array}{c}\mathrm{UT} \\
\text { start }\end{array}$ & $\begin{array}{l}\text { Exp. time } \\
(\mathrm{s})\end{array}$ & $\begin{array}{c}\text { Telescope }+ \\
\text { spectrograph }\end{array}$ & References & $\begin{array}{c}r_{\mathrm{v}} \\
\left(\mathrm{km} \mathrm{s}^{-1}\right)\end{array}$ \\
\hline $05113+1347^{d}$ & & $\begin{array}{l}2014-10-07 \\
2014-11-23\end{array}$ & $\begin{array}{l}07: 55 \\
05: 03\end{array}$ & $\begin{array}{l}\text { Blue: } 1 \times 2676 \\
\text { Blue: } 1 \times 2676 \\
\text { Red: } 2 \times 1200\end{array}$ & $\begin{array}{l}\text { VLT + UVES } \\
\text { VLT + UVES } \\
\text { VLT + UVES }\end{array}$ & 1 & $\begin{array}{l}6 \pm 2 \\
5 \pm 1 \\
5 \pm 1 \\
\end{array}$ \\
\hline $05341+0852^{d}$ & & $\begin{array}{l}2014-10-06 \\
2014-10-06\end{array}$ & $\begin{array}{l}07: 52 \\
08: 28\end{array}$ & $\begin{array}{l}\text { All: } 1 \times 1826 \\
\text { All: } 1 \times 1826\end{array}$ & $\begin{array}{l}\text { VLT + UVES } \\
\text { VLT + UVES }\end{array}$ & 2 & $\begin{array}{l}28 \pm 1 \\
28 \pm 1\end{array}$ \\
\hline $06530-0213$ & & $\begin{array}{l}2014-12-24 \\
2014-12-24\end{array}$ & $\begin{array}{l}04: 03 \\
04: 50\end{array}$ & $\begin{array}{l}\text { All: } 1 \times 2427 \\
\text { All: } 1 \times 2427\end{array}$ & $\begin{array}{l}\text { VLT + UVES } \\
\text { VLT + UVES }\end{array}$ & 3 & $\begin{array}{l}52 \pm 1 \\
52 \pm 1\end{array}$ \\
\hline $07134+1005^{d}$ & HD 56126 & $\begin{array}{l}2014-09-05 \\
2014-09-08\end{array}$ & $\begin{array}{l}05: 50 \\
05: 39\end{array}$ & $\begin{array}{l}1 \times 920 \\
1 \times 1000\end{array}$ & $\begin{array}{l}\text { Mercator + HERMES } \\
\text { Mercator + HERMES }\end{array}$ & 2 & $\begin{array}{l}92 \pm 1 \\
94 \pm 1\end{array}$ \\
\hline $07430+1115$ & & $2014-12-24$ & $05: 33$ & $\begin{array}{l}\text { Blue: } 1 \times 2176 \\
\text { Red: } 2 \times 1000 \\
\end{array}$ & $\begin{array}{l}\text { VLT + UVES } \\
\text { VLT + UVES } \\
\end{array}$ & 4 & $\begin{array}{l}41 \pm 2 \\
41 \pm 1 \\
\end{array}$ \\
\hline $08143-4406^{b}$ & & $\begin{array}{l}2001-01-16 \\
2001-01-16 \\
2001-02-01\end{array}$ & $\begin{array}{l}06: 41 \\
07: 16 \\
04: 21\end{array}$ & $\begin{array}{c}\text { Blue437: } 2 \times 1800 \\
\text { Red860: } 3 \times 500 \\
\text { Red: } 1 \times 1800\end{array}$ & $\begin{array}{l}\text { VLT + UVES } \\
\text { VLT + UVES } \\
\text { VLT + UVES }\end{array}$ & 3 & $\begin{array}{l}52 \pm 2 \\
52 \pm 1 \\
52 \pm 1\end{array}$ \\
\hline $08281-4850$ & & $\begin{array}{l}2015-01-25 \\
2015-01-25 \\
2015-01-31 \\
\end{array}$ & $\begin{array}{l}04: 18 \\
03: 47 \\
02: 39 \\
\end{array}$ & $\begin{array}{l}\text { All: } 1 \times 2400 \\
\text { All: } 1 \times 2600 \\
\text { All: } 1 \times 2116 \\
\end{array}$ & $\begin{array}{l}\text { VLT + UVES } \\
\text { VLT + UVES } \\
\text { VLT + UVES }\end{array}$ & 5 & $\begin{array}{l}117 \pm 1 \\
116 \pm 1 \\
117 \pm 1 \\
\end{array}$ \\
\hline $13245-5036^{d}$ & & 2015-03-06 & $04: 53$ & All: $1 \times 346$ & VLT + UVES & & $54 \pm 1$ \\
\hline $14325-6428^{c}$ & & $\begin{array}{l}2004-05-13 \\
2004-05-13\end{array}$ & $\begin{array}{l}07: 13 \\
06: 39\end{array}$ & $\begin{array}{c}\text { Blue437: } 1 \times 1800 \\
\text { Red860: } 1 \times 1800 \\
\text { Red: } 1 \times 1800\end{array}$ & $\begin{array}{l}\text { VLT + UVES } \\
\text { VLT + UVES } \\
\text { VLT + UVES }\end{array}$ & 5 & $\begin{array}{l}-83 \pm 1 \\
-83 \pm 1 \\
-82 \pm 1\end{array}$ \\
\hline $14429-4539^{b, d}$ & & $\begin{array}{l}2001-02-03 \\
2001-02-06 \\
2001-02-12\end{array}$ & $\begin{array}{l}08: 24 \\
08: 55 \\
07: 53 \\
07: 54\end{array}$ & $\begin{array}{r}\text { Red: } 1 \times 1800 \\
\text { Red: } 1 \times 1200 \\
\text { Red: } 1 \times 1800 \\
\text { Blue437: } 3 \times 2000 \\
\text { Red860: } 3 \times 2000\end{array}$ & $\begin{array}{l}\text { VLT + UVES } \\
\text { VLT + UVES } \\
\text { VLT + UVES } \\
\text { VLT + UVES } \\
\text { VLT + UVES }\end{array}$ & & $\begin{array}{l}0 \pm 2 \\
0 \pm 2 \\
0 \pm 2 \\
4 \pm 2 \\
4 \pm 2\end{array}$ \\
\hline $17279-1119$ & HD158616 & 2014-09-18 & $00: 00$ & All: $1 \times 65$ & VLT + UVES & 6 & $62 \pm 1$ \\
\hline $19500-1709^{d}$ & HD187885 & 2009-07-16 & 00:20 & $2 \times 2000$ & Mercator + HERMES & 2 & $15 \pm 1$ \\
\hline $22223+4327^{d}$ & V448 Lac & 2009-07-31 & $02: 50$ & $4 \times 1800$ & Mercator + HERMES & 2,6 & $-41 \pm 1$ \\
\hline $22272+5435^{d}$ & HD235858 & 2014-08-29 & $01: 55$ & $3 \times 720$ & Mercator + HERMES & 1 & $-38 \pm 1$ \\
\hline
\end{tabular}

Notes. ${ }^{(a)}$ Exposure times of UVES spectra are split up into three categories: exposure times for the Blue arm, Red arm, or both arms; the latter is indicated with "All". Terms "Blue" and "Red" refer to observations with the Blue390 and Red580 setting. For older observations, the Blue437 and Red860 settings have also been used. ${ }^{(b)}$ For more information about these observations, see Reyniers (2002). ${ }^{(c)}$ For more information about the observations of IRAS 14325-6428, see Reyniers et al. (2007b). ${ }^{(d)}$ Identified $21 \mu \mathrm{m}$ source.

References. 1. Reddy et al. (2002); 2. Van Winckel \& Reyniers (2000); 3. Reyniers et al. (2004); 4. Reddy et al. (1999); 5. Reyniers et al. (2007b); 6. Rao et al. (2012).

spectrograph mounted on the $8 \mathrm{~m}$ UT2 Kueyen Telescope of the VLT array at the Paranal Observatory of ESO in Chili. The second spectrograph is the High Efficiency and Resolution Mercator Echelle Spectrograph (HERMES; Raskin et al. 2011), the spectrograph mounted on the $1.2 \mathrm{~m}$ Mercator telescope at the Roque de los Muchachos Observatory on La Palma.

For all objects, we first checked whether optical highresolution spectra with sufficient signal-to-noise $(\mathrm{S} / \mathrm{N})$ around $4058 \AA$, the spectral region of the strongest identified $\mathrm{Pb}$ I line, were already available for UVES in the ESO archive and for HERMES in the archive of the Institute of Astronomy (KU Leuven). If not, we requested and performed observations for those objects for which a S/N of 25 around $4058 \AA$ would be reached within an hour. All objects, observational details and previous abundance studies are listed in Table 1. The last column shows the radial velocity of the observed spectra.

\subsection{UVES spectra}

The UVES spectra obtained in the period 2014-2015 (see Table 1) were observed using the same setting. We selected the dichroic beam splitter resulting in a wavelength coverage for the blue UVES arm from approximately 3280 to $4530 \AA$, and for the lower and upper part of the mosaic CCD chip from approximately 4780 to $5770 \AA$ and from 5800 to $6810 \AA$, respectively. A slit width of 1 arcsecond was used to gain an optimal compromise between spectral resolution and slit-loss minimisation.

We used the reduced UVES spectra from Reyniers et al. (2004) for our analysis for IRAS 08143-4406. These spectra were observed with different settings, resulting in two sets of spectra representing different wavelength coverages. There is a time gap of 16 days between the observations of both sets of data. This gap was large enough to observe significant changes in the photosphere of IRAS 08143-4406, resulting in two different sets of atmospheric parameters in the analysis by Reyniers et al. (2004). The UVES spectra of post-AGB candidate IRAS 14429-4539 were obtained using the same settings as for IRAS 08143-4406. For details about the observations of IRAS 08143-4406 and IRAS 14429-4539, we refer to Reyniers (2002). For IRAS 14325-6428, we use the observed spectra described in Reyniers et al. (2007b) for the spectral analysis.

All UVES spectra in Table 1, except for IRAS 081434406 and IRAS 14429-4539, were reduced using the UVES pipeline in the Reflex environment of $\mathrm{ESO}^{1}$. The reduction

1 https://www.eso.org/sci/software/reflex/ 


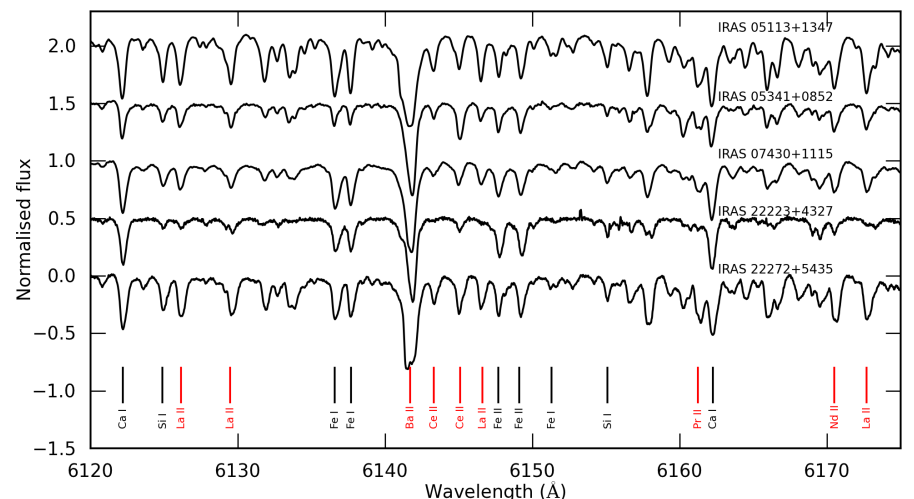

Fig. 1. Comparison of the normalised spectra of all sample stars with $T_{\text {eff }}<7000 \mathrm{~K}$. The spectra have been shifted in flux for clarity and all spectra are shifted to zero velocity. Red and black vertical lines indicate positions of $s$ nuclei and non $s$ nuclei, respectively. For more information, see text.

scheme includes extraction of frames, determinations of wavelength calibration and applying this scale to flat-field divided data. Cosmic clipping was also included. We chose the standard reduction parameters of the UVES pipeline as these gave the best $\mathrm{S} / \mathrm{N}$ of the final spectrum. The spectra of IRAS 08143-4406 and IRAS 14429-4539 were already reduced with older versions of the UVES pipeline.

\subsection{HERMES spectra}

All HERMES spectra were obtained in high-resolution mode $(\lambda / \Delta \lambda=85000)$. These spectra cover a wavelength range from about $3770 \AA$ up to about $9000 \AA$, although some gaps in the spectra are present at large wavelengths, as the reddest orders are too long for the CCD. The specific reduction pipeline of HERMES (Raskin et al. 2011) includes similar steps as the UVES pipeline and is used for the full reduction of the spectra.

\subsection{Normalisation and merging}

When necessary, the individual reduced spectra of each object are first corrected for radial velocity differences (see Table 1). The radial velocities are determined for UVES spectra using the positions of individual spectral lines. The radial velocities are determined for HERMES spectra using a cross-correlation routine specific to the HERMES pipeline (Raskin et al. 2011).

Thereafter, weighted mean spectra are calculated for the individual wavelength ranges of UVES and for the total HERMES spectra. For the normalisation of the spectra, we subdivided the weighted mean spectra into fixed wavelength ranges of $120 \AA$ where the first and last $10 \AA$ overlap with the previous and subsequent spectral parts. Specific care was taken to conserve the Balmer profiles during the normalisation procedure. Each subspectrum is normalised individually by fitting a fifth order polynomial through interactively defined continuum points. After the individual normalisations, all normalised subspectra are then merged into one large spectrum, which is used for the spectral abundance studies.

\section{Spectral analyses}

Figures 1-3 presents the spectral region around the Ba II line at $6141.813 \AA$ together with the identification of several spectral

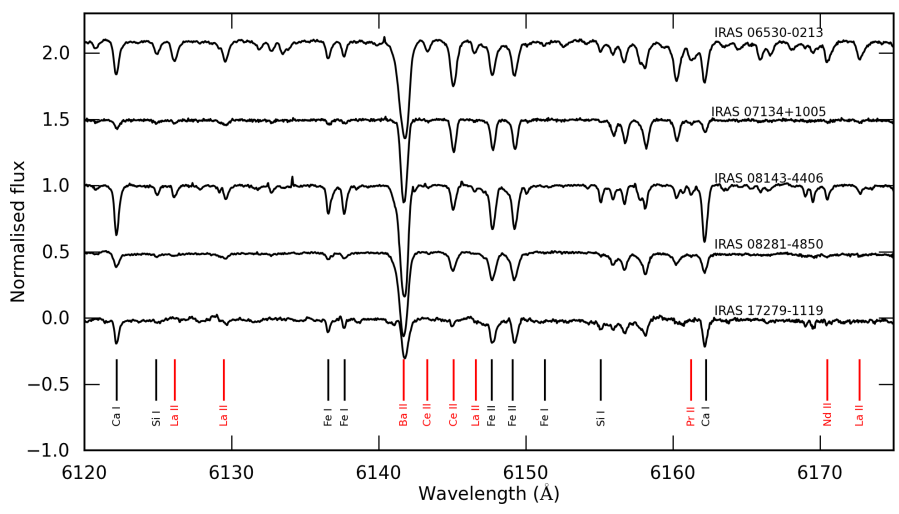

Fig. 2. Similar to Fig. 1, except for the sample stars with $7000 \mathrm{~K} \leqslant$ $T_{\text {eff }}<8000 \mathrm{~K}$.

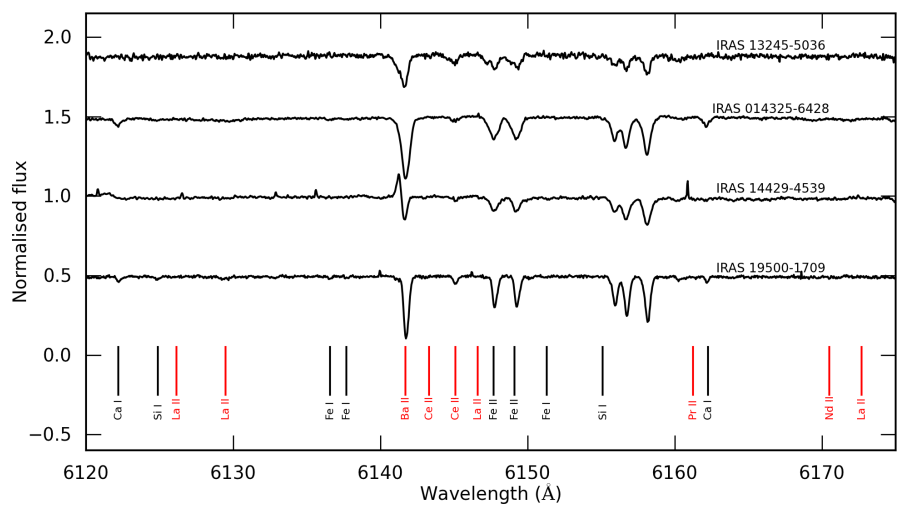

Fig. 3. Similar to Figs. 1 and 2, except for the sample stars with $T_{\text {eff }} \geqslant$ $8000 \mathrm{~K}$.

lines. Each figure corresponds to a different range in effective temperature $T_{\text {eff }}$ (see Sect. 3.1). Figure 1 shows our sample stars with $T_{\text {eff }}<7000 \mathrm{~K}$, Fig. 2 shows sample stars with $7000 \mathrm{~K} \leqslant T_{\text {eff }}<8000 \mathrm{~K}$, and Fig. 3 shows the sample stars with $T_{\text {eff }} \geqslant 8000 \mathrm{~K}$. The figures illustrate how the number of strong spectral lines decreases with increasing effective temperature for all elements.

The overall S/N of the red spectra of UVES (see Table 1) and the optical spectral regions of HERMES is very high and ranges from 80 to 200 . We perform a comparative systematic analysis of all objects and hence decide not to rely on the previous results in the literature, but perform an independent full spectral analysis using the same method for all objects. Depending on the temperature of the star, the $\mathrm{S} / \mathrm{N}$ of spectra below $3700 \AA$ are too poor for both spectrographs, so these regions are not used for our analysis. For the UVES data, we use the full red spectral region sampled in our setting as well as the spectral region from $3900 \AA$ redwards. For hotter stars, we could extend this region towards bluer wavelengths.

In this contribution we focus on $\mathrm{Pb}$ but unfortunately, the optical spectrum of $\mathrm{Pb}$ is poor in spectral lines for the typical atmospheric parameters of our sample stars. The strongest $\mathrm{Pb}$ I lies in the blue part of the spectrum ( $\lambda 4057.807 \AA$ ), a spectral region with low $\mathrm{S} / \mathrm{N}$ and a large number of blends. For some hotter stars, the strongest line of $\mathrm{Pb}$ II at $5608.853 \AA$ is used to constrain the $\mathrm{Pb}$ abundance. To investigate the possible strength of $\mathrm{Pb}$, we calculated which input $[\mathrm{Pb} / \mathrm{H}]$ abundance is needed to create a spectral line of $5 \mathrm{m \AA}$. This value is the lower limit for distinguishing spectral lines from noise in the spectra. Figure 4 shows these theoretical $\mathrm{Pb}$ abundances for different effective 
Table 2. Model atmospheres of the sample of stars.

\begin{tabular}{lccccccc}
\hline \hline Object & $\begin{array}{c}T_{\text {eff }}(\mathrm{K}) \\
\pm 125\end{array}$ & $\begin{array}{c}\log g(\mathrm{dex}) \\
\pm 0.25\end{array}$ & $\begin{array}{c}\xi_{\mathrm{t}}\left(\mathrm{km} \mathrm{s}^{-1}\right) \\
\pm 0.25\end{array}$ & {$[\mathrm{FeI} / \mathrm{H}]$} & {$[\mathrm{FeII} / \mathrm{H}]$} & $N_{\text {FeI }}$ & $N_{\text {FeII }}$ \\
\hline IRAS 05113+1347 & 5500 & 0.50 & 5.00 & $-0.49 \pm 0.15$ & $-0.54 \pm 0.17$ & 21 & 11 \\
IRAS 05341+0852 & 6750 & 1.25 & 3.50 & $-0.70 \pm 0.15$ & $-0.54 \pm 0.11$ & 47 & 20 \\
IRAS 06530-0213 & 7375 & 1.25 & 4.00 & $-0.38 \pm 0.20$ & $-0.32 \pm 0.11$ & 24 & 54 \\
IRAS 07134+1005 & 7250 & 0.50 & 3.25 & $-0.96 \pm 0.31$ & $-0.91 \pm 0.20$ & 42 & 32 \\
IRAS 07430+1115 & 6000 & 1.00 & 3.25 & $-0.31 \pm 0.15$ & $-0.35 \pm 0.15$ & 43 & 17 \\
IRAS 08143-4406 & 7000 & 1.50 & 5.50 & $-0.45 \pm 0.16$ & $-0.43 \pm 0.11$ & 17 & 8 \\
IRAS 08143-4406 & 7250 & 1.50 & 5.00 & $-0.37 \pm 0.17$ & $-0.36 \pm 0.11$ & 70 & 23 \\
IRAS 08281-4850 & 7875 & 1.25 & 5.50 & $-0.29 \pm 0.31$ & $-0.26 \pm 0.11$ & 44 & 23 \\
IRAS 13245-5036 & 9500 & 2.75 & 4.50 & $-0.35 \pm 0.20$ & $-0.30 \pm 0.10$ & 21 & 41 \\
IRAS 14325-6428 & 8000 & 1.00 & 5.75 & $-0.55 \pm 0.33$ & $-0.56 \pm 0.10$ & 26 & 58 \\
IRAS 14429-4539 & 9375 & 2.50 & 4.75 & $-0.26 \pm 0.21$ & $-0.18 \pm 0.11$ & 26 & 52 \\
IRAS 17279-1119 & 7250 & 1.25 & 3.00 & $-0.51 \pm 0.19$ & $-0.64 \pm 0.12$ & 44 & 30 \\
IRAS 19500-1709 & 8000 & 1.00 & 6.00 & $-0.58 \pm 0.42$ & $-0.59 \pm 0.10$ & 33 & 32 \\
IRAS 22223+4327 & 6500 & 1.00 & 4.75 & $-0.32 \pm 0.14$ & $-0.30 \pm 0.11$ & 80 & 25 \\
IRAS 22272+5435 & 5750 & 0.50 & 4.25 & $-0.77 \pm 0.14$ & $-0.77 \pm 0.12$ & 35 & 24 \\
\hline
\end{tabular}

Notes. The errors for $[\mathrm{Fe} / \mathrm{H}]$ include line-to-line scatter and model uncertainty (see Sect. 3.2). $N_{\mathrm{FeI}}$ and $N_{\mathrm{FeII}}$ show the number of lines used for Fe I and Fe II, respectively. ${ }^{(a)}$ Results of the Blue437 and Red860 observations of IRAS 08143-4406 in Table $1 .{ }^{(b)}$ Results of the Red580 observations of IRAS 08143-4406 in Table 1.

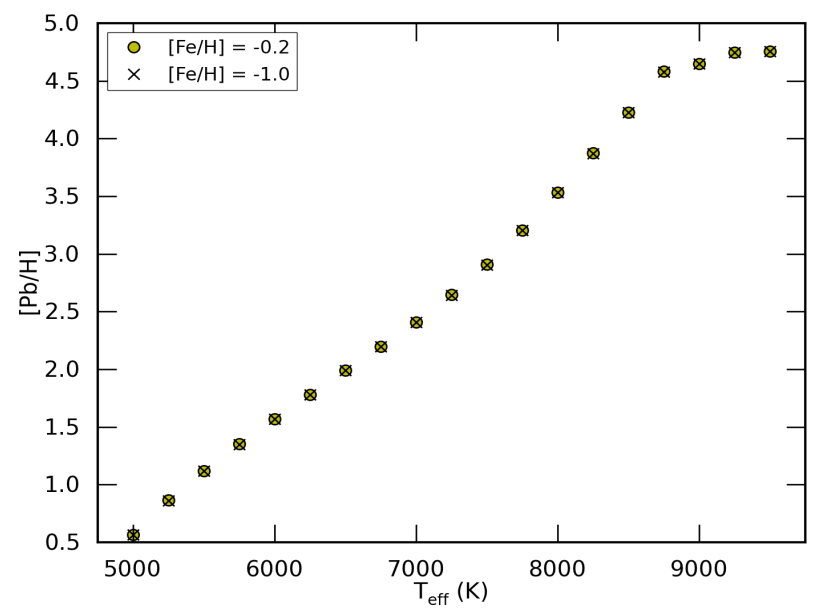

Fig. 4. $[\mathrm{Pb} / \mathrm{H}]$ abundance needed to obtain a $\mathrm{Pb} \mathrm{I}$ or $\mathrm{Pb}$ II spectral line of $5 \mathrm{~mA}$ as a function of effective temperature $T_{\text {eff }}$ and metallicity $[\mathrm{Fe} / \mathrm{H}]$. For more information, see text.

temperatures and for $[\mathrm{Fe} / \mathrm{H}]=-1.0$ dex and $[\mathrm{Fe} / \mathrm{H}]=-0.2$ dex. These abundances are calculated for a $\log g=1.5 \mathrm{dex}$ for all stars with $T_{\text {eff }}<9250 \mathrm{~K}$ and for $\log g=2.0$ dex for 9250 and $9500 \mathrm{~K}$. Figure 4 shows that the needed $\mathrm{Pb}$ abundance for a spectral line detection increases significantly with rising temperature. The figure also shows that the effect of metallicity is negligible in the temperature and metallicity range of the programme stars.

In order to trace $\mathrm{Pb}$ we have to synthesise the spectral regions around the $\mathrm{Pb}$ lines and, hence, we need a complete set of abundances. For these, we used the spectral regions with good $\mathrm{S} / \mathrm{N}$. We use the same methods for the atmospheric parameter determinations and abundance studies for all programme stars. Then, we used these abundances to make detailed spectral synthesis spectra in the regions of the strongest $\mathrm{Pb}$ lines. In this way, we obtained the best constraints on the $\mathrm{Pb}$ abundances.

We have written a Python wrapper (PyMOOG) around the local thermal equilibrium (LTE) abundance calculation routine MOOG (version June 2014 Sneden 1973), which is combined with LTE Kurucz-Castelli atmosphere models (Castelli \& Kurucz 2004). Spectral line identification is based on linelists from the Vienna atomic line database (VALD; Kupka et al. 1999) in combination with a list of spectral lines gathered at the Institute of Astronomy (KU Leuven) for the chemical analysis of A, F, and G stars (Van Winckel \& Reyniers 2000). The implemented linelists cover a wavelength range from 3000 up to $11000 \AA$, covering the full wavelength coverage of the UVES and HERMES spectra. We included linelists for about 160 ions, ranging from helium $(\mathrm{He}, Z=2)$ up to uranium $(U, Z=92)$. Neutral and firstly ionised lines are available for most elements. For some $s$-elements, the second ionisation is also included and is used for the spectral abundance studies of the hottest stars in the sample.

The equivalent width (EW) of spectral lines are interactively measured via direct integration in PyMOOG. The abundances are computed via an iterative process in which the theoretical EWs of single lines are computed for given abundances and matched to the observed EWs. Blended lines are avoided as much as possible, although for some species, $\mathrm{Pb}$ in particular, we sometimes could only use blended lines in our strongly $s$-process enriched stars.

\subsection{Atmospheric parameters}

The atmospheric parameters are determined using $\mathrm{Fe} I$ and Fe II lines in the standard spectroscopic method: the effective temperature $\mathrm{T}_{\text {eff }}$ is determined by imposing the iron abundance to be independent of lower excitation potential; surface gravity $\log g$ by imposing ionisation equilibrium between the individual $\mathrm{Fe}$ I and Fe II abundances; microturbulent velocity by imposing the iron abundance to be independent of reduced equivalent width, which we define as $\mathrm{EW} / \lambda$.

To decrease the abundance uncertainty imposed by the step size of the atmospheric parameters, we use linear interpolation to calculate atmospheric models that lie within the parameter steps of the Kurucz-Castelli models. We choose temperature steps of $125 \mathrm{~K}$, surface gravity steps of $0.25 \mathrm{dex}$, and microturbulent velocity steps of $0.25 \mathrm{~km} \mathrm{~s}^{-1}$.

The individual atmospheric parameter results for the sample of stars are listed in Table 2. The shown uncertainties for $[\mathrm{FeI} / \mathrm{H}]$ and $[\mathrm{FeII} / \mathrm{H}]$ include both line-to-line scatter and atmospheric parameter uncertainties as described below in Sect. 3.2. 
Similar to Reyniers et al. (2004), we have determined the atmospheric parameters separately for the different spectra sets of IRAS 08143-4406 (see Sect. 2). Within the time gap between the observations, the spectral lines of IRAS 08143-4406 had changed significantly, resulting in different sets of atmospheric parameters. These changing spectral line shapes are expected as post-AGB stars cross the Population II Cepheid instability strip during their evolution, and, hence, pulsations may strongly change the atmospheric parameters. For the other stars, the spectral lines did not show any visible changes within the time of the observations.

Typically, the uncertainties for Fe I lines are larger than those of Fe II because of the larger sensitivity of Fe I lines to temperature. For the two new $21 \mu \mathrm{m}$ post-AGB stars IRAS 13245-5036 and IRAS 14429-4539, we find higher temperatures and higher surface gravities with respect to the other sample stars, which points to a more evolved phase of the post-AGB evolutionary stage.

\subsection{Abundance determination}

The abundance analysis of the individual programme star is performed with our preferred atmospheric parameter sets listed in Table 2. We only use spectral lines with EWs smaller than $150 \mathrm{~m} \AA$, as stronger lines are saturated. We also avoid spectral lines with EWs smaller than $5 \mathrm{~m} \AA$ as these may be confused with noise in the spectra.

Ideally, we would only use isolated lines but the strong enrichments and/or high metallicities of the programme stars make this difficult. Therefore, we use spectrum synthesis to check if suspicious spectral lines are part of identified blends. For some elements, we could only determine the abundance with spectral synthesis.

The number of spectral lines for the carbon abundance determination varies strongly with temperature, while for oxygen only a limited number of spectral lines is available in the temperature range of the programme stars. For almost all stars, the $\mathrm{O}$ abundances are determined from the high excitation oxygen multiplet at $6156 \AA$ and/or the forbidden oxygen lines at 6300 and $6363 \AA$. These lines are not affected by possible non-LTE effects (see e.g. Kiselman 2002). For some stars with HERMES spectra, we could determine the nitrogen abundances for spectral lines in the wavelength range between 7000 and $9000 \AA$. These lines are known to be non-LTE sensitive, however, and therefore we apply the temperature-sensitive, non-LTE corrections of Lyubimkov et al. (2011) (see Sect. 4).

The number of lines available for elements ranging from sodium $(\mathrm{Na}, Z=11)$ up to sulphur $(\mathrm{S}, Z=16)$ is limited. Therefore, these abundances cannot be determined in all stars. For the iron-peak elements, ranging from calcium $(\mathrm{Ca}, Z=20)$ up to zinc ( $\mathrm{Zn}, Z=30$ ), we find a wide range of elemental abundances for the majority of stars.

In all programme stars, we find spectral lines of $s$-process elements of the strontium peak $(\mathrm{Sr}, Z=38)$ and barium peak ( $\mathrm{Ba}$, $Z=56$ ), respectively known as the light $s$-process (ls) elements and heavy $s$-process (hs) elements. Unfortunately, we could not determine the abundance of $\mathrm{Sr}$ in any object as the available $\mathrm{Sr}$ lines are always strongly saturated. For the other ls elements yttrium $(Y, Z=39)$ and zirconium $(\mathrm{Zr}, Z=40)$, we find useful spectral lines in each object to determine their abundance. For some stars, we also detect one or two lines for molybdenum (Mo, $Z=42$ ).
For the hs elements, we can always determine the abundances of lanthanum ( $\mathrm{La}, Z=57)$ and neodymium $(\mathrm{Nd}, Z=60)$, and for most of the stars, strong cerium $(\mathrm{Ce}, Z=58)$ lines are present. In a few objects, we are able to determine the abundance of Ba with a weak Ba II line. In the other stars, the available $\mathrm{Ba}$ lines are highly saturated. We can also determine the abundances of a range of elements beyond the Ba-peak such as europium (Eu, $Z=63$ ), gadolinium ( $\mathrm{Gd}, Z=64$ ), dysprosium (Dy, $Z=66$ ), erbium (Er, $Z=68$ ), ytterbium (Yb, $Z=70$ ), lutetium ( $\mathrm{Lu}, Z=71$ ), hafnium (Hf, $Z=72$ ), and tungsten (W, $Z=74$ ). Most of the abundances of these elements are determined with spectral synthesis. We present here a very homogeneous determination of these very heavy elements in all objects with the same atomic data and methodology. Despite the fact that the abundances are often determined with only one or a few lines, we consider the abundances reliable. The high abundances are a clear indication that several elements beyond the Ba-peak are also efficiently synthesised in these objects.

\section{Abundance results to $Z<82$ for all sample stars}

The abundance results of the different programme stars are listed in Tables 3 to 6; each table contains stars of similar effective temperatures. The corresponding $[\mathrm{X} / \mathrm{Fe}]$ results are plotted in Figs. 8-10. An overview of the lines used for these analyses can be found in 15 catalogues available at the CDS. The abundance results for $\mathrm{Pb}$ are discussed in Sect. 5.

The total errors $\sigma_{\text {tot }}$ in Tables 3 to 6 are calculated following the methodology of Deroo et al. (2005). The errors due to atmospheric parameter uncertainties are calculated by determining the elemental abundances for atmospheric models with an effective temperature $T_{\text {eff }} \pm 125 \mathrm{~K}$, surface gravity $\log g \pm 0.25 \mathrm{dex}$ and microturbulent velocity $\xi_{\mathrm{t}} \pm 0.25 \mathrm{~km} \mathrm{~s}^{-1}$ with respect to the preferred value. We include microturbulent velocity in our error analysis despite the fact that for most ions, the associated error is only of the order of a few 0.01 dex. However, for some ions for which only relatively strong spectral lines are available like Y II, the error due to microturbulence can increase to about 0.1 dex.

The total uncertainty $\sigma_{\text {tot }}$ on $[\mathrm{X} / \mathrm{Fe}]$ is then the quadratic sum of the error on the mean (the line-to-line scatter $\sigma_{121}$ divided by the square root of the number of lines used), atmospheric parameter uncertainties $\left(\sigma_{T_{\text {eff }}}, \sigma_{\log g}, \sigma_{\xi_{t}}\right)$, and the Fe abundance error $\left(\sigma_{\mathrm{Fe}}\right)$

$$
\sigma_{\text {tot }}=\sqrt{\left(\frac{\sigma_{121}}{\sqrt{N_{\text {ion }}}}\right)^{2}+\left(\sigma_{T_{\text {eff }}}\right)^{2}+\left(\sigma_{\log g}\right)^{2}+\left(\sigma_{\xi_{\mathrm{t}}}\right)^{2}+\left(\frac{\sigma_{\mathrm{Fe}}}{\sqrt{N_{\mathrm{Fe}}}}\right)^{2}} .
$$

We assume an intrinsic uncertainty of 0.2 dex for all elemental abundances, which are determined by only one line or via spectrum synthesis. This error is a substitute of the line-to-line scatter determined for elements with more useful lines. The $[\mathrm{X} / \mathrm{Fe}]$ abundances are calculated using the Fe ion with an ionisation potential closest to the ionisation potential of the studied ion. In other words, if the ionisation potential of an ion is below the ionisation potential of $\mathrm{Fe} \mathrm{I}$, the Fe I abundance is used for calculating $[\mathrm{X} / \mathrm{Fe}]$. If the ionisation potential exceeds the ionisation potential of Fe I, the abundance of Fe II is used instead.

In Tables 3 to 6 , all elemental abundances were doublechecked by comparing each spectral line with synthetic spectra with the determined abundances. Examples of these comparisons are shown in Figs. 5-7. Figure 5 shows a spectrum synthesis check for the determined C abundance of IRAS $22223+4327$. 
K. De Smedt et al.: Pb in Galactic $s$-process enriched post-AGB stars

Table 3. Abundance results of IRAS 05113+1347, IRAS 05341+0852, IRAS 07430+1115, and IRAS 22272+5435.

\begin{tabular}{|c|c|c|c|c|c|c|c|c|c|c|c|c|c|c|c|c|c|c|c|c|c|}
\hline \multirow[b]{2}{*}{ Ion } & \multirow[b]{2}{*}{$\log \epsilon_{\odot}$} & \multicolumn{5}{|c|}{$\begin{array}{c}\text { IRAS } 05113 \\
{[\mathrm{Fe} / \mathrm{H}]=-0.49} \\
T_{\text {eff }}=5500 \mathrm{~K} \\
\log g=0.5 \mathrm{dex}\end{array}$} & \multicolumn{5}{|c|}{$\begin{array}{c}\text { IRAS } 05341 \\
{[\mathrm{Fe} / \mathrm{H}]=-0.54} \\
T_{\text {eff }}=6750 \mathrm{~K} \\
\log g=1.25 \mathrm{dex}\end{array}$} & \multicolumn{5}{|c|}{$\begin{array}{c}\text { IRAS } 07430 \\
{[\mathrm{Fe} / \mathrm{H}]=-0.31} \\
T_{\text {eff }}=6000 \mathrm{~K} \\
\log g=1.00 \mathrm{dex}\end{array}$} & \multicolumn{5}{|c|}{$\begin{array}{c}\text { IRAS } 22272 \\
{[\mathrm{Fe} / \mathrm{H}]=-0.77} \\
T_{\text {eff }}=5750 \mathrm{~K} \\
\log g=0.5 \mathrm{dex}\end{array}$} \\
\hline & & $N$ & $\operatorname{gg} \epsilon$ & $\sigma_{121}$ & {$[\mathrm{X} / \mathrm{Fe}]$} & & $N$ & $\log \epsilon$ & $\sigma_{121}$ & {$[\mathrm{X} / \mathrm{Fe}]$} & & $N$ & $g \epsilon$ & $\sigma_{121}$ & {$[\mathrm{X} / \mathrm{Fe}]$} & & $N$ & $\log \epsilon$ & $\sigma_{121}$ & {$[\mathrm{X} / \mathrm{Fe}]$} & \\
\hline C I & 8.43 & 6 & 87 & & 0.65 & & 16 & 8.73 & & 1.03 & & 6 & 87 & & 0.79 & 0.13 & 14 & 8.71 & 0.07 & 1.05 & \\
\hline I & & 1 & 49 & (1) & 0.01 & & 2 & 8.71 & & & & 2 & 64 & & & & 2 & 55 & & 0.63 & \\
\hline $\mathrm{Na} \mathrm{I}$ & & 2 & 88 & 1 & 0.17 & & 1 & 6.00 & & & & \begin{tabular}{|l|}
2 \\
\end{tabular} & 20 & & 0.27 & & - & 90 & 0.00 & 0.42 & 09 \\
\hline & & 1 & 20 & 0.20 & 0.13 & & 1 & & & & & 2 & 26 & & -0.03 & & J & 75 & & -0.08 & \\
\hline & & & & & & & & & & & & 2 & 6.37 & & & & 1 & 07 & 0.20 & 0.39 & 23 \\
\hline & & 4 & 23 & 6 & 0.07 & 0 & 5 & 7.04 & & 0.26 & & 5 & .52 & & 0.36 & & 4 & 06 & & .32 & \\
\hline & & 1 & 97 & .20 & 0.06 & & 3 & 6.65 & & 0.25 & & 2 & 69 & & -0.08 & & . & 64 & 02 & .28 & \\
\hline & & 3 & 66 & 0.03 & 0.14 & 0 . & 7 & 5.83 & & 0.2 & & 7 & 6.03 & 9 & -0.0 & & & 81 & & .24 & 08 \\
\hline & & & & & & & 3 & 5.97 & & & & & & & & & 1 & 79 & & 0.22 & \\
\hline & & 6 & & & 00 & & 6 & 2.72 & & & & 4 & & & & & & 75 & & .37 & \\
\hline & & 4 & & & & & & & & & & & & & & & & 50 & & 32 & \\
\hline & & 3 & & & 0.34 & & 2 & .50 & & 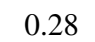 & & 13 & & & & & & 47 & & .29 & \\
\hline & & 3 & & & & & & & & & & 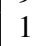 & & & & & & & & & \\
\hline & & & & & & & & & & & & 年 & 3.99 & & & & 2 & 61 & & & \\
\hline & & 4 & & & 000 & & 2 & & & 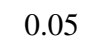 & & . & & & & & & 91 & & & \\
\hline & & & & & & & 2 & & & & & 6 & & & & & 9 & 94 & & 0.06 & \\
\hline & & 1 & 4 & 0.20 & -0.3 & & & & & & & 2 & & & & & (2) & 58 & 20 & -0.08 & \\
\hline & & & & & & & 1 & 5.40 & & & & & & & & & 1 & 60 & 0.20 & -0.06 & \\
\hline & & & & & 0 & & 47 & 6.80 & & & & 43 & 9 & & 0.00 & & 35 & 73 & & 0.00 & \\
\hline & & & 29 & & 0 & & 0 & 6.77 & & 0 & & 17 & 15 & & $0 \Omega 0$ & & 4 & 73 & 0.08 & 0.00 & 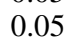 \\
\hline & & 2 & 59 & & & & & & & & & & & & & & & & & & \\
\hline & & $=$ & 78 & & & & 6 & & & & & 1 & & & & & & & & 21 & \\
\hline & & 1 & & & 28 & & 1 & 4.25 & so & 0.76 & & 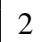 & 92 & & & & & 80 & & 37 & 22 \\
\hline & & 1 & & & -0.2 & & & & & & & 1 & 46 & & 0.25 & & 1 & 06 & 20 & .27 & 29 \\
\hline & & 2 & & & 1.29 & & 4 & 47 & & & & , & 3.25 & & 39 & & 4 & 14 & 08 & .69 & 12 \\
\hline & & 3 & & & & & 2 & 3.61 & & & & 2 & 45 & & 2 & & 2 & 35 & 08 & .54 & 11 \\
\hline & & 6 & & & & & 8 & 282 & & & & 11 & 50 & & & & 6 & 65 & 20 & .32 & 15 \\
\hline & & 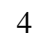 & & & & & & & & & & 5 & & & & & & & & & \\
\hline & & 6 & & & & & & & & & & 1 & & & & & 3 & & & & \\
\hline & & 8 & & & & & & & & & & 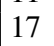 & & & & & & & & & \\
\hline & & 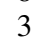 & & & & & & & & & & & & & & & & & & & \\
\hline & & 2 & & & & & 2 & & & & & & & & & & 2 & 39 & & 4 & \\
\hline & & 3 & & & & & 2 & & & & & & & & & & 13 & .99 & 0.12 & 1.69 & \\
\hline & & & & & & & & & & & & & & & & & & & & & \\
\hline & & 2 & & & & & 2 & & & & & 2 & 2.18 & 0.00 & 1.43 & & & & & .80 & \\
\hline & & 4 & & & & & 2 & & & & & & & & & & 3 & 74 & 0.04 & 1.58 & \\
\hline & & & & & & & 1 & & & & & & & & & & 1 & 00 & & 1.93 & \\
\hline & & 2 & & & & & 2 & & & 2.3 & & 1 & 0.85 & 0.20 & 1.10 & 0.2 & 2 & 25 & 20 & 1.92 & 21 \\
\hline & & 2 & 2.19 & 0.09 & 1.55 & 0.16 & 1 & 2.15 & & 2.0 & & & & & & & 2 & 1.85 & 0.20 & 1.77 & .19 \\
\hline W II & 0.85 & & & & & & 1 & 3.07 & 0.20 & 2.95 & 0.25 & & & & & & 1 & 2.30 & 0.20 & 2.21 & 0.25 \\
\hline
\end{tabular}

Notes. For each ion, the table lists the used number of lines $(N)$ for the abundance determination, the determined abundance (log $\epsilon$ ), the uncertainty on this abundance due to line-to-line scatter $\left(\sigma_{121}\right)$, the element over iron ratio $([\mathrm{X} / \mathrm{Fe}])$ and the total uncertainty on $[\mathrm{X} / \mathrm{Fe}]\left(\sigma_{\text {tot }}\right)$ which includes line-to-line scatter and atmospheric parameter uncertainty (see text for details). The solar abundances $\left(\log \epsilon_{\odot}\right)$ in the second column are retrieved from Asplund et al. (2009). For all elemental abundances determined via only one spectral line or via spectrum synthesis, we adopt a line-to-line scatter of 0.20 dex.

The red synthetic spectrum computed using the determined elemental abundances fits the observed spectrum well, except for the strong spectral line at $6006 \AA$. To investigate its identification, we artificially increased the abundances of all elements with spectral lines in this spectral region, but none could produce the observed absorption. This shows that the linelists are not complete for our strongly $s$-process enriched stars, the spectra of which are swamped with transitions of $s$-process elements. Also, for other stars and different spectral regions, we find spectral lines not included in the linelists.

Figure 6 shows a spectrum synthesis check of the determined Nd abundance of IRAS 06530-0213 for the Nd II lines at 6031.270 and $6034.228 \AA$, confirming the high $\mathrm{Nd}$ abundance of IRAS 06530-0213. The spectrum synthesis of the high excitation O I triplet at $6156 \AA$ of IRAS $13245-5036$ is shown in Fig. 7 and shows a good agreement between the observations and determined $\mathrm{O}$ abundances.

We briefly discuss the derived abundances followed by the comparison between our determined results and those of previous studies. In Sect. 5, we specifically discuss the derived $\mathrm{Pb}$ abundances.

\subsection{CNO elements}

For all programme stars, we can determine the carbon and oxygen abundances. The $\mathrm{C} / \mathrm{O}$ ratios range from about 1.0 up to 2.5 
Table 4. Abundance results of IRAS 06530-0213, IRAS 22223+4327, and both observations of IRAS 08143-4406.

\begin{tabular}{|c|c|c|c|c|c|c|c|c|c|c|c|c|c|c|c|c|c|c|c|c|c|}
\hline \multirow[b]{2}{*}{ Ion } & \multirow[b]{2}{*}{$\log \epsilon_{\odot}$} & \multicolumn{5}{|c|}{$\begin{array}{c}\text { IRAS 06530 } \\
{[\mathrm{Fe} / \mathrm{H}]=-0.32} \\
T_{\mathrm{eff}}=7375 \mathrm{~K} \\
\log g=1.25 \mathrm{dex}\end{array}$} & \multicolumn{5}{|c|}{$\begin{array}{c}\text { IRAS 08143 BLUE437 } \\
{[\mathrm{Fe} / \mathrm{H}]=-0.43} \\
T_{\text {eff }}=7000 \mathrm{~K} \\
\log g=1.5 \mathrm{dex}\end{array}$} & \multicolumn{5}{|c|}{$\begin{array}{c}\text { IRAS 08143 RED580 } \\
{[\mathrm{Fe} / \mathrm{H}]=-0.36} \\
T_{\text {eff }}=7250 \mathrm{~K} \\
\log g=1.50 \mathrm{dex}\end{array}$} & \multicolumn{5}{|c|}{$\begin{array}{c}\text { IRAS } 22223 \\
{[\mathrm{Fe} / \mathrm{H}]=-0.30} \\
T_{\text {eff }}=6500 \mathrm{~K} \\
\log g=1.00 \mathrm{dex}\end{array}$} \\
\hline & & $N$ & $\log \epsilon$ & $\sigma_{121}$ & {$[\mathrm{X} / \mathrm{Fe}]$} & tot & $N$ & $\log \epsilon$ & $\sigma_{121}$ & {$[\mathrm{X} / \mathrm{Fe}]$} & $\sigma_{\text {tot }}$ & $N$ & $\log \epsilon$ & $\sigma_{121}$ & {$[\mathrm{X} / \mathrm{Fe}]$} & $\sigma_{\text {tot }}$ & $N$ & $\log \epsilon$ & $\sigma_{121}$ & {$[\mathrm{X} / \mathrm{Fe}]$} & \\
\hline C I & 8.43 & 10 & 8.94 & 0.07 & 0.83 & $\frac{13}{13}$ & 15 & & 0.06 & 0.71 & 0.10 & 11 & 8.74 & 0.09 & 0.67 & 0.12 & 19 & 8.72 & 0.06 & 0.59 & 0.07 \\
\hline N I & 7.83 & & & & & & 7 & 7.41 & & & & & & & & & 2 & 7.68 & 0.30 & 0.15 & 21 \\
\hline O I & 8.69 & 6 & 8.72 & 0.08 & 0.35 & 0.11 & & & & & & 3 & 8.52 & 0.04 & 0.19 & 0.13 & 5 & 8.70 & 0.06 & 0.31 & .13 \\
\hline $\mathrm{Na} \mathrm{I}$ & & 1 & 6.32 & 0.20 & 0.46 & 0.22 & & & & & & 2 & 6.35 & & 0.48 & 0.11 & 2 & 6.20 & & & .08 \\
\hline & & 1 & 7.22 & 0 & -0.0 & & 4 & 7.21 & 0.06 & 0.05 & 0.09 & 2 & 7.22 & 0.04 & -0.01 & 0.10 & 1 & 7.30 & 20 & 0.02 & 22 \\
\hline & ( & & & & & & 2 & 6.40 & 08 & 0.40 & 0.09 & 1 & 6.42 & 0.20 & 0.34 & 0.22 & 2 & 6.10 & 04 & -0.03 & .09 \\
\hline & & 2 & 7.34 & & 0.15 & 0.22 & 4 & 7.54 & 0.06 & 0.46 & 0.17 & 7 & 7.66 & 0.08 & 0.51 & 0.18 & 14 & 7.42 & .10 & 0.21 & 0.17 \\
\hline & & 1 & 7.36 & 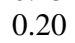 & 0.17 & & & & & & & & & & & & & & & & \\
\hline & & & & & & & 1 & 5.80 & & 2 & & & & & & & & & & & \\
\hline & & 2 & 00 & & 1 & & 7 & 7.1 & & 1 & & 3 & 7.10 & & & 0.15 & 5 & 94 & 8 & 0.12 & 12 \\
\hline & & & 6.18 & & 0.22 & & 3 & 6.1 & & 26 & 0.09 & 12 & 6.16 & & & & 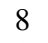 & .01 & 8 & & 07 \\
\hline & & 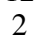 & 08 & & 6 & & & & & & & 4 & 6.11 & & & & 3 & 0 & & & 10 \\
\hline & & & & & & & & & & & & 2 & 3.09 & & & & 4 & 2.95 & & & 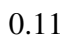 \\
\hline & & & & & & & 5 & 5.03 & 012 & 0.53 & 0.11 & 5 & 5.06 & & & & & & & & \\
\hline & & 5 & .79 & 0.05 & 0.16 & U & & & & & & 5 & 5.01 & & & 0.10 & 5 & 4.54 & & -0.11 & 11 \\
\hline & & & & & & & 2 & 3.7 & & 0.25 & & 1 & 3.74 & & & & 4 & 3.64 & & & 15 \\
\hline & & 2 & & & 0.19 & & 4 & 5.3 & & & & 4 & 5.41 & & & & 6 & 5.22 & & & 09 \\
\hline & & & & & & & 2 & 5.3 & & & & 13 & 5.31 & & & & 11 & 5.29 & & & 0.06 \\
\hline & & 2 & 400 & & -0 & & 3 & 5.0 & & 0.07 & & 2 & 5.05 & & & 0.19 & 2 & 4.98 & & & 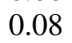 \\
\hline & & & & & & & 1 & 5.0 & & 000 & & 1 & 5.05 & & & 0.23 & & & & & \\
\hline & & & 7.12 & & 000 & & 17 & 7.0 & & 000 & & 70 & 7.13 & & & 0.04 & 80 & 7.18 & & 0.00 & 04 \\
\hline & & 24 & 7 & 0.08 & 00 & 0 & 8 & 7.0 & 0 . & 000 & & 23 & 7.14 & 0. & 0.00 & 0.06 & 25 & 7.20 & & 0.00 & .05 \\
\hline & & & & & & & & & & & & & & & & & 1 & 4.71 & & 0.02 & 0.23 \\
\hline & & 5 & & & 23 & & 13 & 6.0 & 0. & 28 & 0 & 4 & 6.08 & 0.05 & 22 & 0.08 & 13 & 5.87 & 0.07 & -0.03 & 0.06 \\
\hline & & 1 & & & & & & & & & & & & & & & & & & & \\
\hline & & 1 & & & & & & & & & & 1 & & & & & & & & & 22 \\
\hline & & 1 & & & & & & & & & & 1 & 4.60 & & & & 3 & 6 & & & 19 \\
\hline & & 2 & & & & & 3 & 3.4 & & 5 & & 2 & 3.65 & & & & 3 & 24 & & & 11 \\
\hline & & 2 & 3.86 & & 1.60 & U & 5 & 3.85 & 0.1 & 1.70 & 0.12 & 4 & 3.85 & 0.10 & 1.63 & 0.11 & 3 & 3.63 & & & 0.10 \\
\hline & & & & & & & & & & & & & & & & & 1 & 2.89 & & & 0.23 \\
\hline & & & & & & & 1 & & & & & & & & & & 1 & .89 & & & 26 \\
\hline & & & & & & & 1 & & & & & 4 & 2.46 & & & & 4 & & & & 12 \\
\hline & & & & & & & 7 & & & & & & & & & & & & & & 12 \\
\hline & & & & & & & 6 & & & & & 9 & 2.10 & & & & 5 & & & & 15 \\
\hline & & & & & & & 12 & & & & & 9 & 2.67 & & & & 13 & 1.96 & & & 15 \\
\hline & & 2 & & & & & 16 & 1.90 & & 1.51 & & U & 1.91 & & & & $\delta$ & 1.25 & & & .14 \\
\hline & & 2 & & & & & & & & & & 2 & 1.10 & & & & 2 & 0.78 & & & 16 \\
\hline & & 5 & 2.59 & 0.11 & 1.84 & & 8 & 2.00 & 0.06 & .36 & 0.09 & 2 & 2.02 & 0.01 & 1.31 & 0.11 & 1 & 1.67 & 0 & 0.90 & .23 \\
\hline & & & & & & & & & & & & & & & & & 1 & 1.80 & & & 0.24 \\
\hline & & 2 & & & & & 2 & 2.20 & 0.20 & 1.11 & 0.20 & 1 & 2.20 & 0.20 & 1.64 & 0.24 & 1 & 1.18 & 0.20 & 0.56 & 0.23 \\
\hline & & 1 & & & & & & & & & & & & & & & & & & & \\
\hline & & 1 & & & & & 1 & 2.15 & 0.20 & 1.74 & 0.25 & 1 & 2.12 & & & & & & & & \\
\hline & & 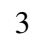 & & & & & & & & & & 1 & 1.00 & 0.20 & .26 & 0.25 & & & & & \\
\hline & & 1 & & & & & & & & & & & & & & & & & & & \\
\hline W II & 0.85 & 1 & 2.98 & 0.20 & 2.45 & 0.25 & & & & & & 1 & 2.15 & 0.20 & 1.00 & 0.25 & 1 & 1.45 & 0.20 & 0.90 & 0.25 \\
\hline
\end{tabular}

Notes. The list of IRAS 08143 BLUE437 contains the results for the Blue437 and Red860 spectra, the list of IRAS08143 RED580 shows the results for the Red580 spectra (see Sect. 2). Same as for Table 3.

and are listed in the second column of Table 8 . The errors on the $\mathrm{C} / \mathrm{O}$ ratios in Table 8 include line-to-line scatter and atmospheric model uncertainties. They can become significant because of the strong temperature sensitivity of the available $\mathrm{C}$ and $\mathrm{O}$ lines within the temperature range of the sample stars. The two new post-AGB stars, IRAS 13245-5036 and IRAS 14429-4539, display $\mathrm{C} / \mathrm{O}$ ratios of about 1.1 and 1.3 , respectively. This also confirms the observation that the $21 \mu \mathrm{m}$ feature is only detected around carbon-rich post-AGB stars.

For six of the stars, we could determine nitrogen abundances using non-LTE sensitive spectral lines between 7000 and $9000 \AA$ A. We applied non-LTE corrections to the LTE N abundances following the temperature-dependent corrections of Fig. 4 in Lyubimkov et al. (2011), which shows a larger correction for higher temperatures. We adopted the following corrections: for IRAS $22223+4327$, we use a correction of -0.3 dex; for IRAS $08143-4406$, we apply a correction of $-0.4 \mathrm{dex}$; for IRAS $07143+1005$ and IRAS 17279-1119, we apply corrections of $-0.5 \mathrm{dex}$; and for IRAS 14325-6428 and IRAS 19500-1709, we use corrections of $-0.65 \mathrm{dex}$ and $-0.7 \mathrm{dex}$, respectively. These corrections are already included in the listed results of Tables 4-6. Given the uncertainties on the non-LTE correction, 
K. De Smedt et al.: Pb in Galactic $s$-process enriched post-AGB stars

Table 5. Abundance results of IRAS 07134+1005, IRAS 08281-4850, IRAS 17279-1119, and IRAS 19500-1709.

\begin{tabular}{|c|c|c|c|c|c|c|c|c|c|c|c|c|c|c|c|c|c|c|c|c|c|}
\hline \multirow[b]{2}{*}{ Ion } & \multirow[b]{2}{*}{$\log \epsilon_{\odot}$} & \multicolumn{5}{|c|}{$\begin{array}{c}\text { IRAS 07134 } \\
{[\mathrm{Fe} / \mathrm{H}]=-0.91} \\
T_{\text {eff }}=7250 \mathrm{~K} \\
\log g=0.5 \mathrm{dex}\end{array}$} & \multicolumn{5}{|c|}{$\begin{array}{c}\text { IRAS 08281 } \\
{[\mathrm{Fe} / \mathrm{H}]=-0.26} \\
T_{\mathrm{eff}}=7875 \mathrm{~K} \\
\log g=1.25 \mathrm{dex}\end{array}$} & \multicolumn{5}{|c|}{$\begin{array}{c}\text { IRAS } 17279 \\
{[\mathrm{Fe} / \mathrm{H}]=-0.64} \\
T_{\text {eff }}=7250 \mathrm{~K} \\
\log g=1.25 \mathrm{dex}\end{array}$} & \multicolumn{5}{|c|}{$\begin{array}{c}\text { IRAS } 19500 \\
{[\mathrm{Fe} / \mathrm{H}]=-0.59} \\
T_{\text {eff }}=8000 \mathrm{~K} \\
\log g=1.00 \mathrm{dex}\end{array}$} \\
\hline & & $N$ & $\log \epsilon$ & $\sigma_{121}$ & {$[\mathrm{X} / \mathrm{Fe}]$} & tot & $N$ & $\log \epsilon$ & $\sigma_{121}$ & {$[\mathrm{X} / \mathrm{Fe}]$} & $\sigma_{\text {tot }}$ & $N$ & $\log \epsilon$ & $\sigma_{121}$ & {$[\mathrm{X} / \mathrm{Fe}]$} & $\sigma_{\text {tot }}$ & $N$ & $\log \epsilon$ & $\sigma_{121}$ & {$[\mathrm{X} / \mathrm{Fe}]$} & $\sigma_{\text {tot }}$ \\
\hline C I & 8.43 & 47 & 8.68 & 0.08 & 1.16 & 0.22 & 16 & 8.91 & 0.05 & 0.75 & 0.21 & 17 & 8.36 & 0.09 & 0.47 & 0.14 & 15 & 8.82 & 0.06 & 0.99 & 0.33 \\
\hline N I & 7.83 & 3 & 7.50 & 30 & 0.57 & 0.19 & & & & & & 1 & 7.27 & & & 0.35 & 3 & 7.65 & & 41 & 17 \\
\hline O I & 69 & 9 & 8.59 & & 81 & 0.19 & 5 & 8.54 & 0 & 0.12 & 0.11 & 2 & 8.39 & & 0.24 & 16 & 8 & 3.81 & & 0.72 & .09 \\
\hline $\mathrm{Na} \mathrm{I}$ & 4 & & & & & & 1 & 6.65 & & 0.70 & & 2 & 6.25 & & 52 & & & & & & \\
\hline I & DU & 4 & 689 & & 026 & & 3 & 7.38 & & 0.07 & & 2 & 7.25 & 6 & 6 & 2 & 2 & 31 & & 0.29 & 0.07 \\
\hline g II & 60 & & & & & & & & & & & & & & & & 3 & 7.29 & & 0.28 & 0.10 \\
\hline & .45 & & & & & & & & & & & & & & & & 1 & 5.51 & & -0.36 & 0.21 \\
\hline & 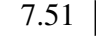 & & & & & & 2 & 750 & 020 & 0.25 & 031 & 6 & 7.33 & 0.10 & 0.36 & 0.20 & 1 & 7.03 & & 0.11 & .49 \\
\hline II & 751 & & & & & & & & & & & 1 & 7.33 & 0.20 & 36 & 0.26 & 2 & 7.01 & & 0.10 & .13 \\
\hline & 7 & 1 & 6.59 & 0.20 & 0.38 & 0.35 & 3 & 7.24 & 0.03 & 0.38 & 0.24 & 2 & 6.91 & 0.02 & 0.33 & 0.18 & & & & & \\
\hline & 5 & 2 & 4.68 & 007 & 0.61 & 0.12 & & & & & & & & & & & & & & & \\
\hline & 34 & 11 & 5.44 & 0.05 & 0.07 & 0 & 5 & 6.29 & 0. & 0.25 & 0 & 7 & 6.00 & 0.07 & 17 & 0.09 & 3 & 6.08 & 6 & 0.32 & 0.23 \\
\hline & 6 & 3 & 5.47 & 0.03 & 0.04 & 0 & 2 & 6.18 & 0. & 0.10 & 0. & 1 & 6.00 & 0.20 & 20 & 0.25 & 3 & 5.92 & 8 & 0.18 & 0.26 \\
\hline & 3.15 & 8 & 2.41 & 0.06 & 0.17 & 0.09 & 5 & 3.19 & 0.04 & 0.30 & 0.13 & 7 & 2.71 & 0.09 & 10 & 0.12 & 8 & 2.78 & 0.11 & 0.23 & 0.22 \\
\hline & 495 & & & & & & & & & & & 3 & 4.62 & 0.06 & 0.18 & 0.12 & & & & & \\
\hline II & 4 & 24 & 4.18 & 0.09 & 0.14 & 0.08 & 12 & 4.74 & 0.04 & 0.05 & 0.09 & 16 & 4.65 & 0.13 & .24 & 0.10 & 33 & 4.54 & .09 & 0.19 & 0.14 \\
\hline II & 2 & 5 & 3.32 & & 30 & & 1 & 3.68 & & 0.02 & & 5 & 3.92 & & & 0.13 & 11 & 3.62 & & & .12 \\
\hline & 5 & 3 & 4.70 & & & 6 & 1 & 5.40 & 0. & 0.05 & 0 & 2 & 5.17 & 0 & 4 & 1 & 3 & 5.12 & & & 11 \\
\hline & & 14 & 5 & & & & 12 & 5.33 & 0. & -0.05 & 0.06 & 13 & 5.14 & 0.11 & .04 & 0.10 & 24 & 5.14 & & & 07 \\
\hline & & 2 & & & & & & & & & & & & & & & 2 & 4.75 & & & 99 \\
\hline & & 4 & & & & & & & & & & 2 & & & & & 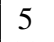 & & & & 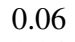 \\
\hline & & & 3 & & & & 44 & 721 & & $0 \cap 0$ & 0.0 & 44 & 6.99 & & & 0.06 & 33 & 6.92 & & & \\
\hline & & 32 & 6.59 & 0.0 & .00 & 0 & 23 & 7.24 & 0.0 & 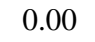 & 0.05 & 30 & 6.96 & 0 . & 0 & 0.08 & 32 & 6.91 & 0. & 0.00 & 0. \\
\hline C & 4.96 & & & & & & & & & & & 2 & 4.28 & 0.14 & -0.2 & 0.17 & & & & & \\
\hline & & & & & & & & & & & & & & & & & 1 & 0 & & & 0.22 \\
\hline & & 5 & & & & & & & & & & 4 & & & & & 3 & & & & 08 \\
\hline $\mathrm{Ni}$ & 0.22 & 1 & 5.32 & 0.20 & ren & 0.26 & 2 & 5.90 & 0.20 & -0.06 & 0.19 & 4 & 6.07 & 0.07 & 0.39 & 0.11 & 2 & 5.80 & 0.07 & 0.18 & 0.09 \\
\hline $\mathrm{Zn} \mathrm{I}$ & & & & & & & & & & & & 1 & 4.25 & 0.20 & 0.23 & 0.31 & & & & & \\
\hline Y II & & 12 & 2.97 & & 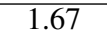 & & 7 & 3.66 & & 1.72 & 0.19 & 10 & 2.38 & 0.12 & 0.71 & 0.12 & 11 & 3.02 & & 40 & 0.29 \\
\hline & & 15 & 3.28 & & & & 12 & 3.74 & & & 0.11 & 10 & 2.82 & 0.11 & 0.77 & 0.12 & 22 & 3.32 & & & 0.19 \\
\hline Mo II & & 1 & 2.68 & & & & 1 & 3.35 & & 1.73 & 0.23 & & & & & & 2 & 2.88 & 8 & & 0.12 \\
\hline Ba II & 2.18 & 2 & 3.09 & 0.02 & 1.82 & 0.30 & 1 & 3.80 & 0.20 & 1.88 & 0.36 & 1 & 2.80 & 0.20 & 1.16 & 0.31 & 1 & 3.00 & 0.20 & 1.42 & 0.49 \\
\hline La II & 1.1 & 29 & 2.04 & 0.0 & 185 & 0.24 & 17 & 2.70 & & 1.87 & & 7 & 1.48 & & & 0.17 & 5 & 1.87 & 9 & & 0.43 \\
\hline Ce II & 1.5 & 41 & 2.27 & & 150 & 0 & 21 & 2.85 & & & 0.24 & 11 & 2.02 & 0.10 & & 0.15 & 7 & 2.28 & 0.12 & 1.29 & 0.39 \\
\hline Pr II & 0.72 & 5 & 1.43 & & & & 3 & 2.40 & & 1.94 & & 5 & 1.35 & & & 0.21 & & & & & \\
\hline Nd II & 1.42 & 29 & 2.14 & & & 0.29 & 15 & 2.83 & 0.09 & 1.67 & 0.30 & 8 & 1.64 & 0.07 & 0.75 & 0.19 & 5 & 2.31 & 0.03 & 1.48 & 0.44 \\
\hline Sm II & 0.9 & 13 & 1.49 & & & & & & & & & 3 & 1.46 & 0.08 & 1.03 & 0.19 & & & & & \\
\hline Eu II & 0.5 & 2 & 0.20 & 020 & & & 2 & 1.00 & ( & 0.74 & & & & & & & 1 & .10 & 20 & 8 & .48 \\
\hline Gd II & 1.0 & 6 & 1.33 & & & & 2 & 1.98 & 0.02 & 1.18 & 0.18 & & & & & & 2 & 1.52 & 0.18 & 1.05 & 0.34 \\
\hline Dy II & & 2 & 1.14 & 0.2 & & & 1 & 1.90 & & & & & & & & & & & & & \\
\hline & 0.9 & 0 & 1.41 & 0.0 & & & 1 & 2.19 & 0. & & & & & & & & & & & & \\
\hline Lu II & 0.1 & 4 & 0.80 & 0.20 & 1.61 & 0.22 & 1 & 1.3 & 0. & 1. & & 1 & & & & 0. & 2 & & & & 0.21 \\
\hline Hf II & 0.85 & & & & & & 2 & 2.35 & 0.20 & 1.76 & 0.2 & 1 & 1.04 & 0.20 & 0 & 0.25 & 1 & 1.53 & 0 & 1.27 & 0.25 \\
\hline
\end{tabular}

Notes. Same as for Table 3.

we assume a somewhat ad-hoc, line-to-line scatter of $0.3 \mathrm{dex}$ for all $\mathrm{N}$ abundance determinations.

\subsection{S-process elements}

The two new post-AGB stars, IRAS 13245-5036 and IRAS 14429-4539, show strong $s$-process enrichments with a stronger overabundance of the hs elements with respect to the ls elements. Both stars are warm and, hence, intrinsically display a less rich spectrum. We could only determine a limited set of $s$-process elements for IRAS 14429-4539. The $s$-process enrichments confirm the evolved nature of both stars. This corroborates the finding that the stars displaying the $21 \mu \mathrm{m}$ feature are all $s$-process rich and carbon-rich post-AGB stars, which are the likely descendants of AGB carbon stars.

We confirm that all other programme stars show $s$-process enhancements for both the ls and hs elements with significant differences between the objects. IRAS 17279-1119 displays a mild $s$-process enrichment and most of the $s$-process elements have an $[\mathrm{X} / \mathrm{Fe}]<1.0 \mathrm{dex}$, while the two strongest enriched objects, IRAS 05341+0852 and IRAS 06530-0213, have high $s$-process $[\mathrm{X} / \mathrm{Fe}]$ abundances reaching up to $2.5 \mathrm{dex}$. The abundances derived from the two observational settings are similar for IRAS 08143-4406 (upper right panel of Fig. 9).

Apart from the different levels of enrichment, we also find different abundance distributions. For IRAS 08143-4406, IRAS 14325-6428, IRAS 19500-1709, and IRAS 22223+4327, 
Table 6. Abundance results of IRAS 13245-5036, IRAS 14325-6428, and IRAS 14429-4539.

\begin{tabular}{|c|c|c|c|c|c|c|c|c|c|c|c|c|c|c|c|c|}
\hline \multirow[b]{2}{*}{ Ion } & \multirow[b]{2}{*}{$\log \epsilon_{\odot}$} & \multicolumn{5}{|c|}{$\begin{array}{c}\text { IRAS } 13245 \\
{[\mathrm{Fe} / \mathrm{H}]=-0.30} \\
T_{\mathrm{eff}}=9500 \mathrm{~K} \\
\log g=2.75 \mathrm{dex}\end{array}$} & \multicolumn{5}{|c|}{$\begin{array}{c}\text { IRAS } 14325 \\
{[\mathrm{Fe} / \mathrm{H}]=-0.56} \\
T_{\text {eff }}=8000 \mathrm{~K} \\
\log g=1.00 \mathrm{dex}\end{array}$} & \multicolumn{5}{|c|}{$\begin{array}{c}\text { IRAS } 14429 \\
{[\mathrm{Fe} / \mathrm{H}]=-0.18} \\
T_{\text {eff }}=9375 \mathrm{~K} \\
\log g=2.50 \mathrm{dex}\end{array}$} \\
\hline & & $N$ & $\log \epsilon$ & $\sigma_{121}$ & {$[\mathrm{X} / \mathrm{Fe}]$} & $\sigma_{\text {tot }}$ & $N$ & $\log \epsilon$ & $\sigma_{121}$ & {$[\mathrm{X} / \mathrm{Fe}]$} & $\sigma_{\text {tot }}$ & $N$ & $\log \epsilon$ & $\sigma_{121}$ & {$[\mathrm{X} / \mathrm{Fe}]$} & $\sigma_{\text {tot }}$ \\
\hline C I & 8.43 & 4 & 8.70 & 0.00 & 0.57 & 0.21 & 24 & 9.06 & 0.10 & 1.18 & 0.23 & 3 & 8.93 & 0.08 & 0.68 & 023 \\
\hline N I & 7.83 & & & & & & 7 & 7.45 & 0.30 & 0.18 & 0.20 & & & & & \\
\hline O I & 8.69 & 5 & 8.65 & 0.12 & 0.26 & 0.13 & 10 & 8.70 & 0.06 & 0.57 & 0.09 & 6 & 8.82 & 0.04 & 0.31 & 0.12 \\
\hline $\mathrm{Na} \mathrm{I}$ & 6.24 & & & & & & 1 & 6.50 & 0.20 & 0.81 & 0.22 & & & & & \\
\hline $\mathrm{Mg} \mathrm{I}$ & 7.60 & 5 & 7.16 & 0.07 & -0.09 & 0.09 & 4 & 7.51 & 0.05 & 0.47 & 0.08 & 3 & 7.32 & 0.04 & -0.01 & 0.09 \\
\hline Mg II & 7.60 & 2 & 7.20 & 0.07 & -0.11 & 0.11 & 5 & 7.37 & 0.13 & 0.33 & 0.14 & 3 & 7.29 & 0.03 & -0.13 & 0.10 \\
\hline $\mathrm{Al} \mathrm{I}$ & 6.45 & 1 & 5.75 & 0.20 & -0.35 & 0.23 & 1 & 5.70 & 0.20 & -0.2 & 0.21 & 2 & 5.89 & 0.01 & -0.3 & 0.09 \\
\hline Al II & 6.45 & & & & & & & & & & & 1 & 5.97 & 0.20 & -0.3 & 0.26 \\
\hline Si I & 7.51 & 1 & 7.19 & 0.20 & -0.02 & 0.33 & 1 & 6.85 & 0.20 & -0.1 & 0.38 & 1 & 7.10 & 0.20 & -0.23 & 0.33 \\
\hline Si II & 7.51 & 3 & 7.19 & 0.07 & -0.02 & 0.12 & 2 & 6.83 & 0.00 & -0.12 & 0.18 & 1 & 7.15 & 0.20 & -0.18 & 0.25 \\
\hline S I & 7.12 & & & & & & 1 & 7.20 & 0.20 & 0.64 & 0.32 & & & & & \\
\hline I & 6.34 & & & & & & 5 & 6.23 & 0.10 & 0.44 & 0 & & & & & \\
\hline Ca II & 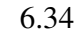 & & & & & & 2 & 6.26 & 0.03 & 0.48 & 0.20 & & & & & \\
\hline Sc II & 5 & 6 & 3.29 & 0.11 & 0.44 & 0.18 & 1 & 2.97 & 0.11 & 0.38 & 0.19 & 8 & .38 & 2 & 0.41 & 0.20 \\
\hline $\mathrm{Ti}$ & 5 & 22 & 4.96 & 0 & 0.31 & 0.12 & 8 & 4.57 & & & & 42 & .03 & & 0.26 & \\
\hline V II & 3 & 2 & 3.73 & 0.03 & 0.10 & 0.11 & 11 & 3.60 & 0.09 & 0 & 2 & 4 & 3.99 & & 0.24 & 0 \\
\hline $\mathrm{Cr}$ & 5.6 & & & & & & 3 & 5.28 & 0.09 & 0.19 & & & & & & \\
\hline $\mathrm{Cr}$ & 5 & 11 & 5.44 & 0.07 & 0.10 & 0.07 & 22 & 5.19 & 0.07 & 0.11 & 0.05 & 28 & 5.51 & 0.0 & 0.05 & 0.07 \\
\hline Mn I & 5.43 & & & & & & 1 & 4.94 & 0.20 & 0.06 & 0.23 & & & & & \\
\hline Mn II & 5 & & & & & & & & & & & 2 & 5.30 & 0.0 & 0.05 & 0.10 \\
\hline $\mathrm{Fe} \mathrm{I}$ & 7 & 21 & 7.15 & 0.10 & 0.00 & 0.07 & 26 & 6.95 & 0.06 & 0.00 & 0.04 & 26 & 7.24 & 0. & 0.00 & 0.06 \\
\hline $\mathrm{Fe}$ II & 7.50 & 41 & 7.20 & 0.06 & 0.00 & 0.04 & 58 & 6.94 & 0.06 & 0.00 & 0.04 & 52 & 7.32 & 0.09 & 0.00 & 0.05 \\
\hline Ni I & 6. & & & & & & 3 & 5.78 & 0.06 & 0.12 & 0.08 & & & & & \\
\hline Ni II & 6 & 4 & 5.93 & 0.07 & 0.01 & 0.09 & 4 & 5.74 & 0.10 & 0.08 & 0.10 & 4 & 5.97 & 0.10 & -0.07 & 0.11 \\
\hline Y II & 2 & 11 & 3.31 & 0.0 & 1.40 & 0.23 & 10 & 2.99 & 0.12 & 1.34 & 0.26 & 10 & 3.15 & 0.1 & 1.13 & 0.25 \\
\hline $\mathrm{Zr}$ II & 2 & 17 & 4.00 & 0.0 & 1.72 & 0.15 & 17 & 3.18 & 0.12 & 1.16 & 0.16 & 13 & 3.86 & 0. & 1.46 & 0.17 \\
\hline Ba II & 2 & 1 & 4.00 & 0.20 & 2.12 & 0.34 & 1 & 3.10 & 0.20 & 1.48 & 0.42 & & & & & \\
\hline La II & 1 & 3 & 3.02 & 0.1 & 2 & 0.28 & 7 & 1.85 & 0.06 & 1.31 & 0.35 & 4 & 2.61 & 0.11 & 1.69 & 0.29 \\
\hline Ce I & 1 & 4 & 3.40 & 0.2 & 2 & 0.28 & 3 & 2.29 & 0.20 & 1.27 & 0.35 & & & & & \\
\hline Pr III & 0. & 2 & 2.83 & 0.0 & 2.41 & 0.11 & & & & & & & & & & \\
\hline $\mathrm{Nd}$ & 1 & & & & & & 2 & 2.25 & 0.20 & 1.39 & 0.40 & & & & & \\
\hline Nd III & 1.42 & 12 & 3.30 & 0.0 & 2.18 & . & & & & & & 15 & 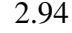 & 09 & 1.70 & 0.00 \\
\hline Sm II & 0.96 & & & & & & 1 & 1.75 & 0.20 & 35 & 0.41 & & & & & \\
\hline Eu II & 0.52 & & & & & & 1 & 0.75 & 0.20 & 0.79 & 0.41 & & & & & \\
\hline Gd II & 1.07 & & & & & & 1 & 1.76 & 0.20 & 1.25 & 0.3 & & & & & \\
\hline Dy II & 1.10 & & & & & & 1 & 1.48 & 0.20 & 0.94 & 0.39 & & & & & \\
\hline Dy III & 1.10 & 1 & 2.79 & 0.20 & 1.99 & 0.23 & & & & & & & & & & \\
\hline & & & & & & & 1 & 0.85 & 0.20 & 1.3 & 0.28 & & & & & \\
\hline Hf II & 0.85 & & & & & & 1 & 1.53 & 0.20 & 1.24 & 0.25 & 1 & 2.54 & 0.2 & 1.87 & 0.25 \\
\hline
\end{tabular}

Notes. Same as for Table 3 .

the enrichment of the ls elements $\mathrm{Y}$ and $\mathrm{Zr}$ is higher or similar to that of the hs elements, while for the other stars the situation is reversed. We go into more detail about the hs/ls behaviour in Sect. 6.

For those objects with Mo determination, we find that Mo has similar enhancements as $\mathrm{Y}$ and $\mathrm{Zr}$. The elements with $Z>62$ are also enriched and their abundance distributions strongly vary from star to star to a level comparable to or less than that of the hs elements.

\subsection{Comparison with previous studies}

In Appendix B, we provide the comparison between our abundance results and those of previous abundance studies (see second to last column of Table 1). For IRAS 13245-5036 and IRAS 14429-4539, there are no available data in literature. We remark that previous studies used different linelists and solar abundances, which can explain small differences between our determinations and previous determinations. Especially, changes in oscillator strength $\log g f$ can significantly impact the individual spectral line abundances.

\section{5. $\mathrm{Pb}$ abundance determination}

For each star, we use an artificially high abundance to theoretically predict which optical $\mathrm{Pb}$ line $(\mathrm{Pb} \mathrm{I}$ or $\mathrm{Pb} \mathrm{II})$ is the strongest for a specific set of atmospheric parameters. We then select the $\mathrm{Pb}$ line that gives the largest predicted EW. For some stars, both $\mathrm{Pb}$ lines result in a similar theoretical $\mathrm{EW}$, so we can use both lines to constrain the $\mathrm{Pb}$ abundance.

Depending on the effective temperature of the sample stars, the $\mathrm{Pb}$ abundances are probed either using the strongest $\mathrm{Pb}$ I line at $4057.807 \AA$ or $\mathrm{Pb}$ II line at $5608.853 \AA$. As the $\mathrm{Pb}$ I line lies in a low $\mathrm{S} / \mathrm{N}$ region with many spectral blends and the $\mathrm{Pb}$ II line is generally very small so that it can be confused with noise in the spectrum, none of the sample stars display a clear $\mathrm{Pb}$ line feature 
K. De Smedt et al.: Pb in Galactic s-process enriched post-AGB stars

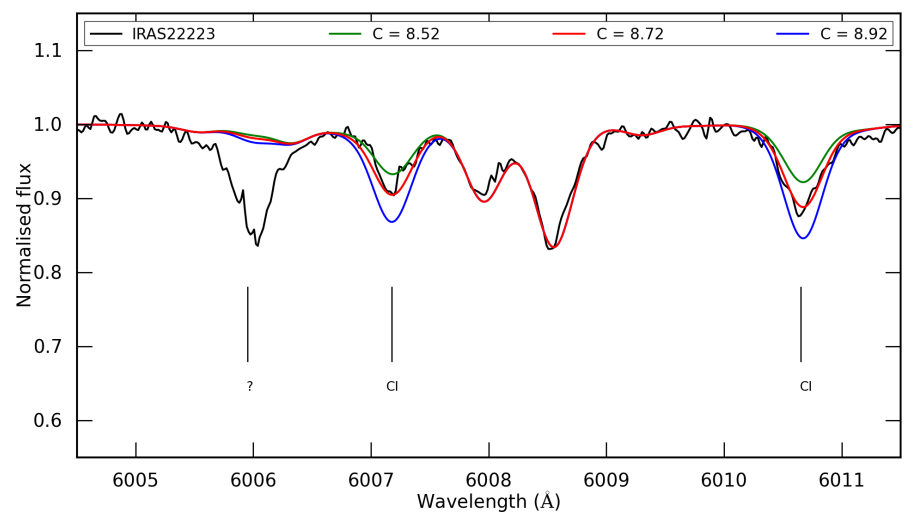

Fig. 5. Spectrum synthesis of the C I lines at 6007.176 and $6010.675 \AA$ for IRAS $22223+4327$. The black spectrum is the observed HERMES spectrum; the coloured spectra represent synthetic spectra with different $\mathrm{C}$ abundances. The red line represents the determined $\mathrm{C}$ abundance; the green and blue spectra represent synthetic spectra with a $\mathrm{C}$ abundance of -0.2 dex and +0.2 dex, respectively.

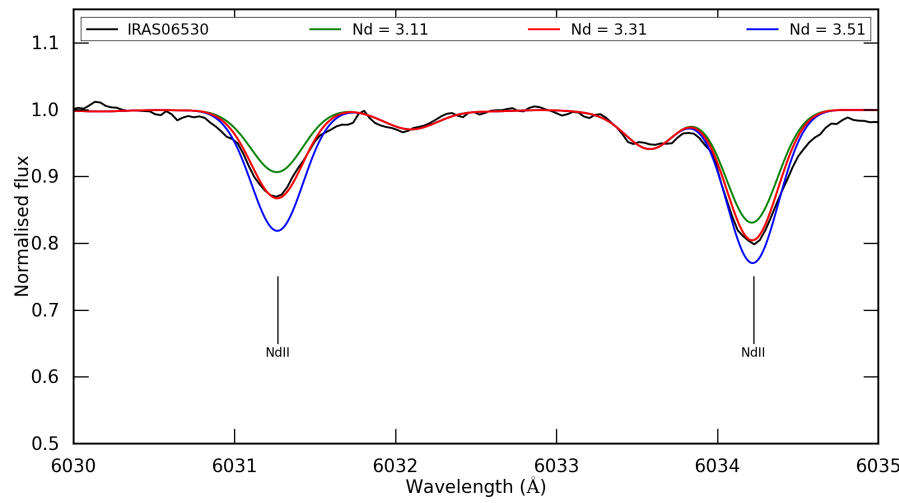

Fig. 6. Spectrum synthesis of the Nd II lines at 6031.270 and 6034.228 A for IRAS 06530-0213. The black spectrum is the observed UVES spectrum; the coloured spectra represent synthetic spectra with different $\mathrm{Nd}$ abundances. The red line represents the determined $\mathrm{Nd}$ abundance; the green and blue spectra represent synthetic spectra with a $\mathrm{Nd}$ abundance of -0.2 dex and +0.2 dex, respectively.

from which an accurate $\mathrm{Pb}$ abundance can be derived. Therefore, we can only determine upper limits on the $\mathrm{Pb}$ abundance.

We generate synthetic spectra for the spectral region of the $\mathrm{Pb}$ line, including all the derived abundances; these are listed in Tables 3 to 6 . We use the synthetic spectral lines to estimate the local position of the continuum at the $\mathrm{Pb}$ line. The lowest $\mathrm{Pb}$ abundance for which the synthetic spectrum fully includes the observed spectral blend at the $\mathrm{Pb}$ line is adopted as upper limit of the $\mathrm{Pb}$ abundance. Examples of our method are illustrated in Figs. 11-13 for three different sample stars.

Figure 11 shows the $\mathrm{Pb}$ abundance determination of IRAS $22223+4327$ using the Pb I line at $4057.807 \AA$. For stars with lower temperatures and/or high metallicities, this spectral region contains many spectral blends. The $\mathrm{S} / \mathrm{N}$ in this blue region is generally only between 20 and 30 . We use the identified spectral blends at $4057.5 \AA$ and $4058.9 \AA$ to estimate the position of the continuum and then use different synthetic $\mathrm{Pb}$ abundances to determine the upper limit. For each figure, the red synthetic spectra represent our adopted abundance upper limit and the dashed black lines show synthetic spectra without any $\mathrm{Pb}$ contribution included.

In case of IRAS $22223+4327$, there is no clear contribution from $\mathrm{Pb}$. The blue synthetic spectrum in Fig. 11 provides a full

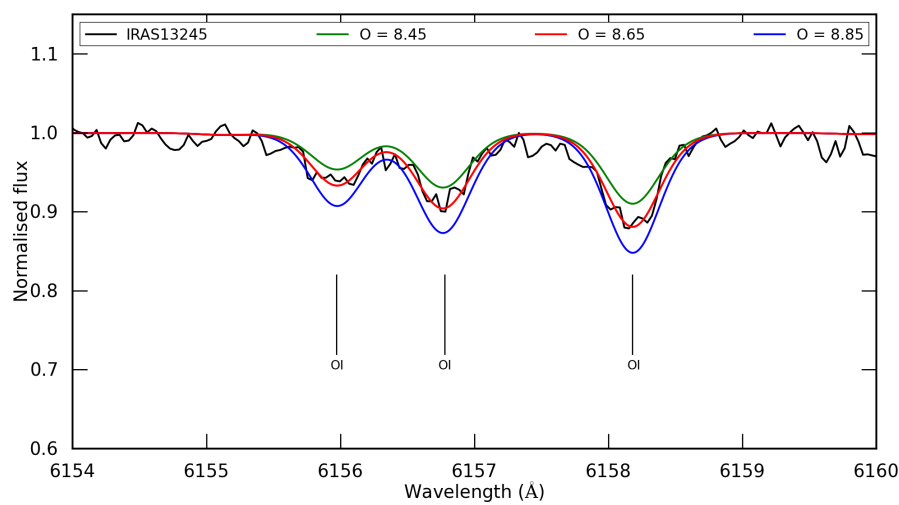

Fig. 7. Spectrum synthesis of the O I triplet at $6156 \AA$ for IRAS 13245 5036. The black spectrum is the observed UVES spectrum, the coloured spectra represent synthetic spectra with different $\mathrm{O}$ abundances. The red line represents the determined $\mathrm{O}$ abundance; the green and blue spectra represent synthetic spectra with an $\mathrm{O}$ abundance of -0.2 dex and +0.2 dex, respectively.

observed feature around $4057.807 \AA$, but generates a large contribution to the blend with the line at $4057.5 \AA$ that is not observed in the spectrum. Therefore, we adopt an upper limit of the $\mathrm{Pb}$ abundance for IRAS 22223+4327, which only creates a contribution to the blend that is still compliant with the observed spectrum. Figure 11 also shows that there are a number of spectral lines in this region that are not included in the linelists. The line features at $4056.2 \AA$ and $4060.5 \AA$ are examples. Moreover, for stars with $T_{\text {eff }}<7500 \mathrm{~K}$ and $[\mathrm{Fe} / \mathrm{H}]>0.5 \mathrm{dex}$, we cannot reproduce the blended line at $4058.2 \mathrm{~A}$ at the red side of the $\mathrm{Pb} \mathrm{I}$ line, which would otherwise be a major factor for constraining the local continuum position.

The $\mathrm{Pb}$ abundance determinations of IRAS 06530-0213 are presented in Fig. 12. The upper panel shows the determination via the $\mathrm{Pb}$ I line at $4057.807 \AA$; the lower panel shows the same for the $\mathrm{Pb}$ II line at $5608.853 \AA$. Also, for this star, we do not detect a clear $\mathrm{Pb}$ I line and we use the spectral blends at 4057.5 and $4058.9 \AA$ to estimate the position of the continuum. We adopt a $\mathrm{Pb}$ abundance that generates a synthetic $\mathrm{Pb}$ line, which fully incorporates the observed line feature at $4057.807 \AA$. For the $\mathrm{Pb}$ II line at $5608.853 \AA$, we use the continuum between 5606.3 and $5608 \AA$ in combination with the blend at $5610.3 \AA$ to estimate the position of the continuum at $5608.853 \AA$. The $\mathrm{Pb}$ II line lies in a spectral region where the continuum is affected by a blend of small unidentified spectral lines, ranging from 5608 up to $5610 \AA$ A. The observed spectrum lies below the continuum of the synthetic spectra. To make sure this feature is real, we checked the individual observed spectra of IRAS 065300213 in Table 1 and found the feature to be present in both observations. We also compared our new spectra with the old observations from Reyniers et al. (2004) and this broad feature is present there as well. We therefore expect this depression to be real. This is likely a set of unresolved small lines. For the $\mathrm{Pb}$ II line, we adopt a $\mathrm{Pb}$ abundance that fits the observed spectrum. However, comparison with the spectra of Reyniers et al. (2004) suggest that the small line at $5608.853 \AA$ in the broad feature between 5608 and $5610 \AA$ is not significant as it is not seen in the older spectra. As a result of this possibility, in combination with the lower upper limit found for the $\mathrm{Pb} I$ line, we adopt the limit for the $\mathrm{Pb} \mathrm{I}$ line as the upper limit of the $\mathrm{Pb}$ abundance for IRAS 06530-0213. 


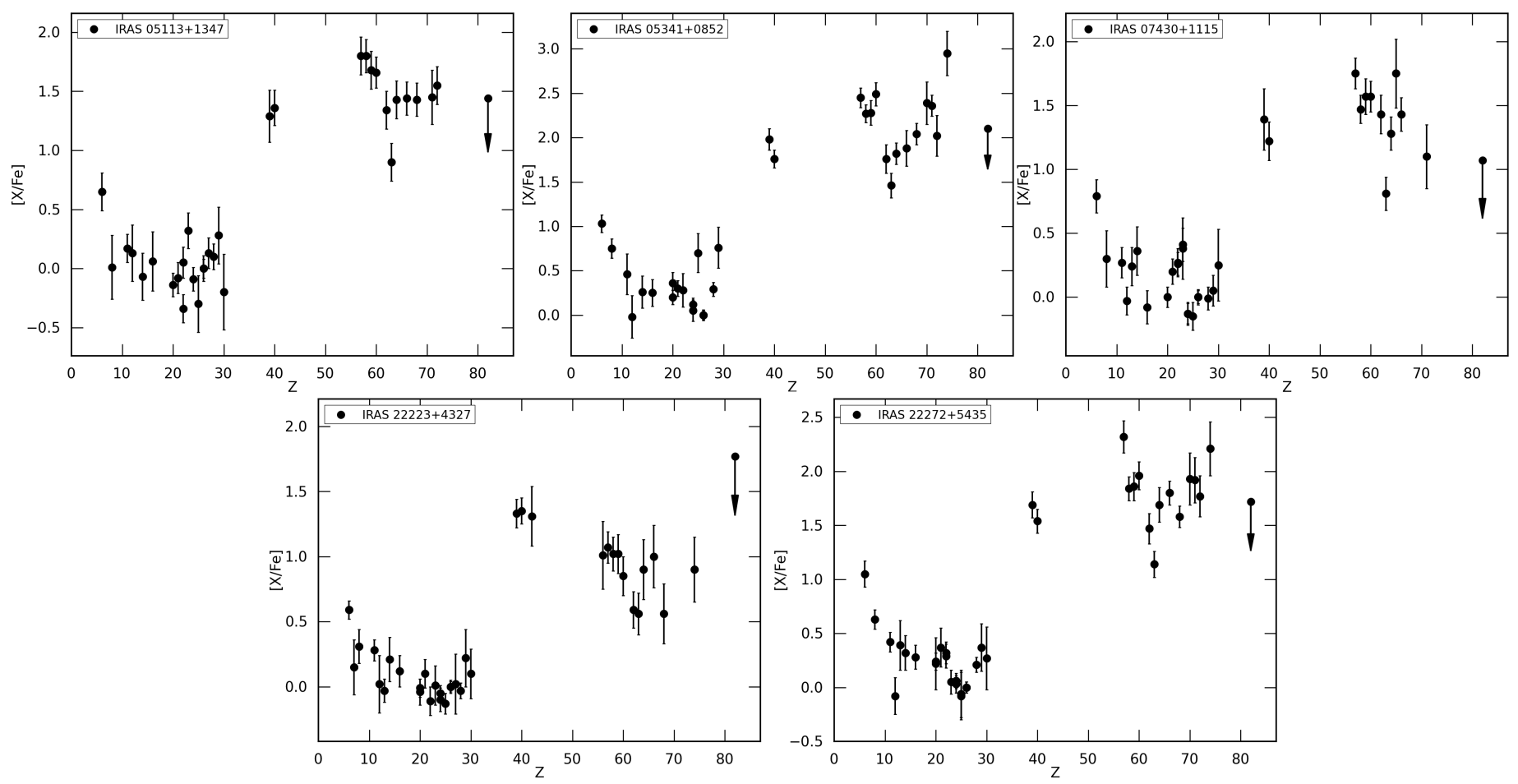

Fig. 8. Element-over-iron ratios for the programme stars with $T_{\text {eff }}<7000 \mathrm{~K}$. The down arrow at $Z=82$ displays the adopted Pb abundance upper limits.

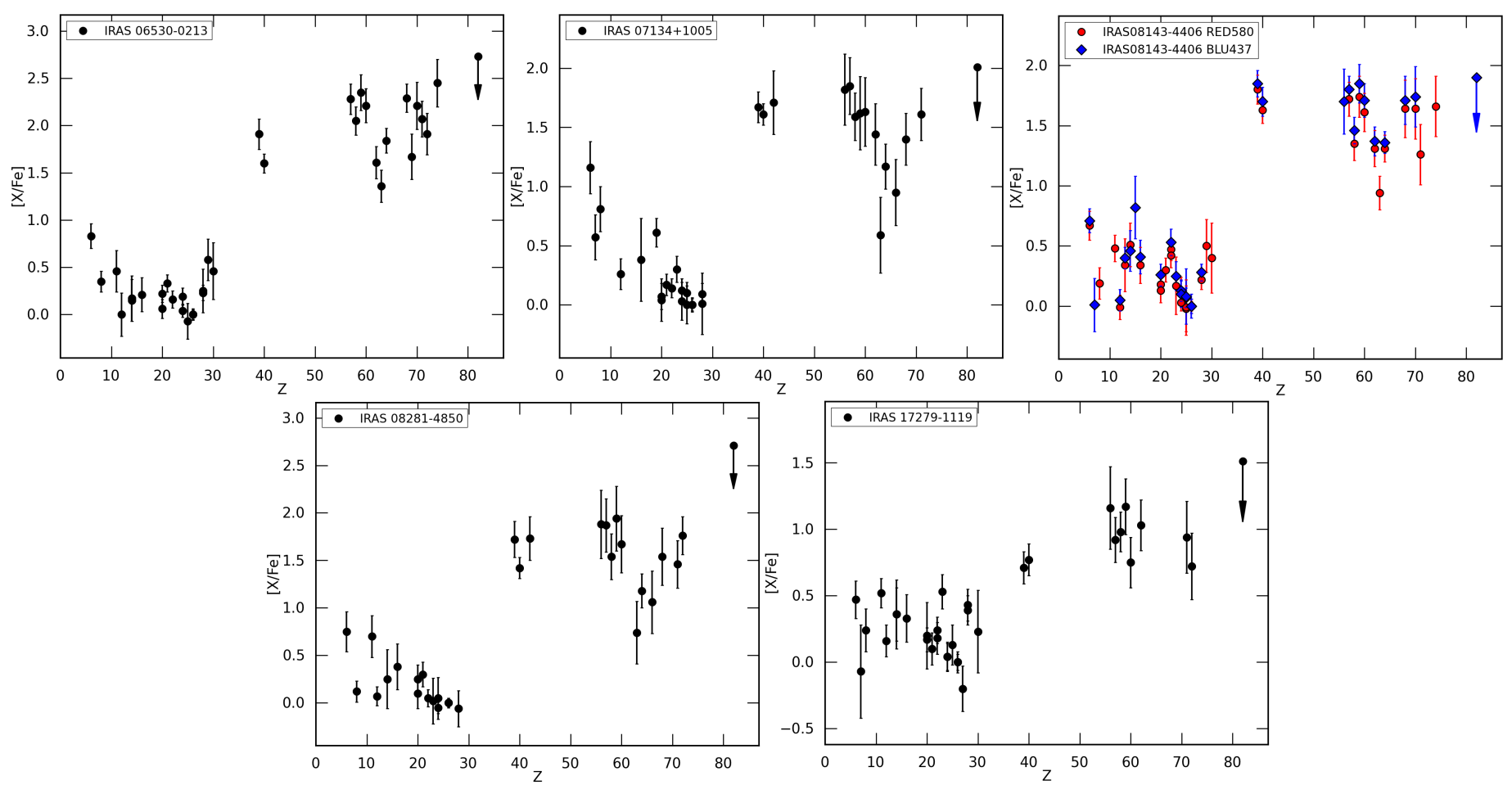

Fig. 9. Element-over-iron ratios for the programme stars with $7000 \mathrm{~K} \leqslant T_{\text {eff }}<8000 \mathrm{~K}$. The down arrow at $Z=82$ displays the adopted $\mathrm{Pb}$ abundance upper limits. For IRAS 08143-4406, the abundance results of both observational settings are shown.

Figure 13 shows the $\mathrm{Pb}$ abundance determination for IRAS 13245-5036 with the Pb II line at 5608.853 $\AA$. We do not detect any $\mathrm{Pb}$ II line feature for this star, and therefore we adopt an upper limit of the $\mathrm{Pb}$ abundance, which creates a synthetic $\mathrm{Pb}$ II line that lies slightly below the observed spectrum. The $\mathrm{Pb}$ I line at $4057.807 \AA$ would need a significantly higher $\mathrm{Pb}$ abundance than the adopted upper limit based on the
$\mathrm{Pb}$ II line. Therefore, we only consider the upper limit based on the $\mathrm{Pb}$ II line.

The determinations of the upper limits on the $\mathrm{Pb}$ abundances of all other programme stars are presented and discussed in Appendix $\mathrm{A}$. We do not detect strong $\mathrm{Pb}$ lines for any of the $14 s$-process enriched post-AGB stars. For the remaining part of the paper, we therefore refer to the $\mathrm{Pb}$ upper limit abundances as 
K. De Smedt et al.: Pb in Galactic s-process enriched post-AGB stars
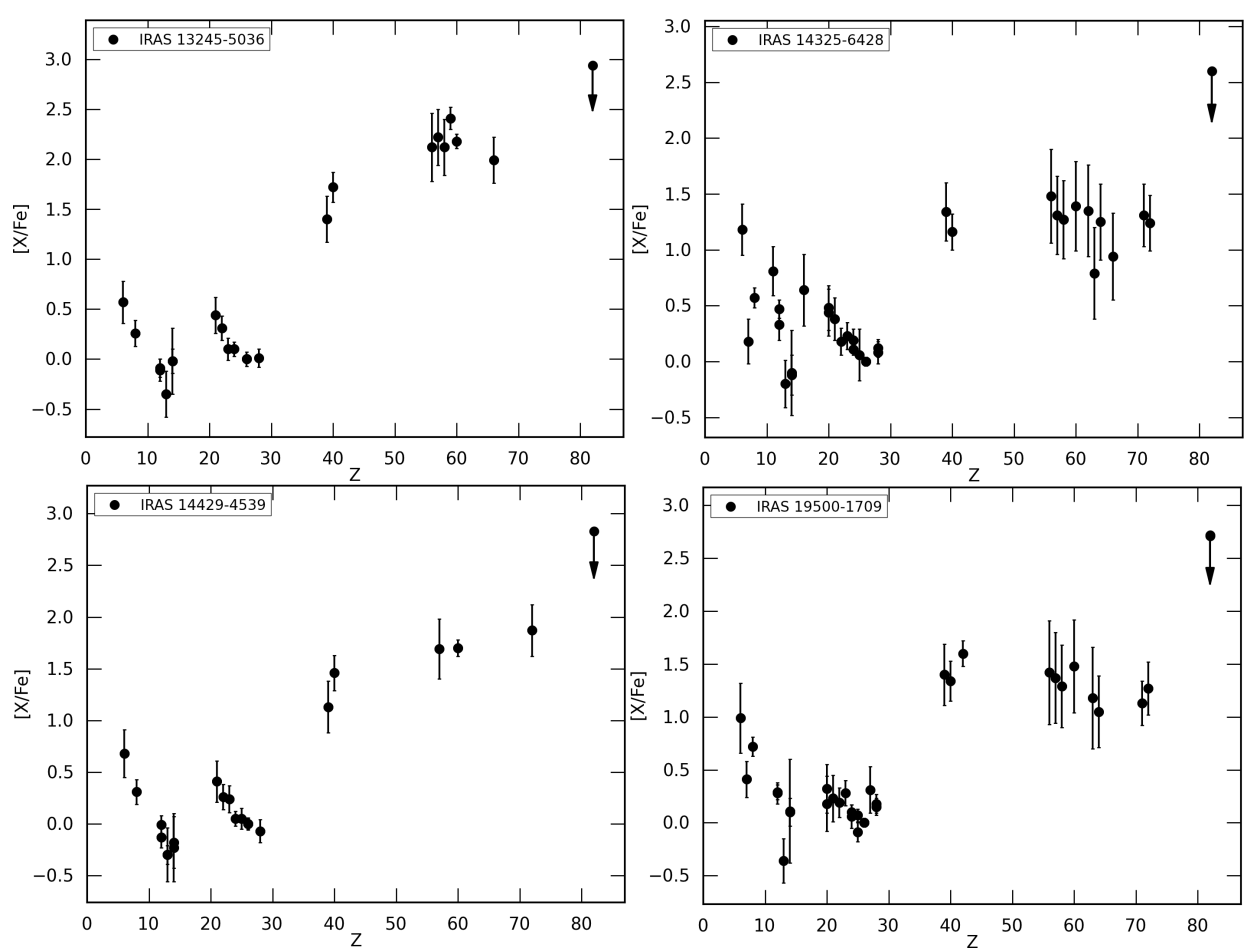

Fig. 10. Element-over-iron ratios for the programme stars with $T_{\mathrm{eff}} \geqslant 8000 \mathrm{~K}$. The down arrow at $Z=82$ displays the adopted $\mathrm{Pb}$ abundance upper limits.

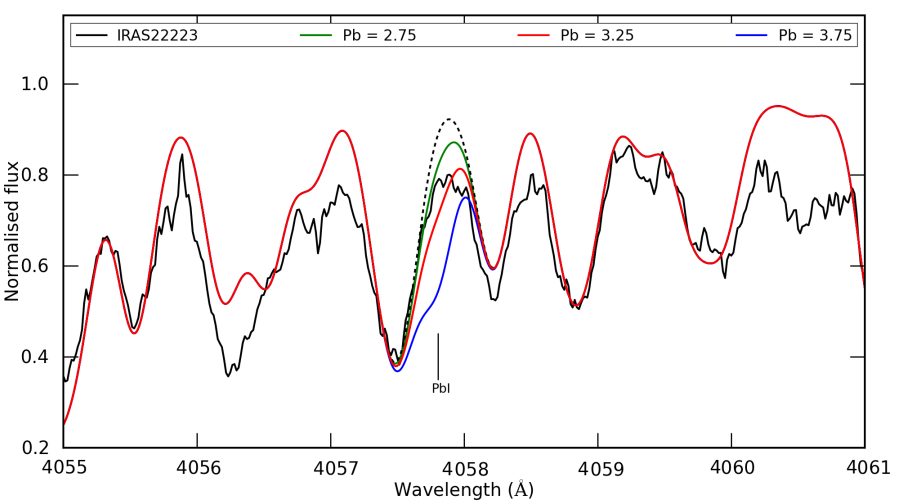

Fig. 11. Spectrum synthesis of the $\mathrm{Pb} \mathrm{I}$ line at $4057.807 \AA$ for IRAS $22223+4327$. The black spectrum is the observed HERMES spectrum, the coloured spectra represent synthetic spectra with different $\mathrm{Pb}$ abundances. The red line represents the adopted $\mathrm{Pb}$ abundance upper limit; the green and blue lines represent the adopted abundance upper limit -0.5 dex and +0.5 dex, respectively. The dashed black line shows the synthetic spectrum if no $\mathrm{Pb}$ is present. For more information, see text.

$\mathrm{Pb}_{\text {up }}$. In Table 7, we list the individual $\mathrm{Pb}$ upper limit abundance results for the $\mathrm{Pb} \mathrm{I}$ and $\mathrm{Pb}$ II line of the programme stars. The element-over-iron ratios corresponding to the adopted $\mathrm{Pb}$ abundance are shown in Figs. 8-10, and indicated with down arrows. The last six rows display the results of De Smedt et al. (2014, 2015) for six carbon and $s$-process enhanced post-AGB stars in the Magellanic Clouds and are used in the analysis of the neutron irradiation in Sect. 6.

\section{Discussion}

The lack of accurate distance determinations for the 14 programme stars hinders accurate luminosity determinations, which
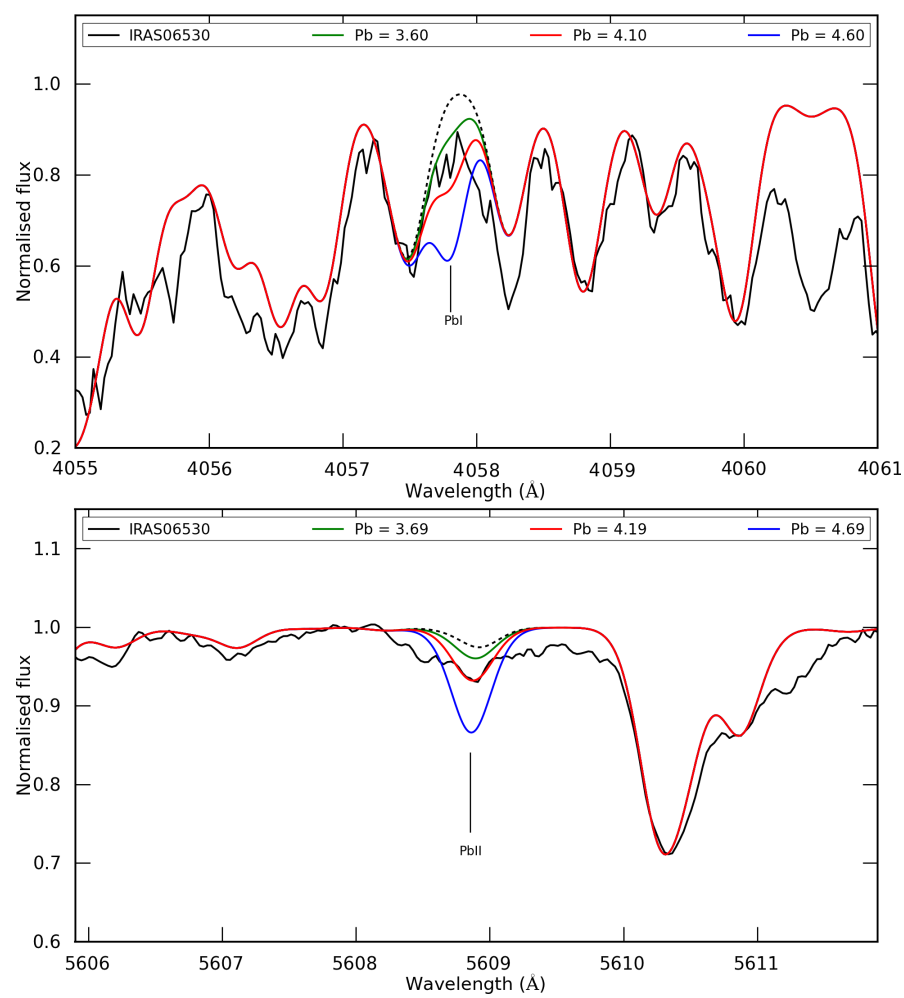

Fig. 12. Spectrum synthesis of the $\mathrm{Pb}$ I line at $4057.807 \AA$ (upper panel) and of the $\mathrm{Pb}$ II line at $5608.853 \AA$ (lower panel) for the UVES spectra of IRAS 06530-0213. Lines and symbols are similar to Fig. 11.

prevents us from estimating the core masses and from constraining the initial masses. Therefore, the comparison of our abundance results with tailored, state-of-the-art theoretical AGB evolution and nucleosynthetic models is difficult. Given the subsolar metallities we expect that the majority of the 14 sample stars will 
Table 7. $\mathrm{Pb}$ abundance upper limits $\mathrm{Pb}$ up of the programme stars, together with the $\mathrm{Pb}$ indices, $\left[\mathrm{Pb}_{\text {up }} / \mathrm{hs}\right]$ and $\left[\mathrm{Pb}_{\text {up }} / \mathrm{ls}\right]$, and the effective temperature.

\begin{tabular}{l|c|cc|cc|c|c|c}
\hline \hline Object & {$[\mathrm{Fe} / \mathrm{H}]$} & $\log \epsilon\left(\mathrm{PbI}_{\text {up }}\right)$ & {$\left[\mathrm{PbI}_{\text {up }} / \mathrm{Fe}\right]$} & $\log \epsilon\left(\mathrm{PbII}_{\text {up }}\right)$ & {$\left[\mathrm{PbII}_{\text {up }} / \mathrm{Fe}\right]$} & {$[\mathrm{Pb}$ up $/ \mathrm{hs}]$} & {$[\mathrm{Pb}$ up $/ \mathrm{ls}]$} & $T_{\text {eff }}(\mathrm{K})$ \\
\hline IRAS 05113+1347 & -0.49 & $<2.65$ & $<1.44$ & & & $<-0.21$ & $<0.11$ & 5500 \\
IRAS 05341+0852 & -0.54 & $<3.15$ & $<2.10$ & & & $<-0.14$ & $<0.23$ & 6750 \\
IRAS 06530-0213 & -0.32 & $<4.10$ & $<2.73$ & $<4.19$ & $<2.76$ & $<0.69$ & $<0.98$ & 7375 \\
IRAS 07134+1005 & -0.91 & $<2.79$ & $<2.01$ & & & $<0.38$ & $<0.37$ & 7250 \\
IRAS 07430+1115 & -0.31 & $<2.50$ & $<1.07$ & & & $<-0.48$ & $<-0.23$ & 6000 \\
IRAS 08143-4406 & -0.43 & $<3.20$ & $<1.90$ & & & $<0.32$ & $<0.13$ & 7000 \\
IRAS 08281-4850 & -0.26 & $<4.20$ & $<2.74$ & $<4.20$ & $<2.71$ & $<1.13$ & $<1.14$ & 7875 \\
IRAS 13245-5036 & -0.26 & & & $<4.40$ & $<2.94$ & $<0.91$ & $<1.38$ & 9500 \\
IRAS 14325-6428 & -0.56 & & & $<3.79$ & $<2.60$ & $<1.27$ & $<1.35$ & 8000 \\
IRAS 14429-4539 & -0.64 & & & $<.83$ & $<1.36$ & $<1.54$ & 9375 \\
IRAS 17279-1119 & -0.64 & $<2.75$ & $<1.51$ & & & $<0.59$ & $<0.77$ & 7250 \\
IRAS 19500-1709 & -0.59 & $<3.89$ & $<2.72$ & & $<1.38$ & $<1.35$ & 8000 \\
IRAS 22223+4327 & -0.30 & $<3.25$ & $<1.82$ & & $<0.94$ & $<0.48$ & 6500 \\
IRAS 22272+5435 & -0.77 & $<2.70$ & $<1.72$ & & & $<-0.18$ & $<0.11$ & 5750 \\
\hline J004441.04-732136.4 & -1.34 & $<3.00$ & $<2.58$ & & $<0.00$ & $<0.52$ & 6250 \\
J050632.10-714229.8 & -1.22 & $<2.05$ & $<1.52$ & & & $<0.45$ & $<0.10$ & 6750 \\
J052043.86-692341.0 & -1.15 & $<2.00$ & $<1.40$ & & & $<-0.45$ & $<-0.27$ & 5750 \\
J053250.69-713925.8 & -1.22 & $<2.23$ & $<1.70$ & & & $<-0.24$ & $<0.19$ & 5500 \\
J051213.81-693537.1 & -0.56 & $<3.30$ & $<2.11$ & & & $<0.37$ & $<0.78$ & 5875 \\
J051848.86-700246.9 & -1.03 & $<2.62$ & $<1.93$ & & & $<.19$ & $<0.47$ & 6000 \\
\hline
\end{tabular}

Notes. We adopt the solar $\mathrm{Pb}$ abundance of $\log \epsilon(\mathrm{Pb})=1.75$ dex from Asplund et al. (2009). The abundances of $\mathrm{Pb}$ I and $\mathrm{Pb}$ II are determined via the $4057.807 \AA$ and $5608.853 \AA$ spectral lines, respectively. The last six rows indicate the $\mathrm{Pb}_{\text {up }}$ results of six post-AGB stars in the Magellanic Clouds from De Smedt et al. $(2014,2015) .{ }^{(a)}$ Determined via the Blue437 spectra of IRAS 08143-4406.

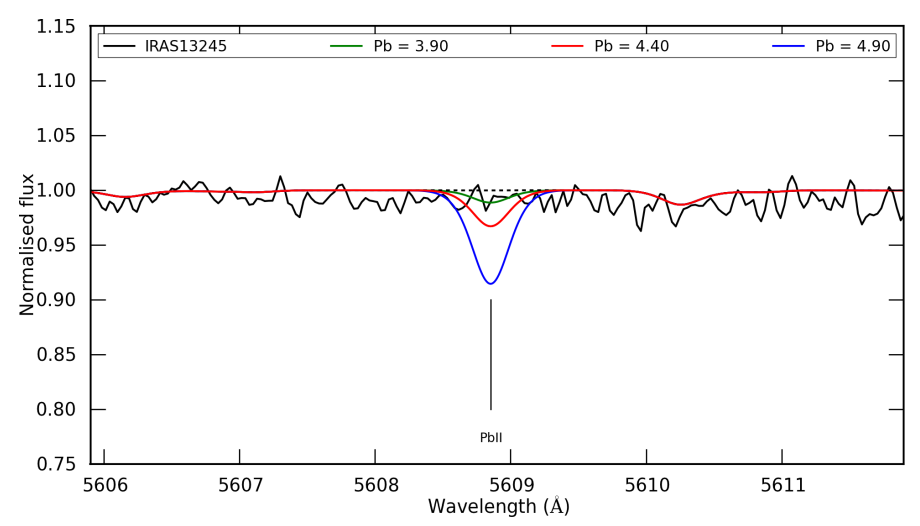

Fig. 13. Spectrum synthesis of the Pb II line at $5608.853 \AA$ for the UVES spectrum of IRAS 13245-5036. Lines and symbols are similar to Figs. 11 and 12.

have low initial masses $\left(M \leqslant 2.0 M_{\odot}\right)$. Future distance determinations will be essential to confirm this assumption.

\subsection{Abundance results versus general predictions}

In this section, we compare our derived $\mathrm{Pb}_{\text {up }}$ results with AGB model predictions obtained for a star of $2 M_{\odot}$. The $s$-process in present AGB models is almost insensitive to the initial stellar mass for these low-mass AGB stars.

Different observational indices are commonly used for representing $s$-process distributions and $s$-process overabundances, namely $[\mathrm{ls} / \mathrm{Fe}],[\mathrm{hs} / \mathrm{Fe}],[\mathrm{s} / \mathrm{Fe}]$, and $[\mathrm{hs} / \mathrm{ls}]$. The elements that are included for the calculation of these indices vary between authors. Here, we calculate the indices with the same elements as in De Smedt et al. (2015, and references therein). For the 1s index, we take the mean of the relative abundances of $\mathrm{Y}$ and $\mathrm{Zr}$. For hs, we compute the mean of the relative abundances of $\mathrm{La}, \mathrm{Ce}, \mathrm{Nd}$, and $\mathrm{Sm}$. The $[\mathrm{hs} / \mathrm{Fe}]$ and $[\mathrm{ls} / \mathrm{Fe}]$ of the programme stars are listed in Table 8 . The index $[\mathrm{s} / \mathrm{Fe}]$ is the mean of the relative abundances of the elements for the ls and hs indices and the index [hs/ls] is calculated according to $[\mathrm{hs} / \mathrm{Fe}]-[\mathrm{ls} / \mathrm{Fe}]$. The index [hs/ls] is an indicator of the neutron irradiation when the $s$-process takes place in radiative shells (Gallino et al. 1998; Busso et al. 2001). Larger neutron irradiation results in the creation of heavier $s$-process elements, hence, $[\mathrm{hs} / \mathrm{ls}]>0$. Indices $[\mathrm{Pb} / \mathrm{hs}]([\mathrm{Pb} / \mathrm{Fe}]-[\mathrm{hs} / \mathrm{Fe}])$ and $[\mathrm{Pb} / \mathrm{ls}]([\mathrm{Pb} / \mathrm{Fe}]-[\mathrm{ls} / \mathrm{Fe}])$ then represent the overabundance of $\mathrm{Pb}$ with respect to the other $s$-process overabundances. The third and second last column of Table 7 show the $\left[\mathrm{Pb}_{\text {up }} / \mathrm{hs}\right]$ and $\left[\mathrm{Pb}_{\text {up }} / \mathrm{ls}\right]$.

We were unable to determine the abundances of $\mathrm{Ce}$ for IRAS 14429-4539 and Sm in four hotter stars. To estimate the $\mathrm{Ce}$ and $\mathrm{Sm}$ abundances for these objects, we scale the abundances of $\mathrm{Ce}$ and $\mathrm{Sm}$ to the abundances of $\mathrm{La}$ and $\mathrm{Nd}$, which are the elements with an atomic mass closest to Ce and Sm. To scale the abundances, we make use of the AGB nucleosynthesis models from the online database FRUITY ${ }^{2}$ (Franec Repository of Upgraded Isotopic Tables and Yields; Cristallo et al. 2011), which covers a wide metallicity range, and we use the models with metallicities closest to the metallicity of the star. We use an initial mass of $1.5 M_{\odot}$ for the FRUITY models.

The $[\mathrm{s} / \mathrm{Fe}]$ results in Table 8 indeed confirm the strong $s$-process enhancements of the sample stars with $[\mathrm{s} / \mathrm{Fe}] \geqslant$ 1.0 except for IRAS $17279-1119$ with $[\mathrm{s} / \mathrm{Fe}]=0.86$ dex, which is mildly $s$-process enriched $(0.0<[\mathrm{s} / \mathrm{Fe}]<1.0 \mathrm{dex})$. IRAS $22223+4327$ and IRAS 08143-4406 have [hs/ls] $<0$. IRAS 07134+1005, IRAS 08281-4850, IRAS 14325-6428, and IRAS $19500-1709$ have [hs/ls] $\approx 0$, while the remaining eight stars, including the mildly enriched IRAS 17279-1119 have [hs/ls] $>0$.

The dominant neutron source in low-mass AGB stars is expected to be the ${ }^{13} \mathrm{C}(\alpha, n){ }^{16} \mathrm{O}$ reaction during the radiative interpulse phase, leading to [hs/ls] $>0$ at subsolar metallicities -1.2 dex $\lesssim[\mathrm{Fe} / \mathrm{H}] \lesssim-0.1$ dex (Goriely \& Mowlavi 2000). In

2 http://fruity.oa-teramo.inaf.it/ 
K. De Smedt et al.: Pb in Galactic s-process enriched post-AGB stars

Table 8. Overview of the $\mathrm{C} / \mathrm{O}$ ratio, the metallicity, and $s$-process indices for our sample of stars.

\begin{tabular}{|c|c|c|c|c|c|c|}
\hline Object & $\mathrm{C} / \mathrm{O}$ & {$[\mathrm{Fe} / \mathrm{H}]$} & {$[1 \mathrm{~s} / \mathrm{Fe}]$} & [hs/Fe] & {$[\mathrm{s} / \mathrm{Fe}]$} & [hs/ls] \\
\hline IRAS $05113+1347$ & $2.42 \pm 0.40$ & $-0.49 \pm 0.15$ & $1.33 \pm 0.13$ & $1.65 \pm 0.07$ & $1.54 \pm 0.07$ & $0.32 \pm 0.15$ \\
\hline IRAS $05341+0852$ & $06 \pm$ & $-0.54=$ & .08 & 2.24 & 2.12 & 0.10 \\
\hline IRAS 06530-0213 & $1.66 \pm 0.39$ & $-0.32 \pm 0.11$ & $1.75 \pm 0.09$ & $2.04 \pm 0.08$ & $1.94 \pm 0.06$ & 0.13 \\
\hline IRAS $07134+1005$ & $1.24 \pm 0.29$ & $-0.91 \pm 0.20$ & $1.64 \pm 0.13$ & $1.63 \pm 0.20$ & $1.63 \pm 0.14$ & $-0.01 \pm 0.24$ \\
\hline IRAS $07430+1115$ & $1.71 \pm 0.30$ & $-0.31 \pm 0.15$ & $1.30 \pm 0.14$ & $1.55 \pm 0.06$ & $1.47 \pm 0.06$ & $0.25 \pm 0.15$ \\
\hline IRAS 08143-4406 ${ }^{a}$ & $1.66 \pm 0.39$ & $-0.43 \pm 0.11$ & $1.77 \pm 0.08$ & $1.58 \pm 0.06$ & $1.65 \pm 0.05$ & $-0.19 \pm 0.11$ \\
\hline IRAS 08281-4850 & $2.34 \pm 0.42$ & $-0.26 \pm 0.11$ & $1.57 \pm 0.11$ & $1.58 \pm 0.12$ & $1.58 \pm 0.09$ & $0.01 \pm 0.17$ \\
\hline IRAS $13245-5036$ & $1.11 \pm 0.30$ & $-0.30 \pm 0.10$ & $1.56 \pm 0.14$ & $2.03 \pm 0.11$ & $1.88 \pm 0.09$ & $0.47 \pm 0.18$ \\
\hline IRAS $14325-6428$ & $2.27 \pm 0.40$ & $-0.56 \pm 0.10$ & $1.25 \pm 0.15$ & $1.33 \pm 0.19$ & $1.30 \pm 0.14$ & $0.08 \pm 0.24$ \\
\hline IRAS 14429-4539 & $1.29 \pm 0.26$ & $-0.18 \pm 0.11$ & $1.29 \pm 0.15$ & $1.47 \pm 0.10$ & $1.41 \pm 0.08$ & $0.18 \pm 0.08$ \\
\hline IRAS 17279 & $0.94 \pm 0.22$ & $-0.64 \pm 0.12$ & $0.74 \pm 0.08$ & $0.92 \pm 0.09$ & $0.86 \pm 0.07$ & 0.12 \\
\hline IRAS 19500-1709 & $1.02 \pm 0.17$ & $-0.59 \pm 0.10$ & $1.37 \pm 0.29$ & $1.34 \pm 0.30$ & $1.35 \pm 0.21$ & $-0.03 \pm 0.41$ \\
\hline IRAS $22223+4327$ & $1.04 \pm 0.22$ & $-0.30 \pm 0.11$ & $1.34 \pm 0.07$ & $0.88 \pm 0.07$ & $1.03 \pm 0.05$ & $-0.46 \pm 0.10$ \\
\hline IRAS $22272+5435$ & $1.46 \pm 0.26$ & $-0.77 \pm 0.12$ & $1.61 \pm 0.08$ & $1.90 \pm 0.07$ & $1.80 \pm 0.05$ & $0.28 \pm 0.11$ \\
\hline
\end{tabular}

Notes. ${ }^{(a)} \mathrm{C} / \mathrm{O}$ ratio is calculated from the results of the Red580 observations of IRAS 08143-4406; all other results are calculated from the results of the Blue437 observations.

intermediate-mass AGB stars $\left(M>\sim 4 M_{\odot}\right)$, the ${ }^{22} \mathrm{Ne}(\alpha, n)^{25} \mathrm{Mg}$ reaction within the convective thermal pulse may dominate the neutron production if the temperature at the base of the pulse reaches some $3.5 \times 10^{8} \mathrm{~K}$. In this case, the production of light $s$-process elements is favoured because of the convective nature of the region where the neutron irradiation takes place, and the resulting [hs/ls] index tends to be negative (e.g. Goriely \& Mowlavi 2000; Goriely \& Siess 2004; Karakas et al. 2012).

In Fig. 14, we compare the adopted $\mathrm{Pb}_{\text {up }}$ results with the $[\mathrm{Pb} / \mathrm{hs}]$ and $[\mathrm{Pb} / \mathrm{ls}]$ predictions of AGB evolution and nucleosynthesis models, as in De Smedt et al. (2015). The determined abundances are compared to the $2.0 M_{\odot}$ model predictions of the STAREVOL code (Goriely \& Siess 2004; Siess 2007, and references therein), the $2.0 M_{\odot}$ and $2.1 M_{\odot}$ Mount-Stromlo Evolutionary (MSE) predictions (Fishlock et al. 2014; Karakas 2010, and references therein), and the $2.0 M_{\odot}$ model predictions of FRUITY (Cristallo et al. 2011). The MSE, STAREVOL, and FRUITY predictions are rather insensitive to the initial stellar mass and show the global expected behaviour of $[\mathrm{Pb} / \mathrm{hs}]$ and $[\mathrm{Pb} / \mathrm{ls}]$ as a function of $[\mathrm{Fe} / \mathrm{H}]$. For the clarity of Fig. 14, we have omitted the $1.5 M_{\odot}$ FRUITY model data since the differences between the $1.5 M_{\odot}$ and $2.0 M_{\odot}$ models are small.

For higher metallicity stars with $[\mathrm{Fe} / \mathrm{H}]>-0.7$ dex, the model predictions are well within the observed $\mathrm{Pb}_{\text {up }}$ results, while for stars with $[\mathrm{Fe} / \mathrm{H}]<-0.7$ dex the discrepancy between observations and predictions become larger as the metallicity decreases. This trend is also confirmed by stars with well-constrained $\mathrm{Pb}_{\text {up }}$ results, such as IRAS $05113+1347$, IRAS 05341+0852, IRAS 06530-0213, and IRAS 22223+4327, covering an $[\mathrm{Fe} / \mathrm{H}]$ range from -0.8 dex to -0.3 dex. For IRAS $07430+1115$, the $\left[\mathrm{Pb}_{\text {up }} / \mathrm{hs}\right]$ result falls within the predicted limits.

In addition, it is not surprising to see that the $\mathrm{Pb}_{\text {up }}$ results of the hottest stars in the sample lie high above the model predictions in Fig. 14. As expected from Fig. 4, the very high $\mathrm{Pb}_{\text {up }}$ results for these stars are more related to temperature than to visible $\mathrm{Pb}$ line features. Therefore, we consider these $\mathrm{Pb}_{\text {up }}$ as poorly constrained. We conclude that stars with $T_{\text {eff }}>7500 \mathrm{~K}$ without significantly high $\mathrm{Pb}$ abundances are not useful for comparison with theoretical $\mathrm{Pb}$ abundance predictions. However, these hot stars remain useful for $\mathrm{C} / \mathrm{O}$ ratio and other $s$-element comparisons.

\subsection{Correlations}

Previous studies (e.g. Van Winckel 2003) have shown that there is no correlation between metallicity and neutron irradiation. According to the models, the [hs/ls] index is expected to increase with decreasing metallicity up to $[\mathrm{Fe} / \mathrm{H}] \sim-0.4$ dex after which it strongly decreases towards higher metallicities (e.g. Goriely \& Mowlavi 2000). With the addition of our new results, we confirm that this is not observed: in the lower panel of Fig. 15, we illustrate the absence of this expected correlation between metallicity and [hs/ls]. This implies that the total neutron irradiation is not (only) dependent on metallicity in our metallicity range.

In the upper panel of Fig. 15, we show the correlation between $[\mathrm{hs} / \mathrm{Fe}]$ and $[\mathrm{ls} / \mathrm{Fe}]$ for our sample stars, the Magellanic Cloud post-AGB stars in van Aarle et al. (2013), De Smedt et al. (2014, 2015), and for two additional Galactic $s$-process enriched post-AGB stars from Reyniers et al. (2004). It is clear that both $s$-process indices are correlated and a high $[1 \mathrm{~s} / \mathrm{Fe}]$ generally also implies a strong enhancement in [hs/Fe]. The spread is, however, large and this indicates that the different objects were subject to a variable neutron irradiation, irrespective of the dilution of the atmosphere.

In previous studies, Reyniers et al. (2004), van Aarle et al. (2013), and in De Smedt et al. (2015) identified a strong correlation between $[\mathrm{s} / \mathrm{Fe}]$ and the $[\mathrm{hs} / \mathrm{ls}]$ index in Galactic and Magellanic Cloud objects. Strongly enriched objects, for which there has been a low dilution of the enriched material with the envelope material, were also subject to strong neutron irradiation and, hence, showed a high [hs/ls]. Here, we add/update the results of our 14 programme stars to the previously used sample. The resulting $[\mathrm{s} / \mathrm{Fe}]$ and $[\mathrm{hs} / \mathrm{ls}]$ correlation is shown in the middle panel of Fig. 15 where two correlations are shown. The dashed line represents the correlation between the $[\mathrm{s} / \mathrm{Fe}]$ vs. [hs/ls] results for all stars in the plot; the full line represents the correlation without the inclusion of IRAS 172791119. We choose these two correlations as IRAS 17279-1119 has $[\mathrm{s} / \mathrm{Fe}]<1$ dex whereas the other stars have $[\mathrm{s} / \mathrm{Fe}] \geqslant 1$ dex. The correlation of the full and dashed lines in the middle panel of Fig. 15 are 0.75 and 0.65 , respectively. The second result shows that the inclusion of the mildly enhanced IRAS 172791119 moderately lowers the correlation for all objects. This suggests a different $s$-process history for IRAS 17279-1119 with respect to the other programme stars (see Sect. 7). 

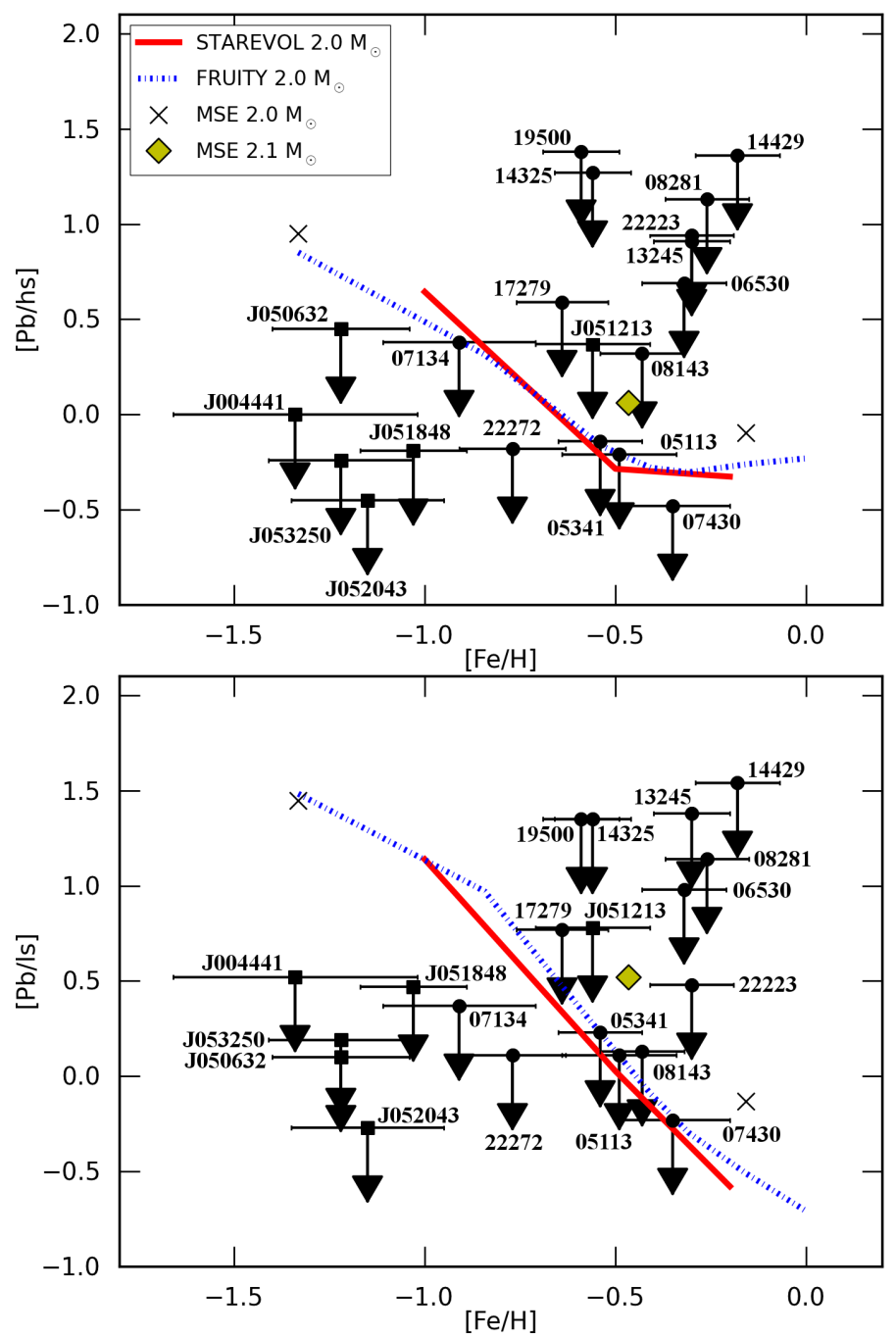

Fig. 14. The observed $\left[\mathrm{Pb}_{\mathrm{up}} / \mathrm{hs}\right]$ (upper panel) and the $\left[\mathrm{Pb}_{\mathrm{up}} / \mathrm{ls}\right]$ (lower panel) versus $[\mathrm{Fe} / \mathrm{H}]$ results of the Galactic sample stars of this study (black circles) and the Magellanic Cloud stars in De Smedt et al. (2014; 2015; black squares). The observed abundance upper limits are plotted together with the $[\mathrm{Pb} / \mathrm{hs}]$ and $[\mathrm{Pb} / \mathrm{ls}]$ predictions of the $2.0 M_{\odot}$ STAREVOL models (red full line), the 2.0 $M_{\odot}$ and $2.1 M_{\odot}$ Mount Stromlo models (MSE; black crosses and yellow diamond, respectively), and the $2.0 M_{\odot}$ FRUITY models (blue dot-dashed line). The black horizontal lines represent the $[\mathrm{Fe} / \mathrm{H}]$ uncertainty of the displayed stars. The position of each star from this study is indicated with the first numbers of its IRAS name; the first part of the 2MASS name is used for Magellanic Cloud stars from De Smedt et al. (2014, 2015).

\section{IRAS 17279-1119}

IRAS 17279-1119 has only a mild $s$-process enrichment (see lower right panel of Fig. 9) and is an outlier in the middle panel of Fig. 15, so in this section we focus specifically on this object.

Figure 16 shows the spectral energy distribution (SED) of IRAS 17279-1119. The photometric data for constructing the SED is retrieved from the following catalogues: the General Catalogue of Photometric Data (GCPD; Mermilliod et al. 1997), the All-sky compiled catalogue of 2.5 million stars (Kharchenko 2001), the 2MASS All-Sky Catalog of Point Sources (Cutri et al. 2003), the WISE All-Sky Data catalogue (Cutri et al. 2012), the AKARI/IRC mid-infrared all-sky survey (Ishihara et al. 2010), and the IRAS catalogue of Point Sources (Helou \& Walker 1988).
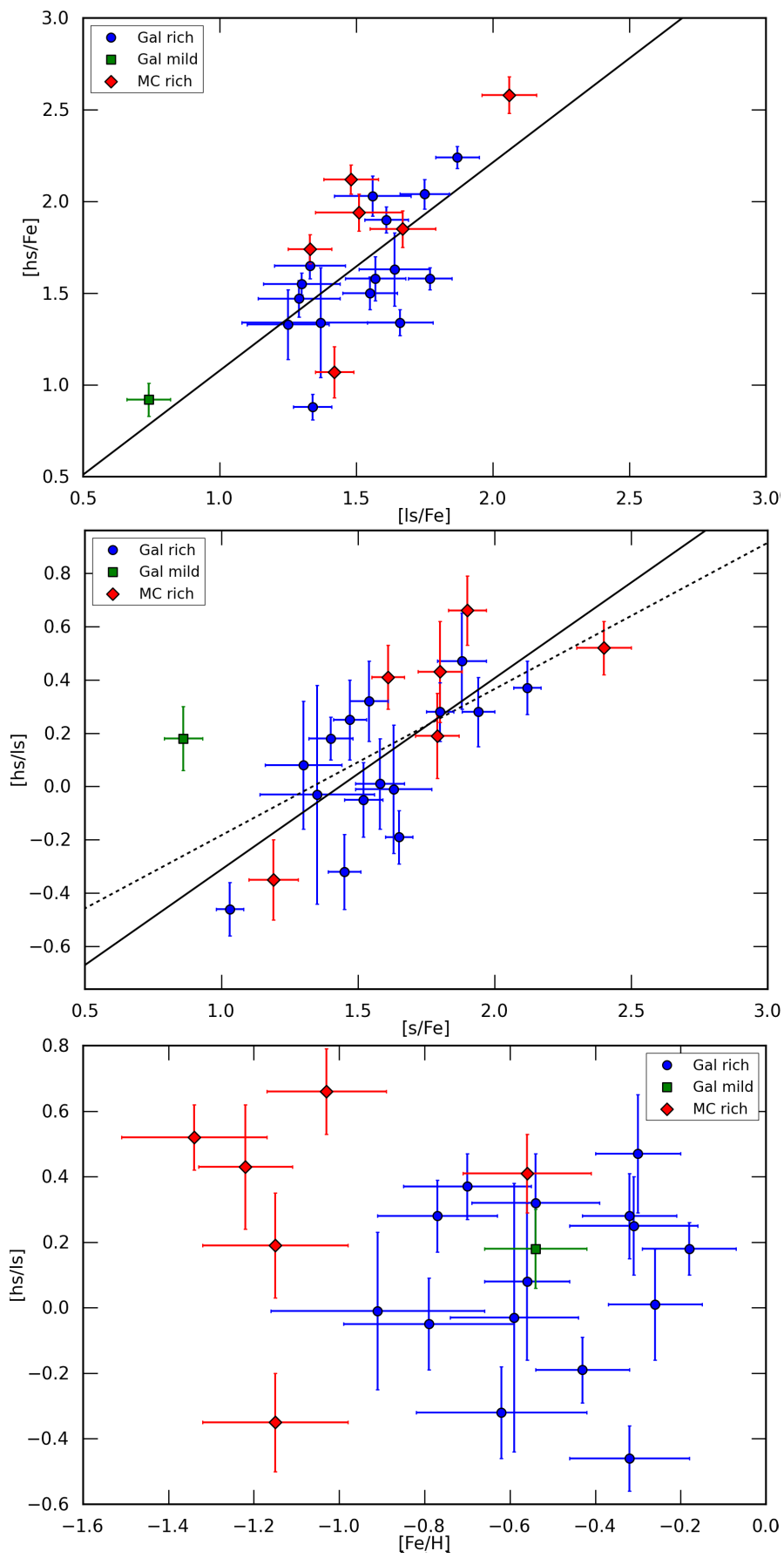

Fig. 15. Upper panel: correlation between the $s$-process indices $[\mathrm{hs} / \mathrm{Fe}]$ and $[1 \mathrm{~s} / \mathrm{Fe}]$ for our sample stars and Magellanic Cloud stars from De Smedt et al. $(2014,2015)$. Also included are the results of two Galactic s-process enriched stars from Reyniers et al. (2004): IRAS $04296+3429$ and IRAS $23304+6147$. Galactic objects are represented by blue circles (=strongly-enriched) and a green square (=mildly-enriched), Magellanic Cloud objects are represented by red diamonds. Middle panel: similar to upper panel but for the correlation between the total enrichment in $s$-process elements [s/Fe] and the [hs/ls] index. The dashed line shows the least-squares fit to all results, the full line shows the fit for all stars except for IRAS 17279-1119 (green square). Lower panel: similar to upper panel but for the correlation between the metallicity $[\mathrm{Fe} / \mathrm{H}]$ and the $[\mathrm{hs} / \mathrm{ls}]$ index. For more information, see text.

Figure 16 shows both the original raw photometric points (red diamonds) as well as the dereddened data (blue dots). We 


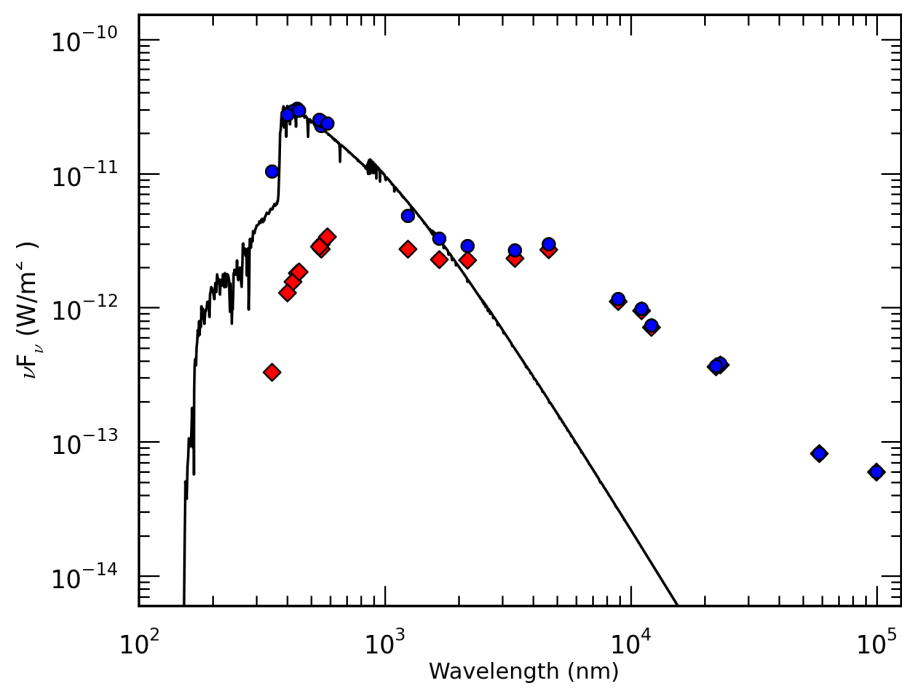

Fig. 16. Spectral energy distribution of IRAS 17279-1119. Red dots represent the red, original photometry, blue dots represent the dereddened photometry. The black line is the scaled Kurucz atmosphere model.

consider two possible reddening sources for Galactic objects. The first source is reddening by interstellar dust in the Galaxy towards IRAS 17279-1119. The second source is reddening by the circumstellar dust of IRAS 17279-1119 itself. We use the Galactic extinction curves of Cardelli et al. (1989) to determine the reddening for both sources. The total reddening is determined by applying a $\chi^{2}$ minimisation on the fit between the dereddened broadband fluxes and our preferred Kurucz model atmosphere, which we used for the abundance study of IRAS 17279-1119. The error on the total reddening $E(B-V)$ is determined by a Monte Carlo simulation of 100 arrays with a normal distribution of the original flux. We find a total reddening of $E(B-V)=0.76 \pm 0.02$ for IRAS 17279-1119.

The SED shown in Fig. 16 indicates an excess starting at 2 micron indicating the thermal emission of dust near the dustsublimation temperature and therefore close to the central star. It is now well established that this traces the presence of a stable compact circumbinary disc (e.g. De Ruyter et al. 2006; Deroo et al. 2007; Gielen et al. 2011; Hillen et al. 2013, 2015; Bujarrabal et al. 2015; Gezer et al. 2015). The specific characteristics of the SED are closely related to the binary nature of the central star (Van Winckel et al. 2009).

We obtained radial velocity monitoring data with the HERMES spectrograph (Raskin et al. 2011) mounted on the 1.2 meter Mercator telescope in the framework of our large programme on evolved binaries (Van Winckel et al. 2010; Gorlova et al. 2013). In total we obtained 85 radial velocity measurements starting on 19/06/2009 covering a total time span of 2211d. We also used the ASAS (Pojmanski \& Maciejewski 2004) photometry to study the photometric behaviour. The photometry consists of 788 data points covering a time span of 3156d. Many of the variables near the Population II Cepheid instability strip have complex light curves in which several modes are excited with periods close to each other so that beating effects are easily detectable (Kiss et al. 2006). IRAS 17279-1119 is no exception and periods of 90,87 , and 83 days are found in the photometry using a $\mathrm{S} / \mathrm{N}$ criterion of 4 in the Fourier transform. Unfortunately, our photometric series do not overlap in time with the radial velocity series.

We detect orbital motion (see Fig. 17) with an orbital period of exactly one year. The orbital solution is clear with a fractional

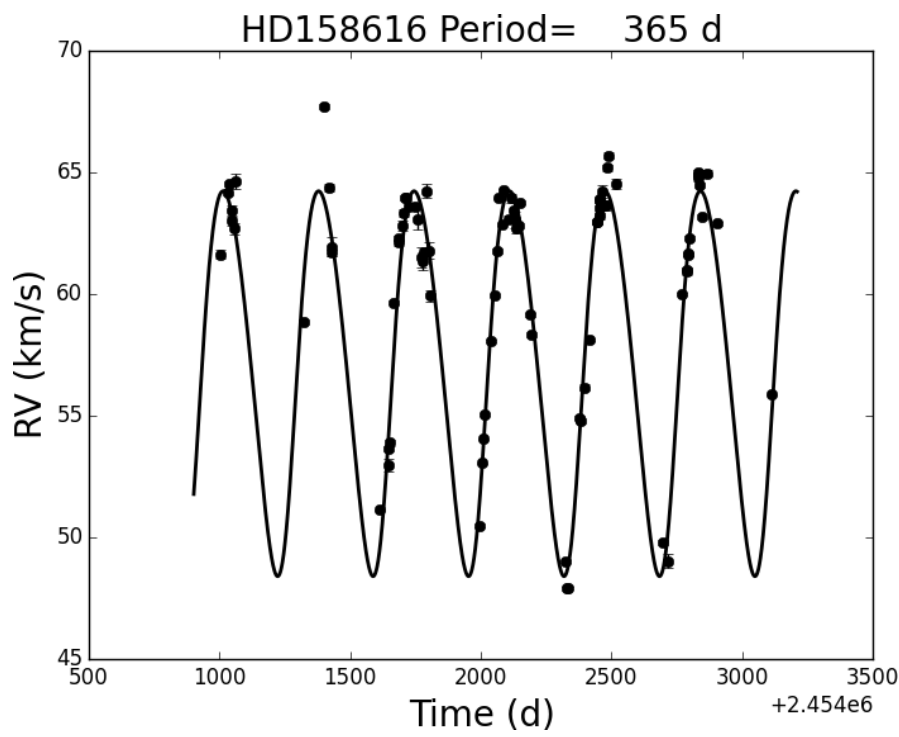

Fig. 17. Radial velocity orbit of IRAS 17279-1119. Black dots represent the observed radial velocities; the black curve is the sine-fit to the orbit. For more information, see text.

Table 9. Orbital elements of IRAS 17279-1119.

\begin{tabular}{lcc}
\hline \hline & & $\sigma$ \\
\hline Period $(\mathrm{d})$ & 365.0 & 1.0 \\
$e$ & 0.0 (forced) & \\
$K\left(\mathrm{~km} \mathrm{~s}^{-1}\right)$ & 7.92 & 0.44 \\
$\gamma\left(\mathrm{km} \mathrm{s}^{-1}\right)$ & 56.8 & 0.5 \\
$a \sin i(\mathrm{AU})$ & 0.27 & 0.02 \\
$f(M)\left(M_{\odot}\right)$ & 0.026 & 0.004 \\
$\mathrm{rms} \mathrm{residuals}\left(\mathrm{km} \mathrm{s}^{-1}\right)$ & 1.8 & \\
\hline
\end{tabular}

variance reduction of $91 \%$ in the fit. In Table 9 we list the orbital data. We cover in total close to six cycles, but as the period is close to one year, the phase coverage is not ideal. Nonetheless, the orbital detection is clear.

With an " $a \sin i$ " of only $0.27 \mathrm{AU}$, it is clear that the current orbit is too small to accommodate a TP-AGB star whose radius is expected to be 1-2 AU. The object must have experienced strong binary interaction when on the AGB. The mass function gives a constraint on the mass of the companion provided the mass of the primary and the inclination are known. As the primary is a post-AGB star, it is fair to assume the mass is a typically white dwarf mass of $0.6 M_{\odot}$. Assuming a most probable inclination of 60 degrees, the mass of the companion is only $0.3 M_{\odot}$. With an inclination of 30 degrees, this becomes $0.6 M_{\odot}$. As there are no symbiotic activities, we suggest that the companion is non-evolved.

To our knowledge, IRAS 17279-1119 is the first $s$-process rich post-AGB star discovered to be in a spectroscopic binary. If, during the binary interaction phase, there was mass transfer of enriched material to the secondary, the companion accreted $s$-process rich gas. This object seems therefore a precursor of extrinsically $s$-process enriched stars. These are binaries in which the observed $s$-process rich component was polluted by material expelled from their companion stars when they passed the AGB evolutionary phase and which are now white dwarfs (see Jorissen et al. 1998). IRAS 17279-1119 may very well be the first detected precursor of Ba dwarfs, given the low mass of the companion. 


\section{Conclusions}

In this paper, we presented a homogeneous abundance study of a sample of 14 Galactic $s$-process enriched post-AGB stars with the aim of determining $\mathrm{Pb}$ abundances. Our results confirm that two newly identified $21 \mu \mathrm{m}$ sources, IRAS $13245-5036$ and IRAS 14429-4539, are indeed carbon and $s$-process enriched and we confirm the post-AGB nature of both objects. Furthermore, we find a strong chemical diversity for the sample stars, ranging from only a mild $s$-process enrichment up to strong $s$-process enrichment.

None of the sample objects display clear distinctive $\mathrm{Pb}$ lines in their spectra and therefore we can only determine $\mathrm{Pb}$ abundance upper limits $\left(\mathrm{Pb}_{\mathrm{up}}\right)$. These $\mathrm{Pb}$ abundance upper limits are determined using synthetic spectra. A comparison with theoretical spectra reveals that some of the cooler stars in the sample display visible contributions of $\mathrm{Pb}$ in the unresolved blends. Because of the low $\mathrm{S} / \mathrm{N}$, it is impossible to determine the contribution and hence abundance accurately.

For the hotter stars with $T_{\text {eff }}>7500 \mathrm{~K}$, there are no visible $\mathrm{Pb}$ line contributions in the spectra. Therefore, these $\mathrm{Pb}_{\text {up }}$ results are poorly constrained, which makes hot post-AGB stars without drastically high $\mathrm{Pb}$ abundances not useful for the $\mathrm{Pb}$ comparison with theoretical AGB nucleosynthesis predictions.

A comparison of observed Galactic $\mathrm{Pb}_{\text {up }}$ abundances with theoretical $s$-process calculations of $\sim 2.0 M_{\odot}$ stellar models confirm the results of Magellanic Cloud stars from De Smedt et al. (2014, 2015). For metallicities $[\mathrm{Fe} / \mathrm{H}]>-0.7 \mathrm{dex}$, the predicted $\mathrm{Pb}$ abundances are well within the observed $\mathrm{Pb}_{\text {up }}$ results, while there exists an increasing discrepancy towards lower metallicities. Additional research is necessary to explain this trend.

We confirm that there is no correlation between neutron irradiation and metallicity in the metallicity range probed here. These results show that a wide spread in neutron irradiation is needed at all metallicities to explain these results. In comparative studies of extrinsically $s$-process enhanced objects, similar conclusions were obtained, even at lower metallicity (e.g. Bisterzo et al. 2012).

We confirm the correlation between $[\mathrm{hs} / \mathrm{ls}]$ and $[\mathrm{s} / \mathrm{Fe}]$ indicating the connection between strong neutron irradiation and overall photospheric enrichments. The mildly $s$-process enriched IRAS 17279-1119 does not follow this correlation because the orbital elements suggest that an evolutionary cut-off from the AGB was forced prematurely because of binary interaction: the current orbit is too small to accommodate an AGB star with a typical luminosity. In addition, IRAS 17279-1119 is to our knowledge the first confirmed $s$-process rich post-AGB star in a binary system.

In summary, we find an increasing discrepancy between observed and predicted $\mathrm{Pb}$ abundances towards lower metallicities. All objects with a metallicity $[\mathrm{Fe} / \mathrm{H}]<-0.7$ dex show $\mathrm{Pb}$ upper limits that are lower than the predicted $\mathrm{Pb}$ abundances. Contrary to the Magellanic Cloud samples, the distances to the Galactic sample stars are poorly known and hence it is difficult to constrain their current core masses and their initial masses. Distance estimates are hence necessary to connect the determined abundance results with tailored theoretical predictions in detail. The GAIA distance determinations will be crucial in this respect.

Acknowledgements. The authors thank S. Cristallo for the public availability of the FRUITY models. K.D.S., H.V.W., R.M., and D.K. acknlowledge support of the KU Leuven contract GOA/13/012. D.K. acknowledges support of the FWO grant G.OB86.13. S.G. and L.S. are FNRS research associates. A.I.K. was supported through an Australian Research Council Future Fellowship
(FT110100475). Based on observations obtained with the HERMES spectrograph, which is supported by the Research Foundation - Flanders (FWO), Belgium, the Research Council of KU Leuven, Belgium, the Fonds National de la Recherche Scientifique (F.R.S.-FNRS), Belgium, the Royal Observatory of Belgium, the Observatoire de Genève, Switzerland and the Thüringer Landessternwarte Tautenburg, Germany.

\section{References}

Abia, C., Domínguez, I., Gallino, R., et al. 2002, ApJ, 579, 817

Abia, C., de Laverny, P., \& Wahlin, R. 2008, A\&A, 481, 161

Aoki, W., Ryan, S. G., Norris, J. E., et al. 2001, ApJ, 561, 346

Asplund, M., Grevesse, N., Sauval, A. J., \& Scott, P. 2009, ARA\&A, 47, 481

Behara, N. T., Bonifacio, P., Ludwig, H.-G., et al. 2010, A\&A, 513, A72

Bisterzo, S., Gallino, R., Straniero, O., Cristallo, S., \& Käppeler, F. 2010, MNRAS, 404, 1529

Bisterzo, S., Gallino, R., Straniero, O., Cristallo, S., \& Käppeler, F. 2012, MNRAS, 422, 849

Bujarrabal, V., Castro-Carrizo, A., Alcolea, J., \& Van Winckel, H. 2015, A\&A, 575, L7

Busso, M., Gallino, R., Lambert, D. L., Travaglio, C., \& Smith, V. V. 2001, ApJ, 557,802

Cardelli, J. A., Clayton, G. C., \& Mathis, J. S. 1989, ApJ, 345, 245

Castelli, F., \& Kurucz, R. L. 2004, Proc. IAU Symp. 210, poster A20 [arXiv: astro-ph/0405087]

Cerrigone, L., Hora, J. L., Umana, G., et al. 2011, ApJ, 738, 121

Cristallo, S., Piersanti, L., Straniero, O., et al. 2011, ApJS, 197, 17

Cutri, R. M., Skrutskie, M. F., van Dyk, S., et al. 2003, VizieR Online Data Catalog: II/246

Cutri, R. M., Wright, E. L., Conrow, T., et al. 2012, Explanatory Supplement to the WISE All-Sky Data Release Products, Tech. Rep.

De Ruyter, S., Van Winckel, H., Maas, T., et al. 2006, A\&A, 448, 641

De Smedt, K., Van Winckel, H., Karakas, A. I., et al. 2012, A\&A, 541, A67

De Smedt, K., Van Winckel, H., Kamath, D., et al. 2014, A\&A, 563, L5

De Smedt, K., Van Winckel, H., Kamath, D., \& Wood, P. R. 2015, A\&A, 583, A56

Dekker, H., D’Odorico, S., Kaufer, A., Delabre, B., \& Kotzlowski, H. 2000, in SPIE Conf. Ser. 4008, eds. M. Iye \& A. F. Moorwood, 534

Deroo, P., Reyniers, M., Van Winckel, H., Goriely, S., \& Siess, L. 2005, A\&A, 438, 987

Deroo, P., Acke, B., Verhoelst, T., et al. 2007, A\&A, 474, L45

Fishlock, C. K., Karakas, A. I., Lugaro, M., \& Yong, D. 2014, ApJ, 797, 44

Gallino, R., Arlandini, C., Busso, M., et al. 1998, ApJ, 497, 388

Gezer, I., Van Winckel, H., Bozkurt, Z., et al. 2015, MNRAS, 453, 133

Gielen, C., Bouwman, J., van Winckel, H., et al. 2011, A\&A, 533, A99

Goriely, S., \& Mowlavi, N. 2000, A\&A, 362, 599

Goriely, S., \& Siess, L. 2004, A\&A, 421, L25

Gorlova, N., Van Winckel, H., Vos, J., et al. 2013, in EAS PS 64, eds. K. Pavlovski, A. Tkachenko, \& G. Torres, 163

Helou, G., \& Walker, D. W. 1988, Infrared astronomical satellite (IRAS) catalogs and atlases, The small scale structure catalog, vol. 7

Hillen, M., Verhoelst, T., Van Winckel, H., et al. 2013, A\&A, 559, A111

Hillen, M., Menu, J., de Vries, B. L., et al. 2015, in Why Galaxies Care about AGB Stars III: A Closer Look in Space and Time, eds. F. Kerschbaum, R. F. Wing, \& J. Hron, ASP Conf. Ser., 497, 175

Hrivnak, B. J., Volk, K., \& Kwok, S. 2009, ApJ, 694, 1147

Ishihara, D., Onaka, T., Kataza, H., et al. 2010, A\&A, 514, A1

Jorissen, A., Van Eck, S., Mayor, M., \& Udry, S. 1998, A\&A, 332, 877

Kamath, D., Wood, P. R., \& Van Winckel, H. 2014, MNRAS, 439, 2211

Kamath, D., Wood, P. R., \& Van Winckel, H. 2015, MNRAS, 454, 1468

Karakas, A. I. 2010, MNRAS, 403, 1413

Karakas, A. I., \& Lattanzio, J. C. 2014, PASA, 31, 30

Karakas, A. I., García-Hernández, D. A., \& Lugaro, M. 2012, ApJ, 751, 8

Kharchenko, N. V. 2001, Kinematika i Fizika Nebesnykh Tel, 17, 409

Kiselman, D. 2002, Highlights of Astronomy, 12, 429

Kiss, L. L., Szabó, G. M., \& Bedding, T. R. 2006, MNRAS, 372, 1721

Kobayashi, C., Karakas, A. I., \& Umeda, H. 2011, MNRAS, 414, 3231

Kupka, F., Piskunov, N., Ryabchikova, T. A., Stempels, H. C., \& Weiss, W. W. 1999, A\&AS, 138, 119

Kwok, S., Volk, K. M., \& Hrivnak, B. J. 1989, ApJ, 345, L51

Lugaro, M., Karakas, A. I., Stancliffe, R. J., \& Rijs, C. 2012, ApJ, 747, 2

Lugaro, M., Campbell, S. W., Van Winckel, H., et al. 2015, A\&A, 583, A77

Lyubimkov, L. S., Lambert, D. L., Korotin, S. A., et al. 2011, MNRAS, 410, 1774

Mermilliod, J.-C., Mermilliod, M., \& Hauck, B. 1997, A\&AS, 124, 349

Mowlavi, N., Jorissen, A., \& Arnould, M. 1998, A\&A, 334, 153

Neyskens, P., van Eck, S., Jorissen, A., et al. 2015, Nature, 517, 174 
K. De Smedt et al.: Pb in Galactic s-process enriched post-AGB stars

Pojmanski, G., \& Maciejewski, G. 2004, Acta Astron., 54, 153

Rao, S. S., Giridhar, S., \& Lambert, D. L. 2012, MNRAS, 419, 1254

Raskin, G., van Winckel, H., Hensberge, H., et al. 2011, A\&A, 526, A69

Reddy, B. E., Bakker, E. J., \& Hrivnak, B. J. 1999, ApJ, 524, 831

Reddy, B. E., Lambert, D. L., Gonzalez, G., \& Yong, D. 2002, ApJ, 564, 482

Reyniers, M. 2002, Ph.D. Thesis, Belgium

Reyniers, M., Van Winckel, H., Gallino, R., \& Straniero, O. 2004, A\&A, 417, 269

Reyniers, M., Abia, C., Van Winckel, H., et al. 2007a, A\&A, 461, 641

Reyniers, M., van de Steene, G. C., van Hoof, P. A. M., \& van Winckel, H. 2007b, A\&A, 471, 247

Romano, D., Karakas, A. I., Tosi, M., \& Matteucci, F. 2010, A\&A, 522, A32

Siess, L. 2007, A\&A, 476, 893
Sneden, C. A. 1973, Ph.D. Thesis, The University of Texas at Austin Straniero, O., Gallino, R., Busso, M., et al. 1995, ApJ, 440, L85

van Aarle, E., van Winckel, H., Lloyd Evans, T., et al. 2011, A\&A, 530, A90

van Aarle, E., Van Winckel, H., De Smedt, K., Kamath, D., \& Wood, P. R. 2013 , A\&A, 554, A106

Van Eck, S., Goriely, S., Jorissen, A., \& Plez, B. 2001, Nature, 412, 793

Van Eck, S., Goriely, S., Jorissen, A., \& Plez, B. 2003, A\&A, 404, 291

Van Winckel, H. 2003, ARA\&A, 41, 391

Van Winckel, H., \& Reyniers, M. 2000, A\&A, 354, 135

Van Winckel, H., Lloyd Evans, T., Briquet, M., et al. 2009, A\&A, 505, 1221

Van Winckel, H., Jorissen, A., Gorlova, N., et al. 2010, MemSAI, 81, 1022 


\section{Appendix $\mathrm{A}: \mathrm{Pb}$ abundances of individual stars}

In this Appendix, we give an overview of the $\mathrm{Pb}$ abundance upper limit determinations for the different programme stars together with a brief discussion of the $\mathrm{Pb}_{\text {up }}$ results.

\section{A.1. IRAS $05113+1347$}

The $\mathrm{Pb}$ abundance upper limit determination of IRAS $05113+1347$ is presented in Fig. A.1. The determination of the continuum is difficult as the region is dominated by large spectral blends. We use the spectral lines at 4057.5 and $4058.2 \AA$ to optimally estimate the position of the continuum. Thereafter, we adopted a $\mathrm{Pb}$ abundance upper limit, which fully incorporates the line feature at $4057.807 \AA$.

The adopted $\mathrm{Pb}_{\text {up }}$ of IRAS $05113+1347$ results in a $\left[\mathrm{Pb}_{\text {up }} / \mathrm{Fe}\right]$ which is about 0.2 dex lower than the value for [hs/Fe] (left upper of panel Fig. 8) and similar to the element-over-iron ratios of the elements beyond the Ba-peak.

\section{A.2. IRAS $05341+0852$}

For the strongly $s$-process enriched IRAS $05341+0852$, we estimate the position of the continuum by finding a compromise between the spectral blends at 4057.5 and $4058.2 \AA$ in Fig. A.2. We adopt a $\mathrm{Pb}$ abundance upper limit, which fully includes the small visible line feature.

Although it is one of the most $s$-process enriched Galactic post-AGB stars studied to date, IRAS $05341+0852$ displays a $\left[\mathrm{Pb}_{\text {up }} / \mathrm{Fe}\right]$ that is about 0.15 dex lower than [hs/Fe] (middle upper panel of Fig. 8). Like in IRAS $05113+1347$, the $\left[\mathrm{Pb}_{\text {up }} / \mathrm{Fe}\right]$ seems similar to the element-over-iron ratios of the elements beyond the Ba-peak.

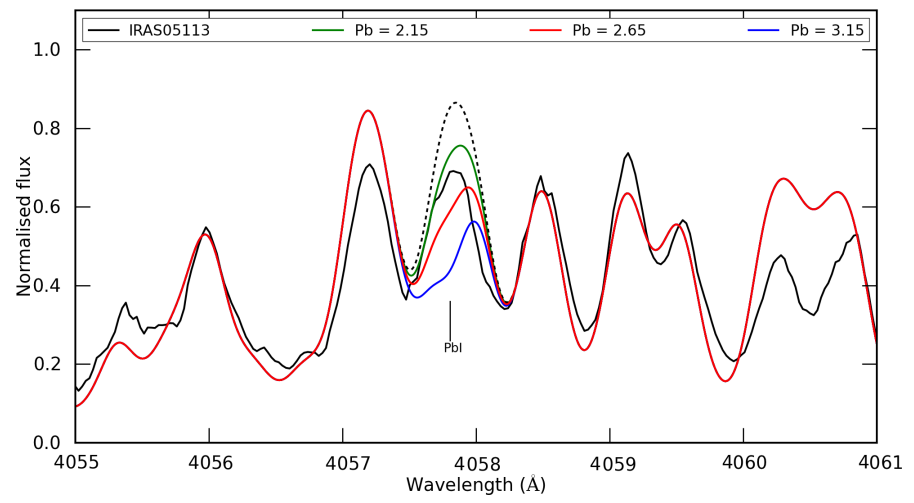

Fig. A.1. Spectrum synthesis of the $\mathrm{Pb}$ I line at $4057.807 \AA$ for IRAS $05113+1347$. The black spectrum is the observed UVES spectrum; the coloured spectra represent synthetic spectra with different $\mathrm{Pb}$ abundances. The red line represents the adopted $\mathrm{Pb}$ abundance upper limit; the green and blue lines represent the adopted abundance upper limit -0.5 dex and +0.5 dex, respectively. The dashed black line shows the synthetic spectrum if no $\mathrm{Pb}$ is present.

\section{A.3. IRAS 06530-0213}

The $\mathrm{Pb}$ abundance determination of IRAS 06530-0213 is described in detail in Sect. 5. The spectral synthesis of the $\mathrm{Pb}$ regions are depicted in Fig. 12. We use the $\mathrm{Pb}_{\text {up }}$ result of the $\mathrm{Pb}$ I line for this strongly $s$-process enriched post-AGB star. The

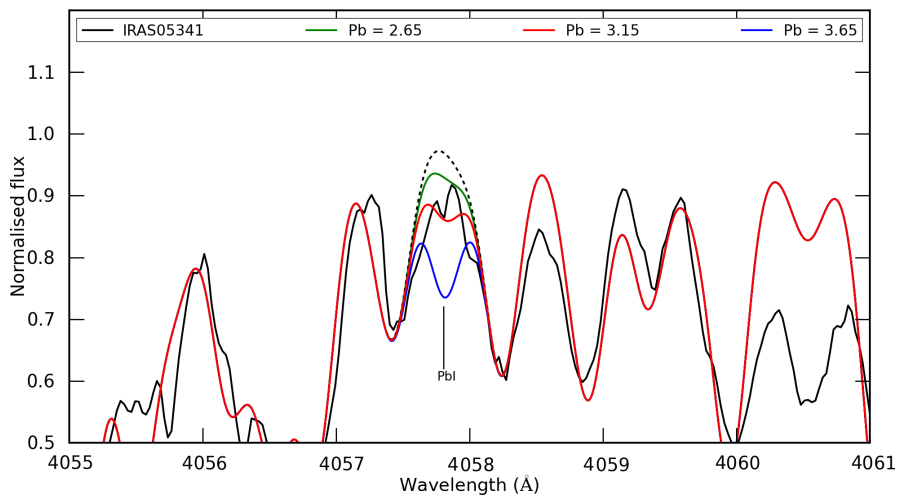

Fig. A.2. Spectrum synthesis of the Pb I line at $4057.807 \AA$ for the UVES spectrum of IRAS $05341+0852$. Lines and symbols are similar to Fig. A.1.

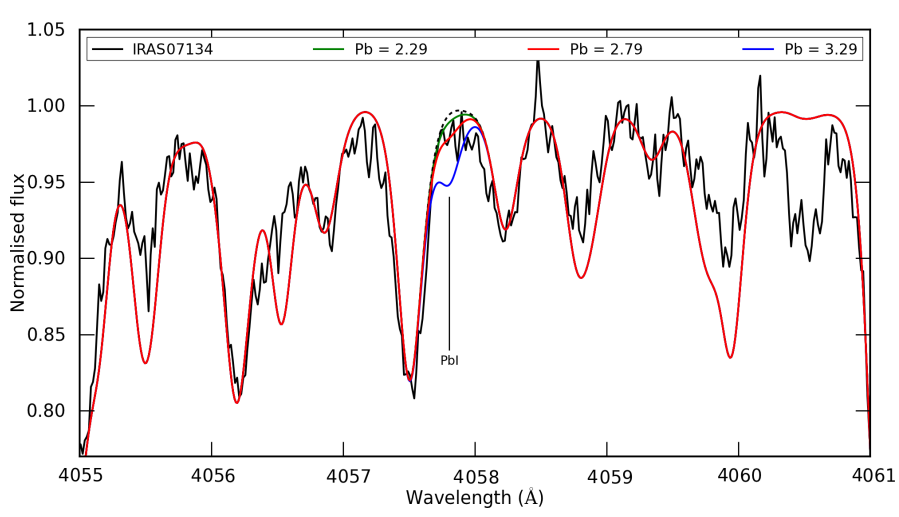

Fig. A.3. Spectrum synthesis of the Pb I line at $4057.807 \AA$ for the HERMES spectrum of IRAS $07143+1005$. Lines and symbols are similar to Fig. A.1.

resulting $\left[\mathrm{Pb}_{\mathrm{up}} / \mathrm{Fe}\right]$ is about 0.7 dex higher than $[\mathrm{hs} / \mathrm{Fe}]$ (left upper panel of Fig. 9) and the element-over-iron ratios of the elements beyond the Ba-peak.

\section{A.4. IRAS $07143+1005$}

We estimate the position of the continuum of IRAS $07143+1005$ using the spectral blends at 4056.2, 4056.9 and $4057.5 \AA$ at the blue side of the $\mathrm{Pb}$ I line, and the blends at 4058.2 and $4059.3 \AA$ at the red side of the $\mathrm{Pb}$ I line in Fig. A.3. The spectral region of the $\mathrm{Pb}$ I line is dominated by noise and we cannot detect any visible contribution from the $\mathrm{Pb}$ line. We therefore adopt a $\mathrm{Pb}$ abundance upper limit, which includes the noise at $4057.807 \AA$.

The adopted $\left[\mathrm{Pb}_{\mathrm{up}} / \mathrm{Fe}\right]$ is about 0.4 dex higher than $[\mathrm{hs} / \mathrm{Fe}]$ and $[1 \mathrm{~s} / \mathrm{Fe}]$, which are both similar in enrichment (middle upper panel of Fig. 9).

\section{A.5. IRAS $07430+1115$}

In Fig. A.4, we use the spectral blends at 4057.5 and $4058.8 \AA$ to estimate the position of the continuum. Although not clearly visible, the synthetic spectrum without the $\mathrm{Pb}$ I line suggests a possible small contribution of $\mathrm{Pb}$ at $4057.807 \AA$. Therefore, we adopt a $\mathrm{Pb}$ abundance upper limit that incorporates the peak of the $\mathrm{Pb}$ I line feature.

The adopted $\mathrm{Pb}_{\text {up }}$ of IRAS $07430+1115$ gives a $\left[\mathrm{Pb}_{\text {up }} / \mathrm{Fe}\right]$, which is significantly lower than $[\mathrm{hs} / \mathrm{Fe}]$ with a difference of about 0.5 dex (right upper panel of Fig. 8). 


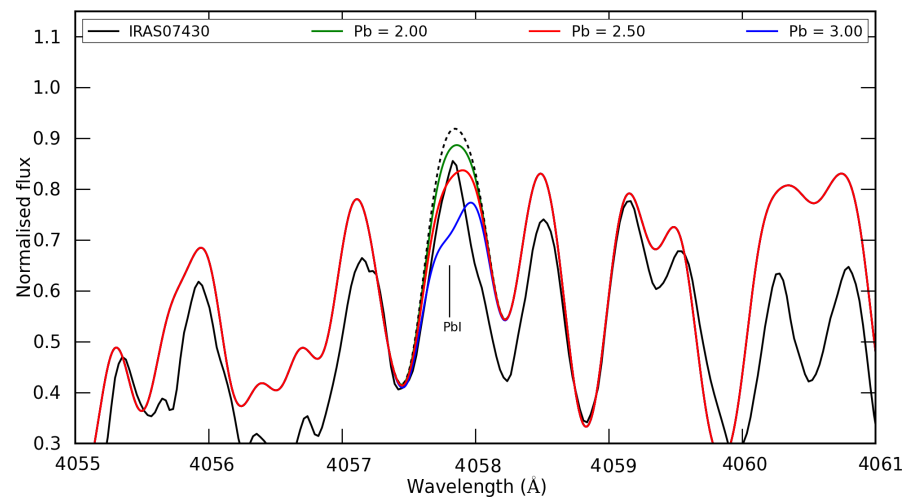

Fig. A.4. Spectrum synthesis of the $\mathrm{Pb}$ I line at $4057.807 \AA$ for the UVES spectrum of IRAS $07430+1115$. Lines and symbols are similar to Fig. A.1.

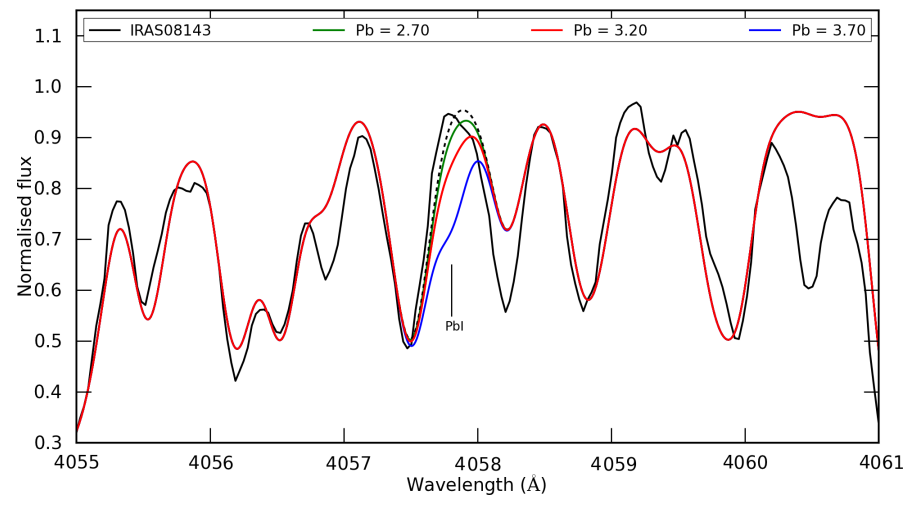

Fig. A.5. Spectrum synthesis of the $\mathrm{Pb}$ I line at $4057.807 \AA$ for the BLUE437 UVES spectrum of IRAS 08143-4406. Lines and symbols are similar to Fig. A.1.

\section{A.6. IRAS 08143-4406}

The position of the continuum for IRAS 08143-4406 is estimated using the spectral blends at 4057.5 and $4058.8 \AA$ in Fig. A.5. The spectrum of IRAS 08143-4406 does not show visible contribution of $\mathrm{Pb}$ at $4057.807 \AA$, and we see that with the estimated continuum position, the spectral feature at the blue side of the the $\mathrm{Pb}$ I line is not even contained within the boundaries of the synthetic spectrum without $\mathrm{Pb}$ I line, which could possibly be attributed to noise. As a result of these uncertainties, we adopt $\mathrm{a} \mathrm{Pb}$ abundance upper limit that fully incorporates the spectral line in the spectral region of the $\mathrm{Pb} \mathrm{I}$ line.

We find that $\left[\mathrm{Pb}_{\mathrm{up}} / \mathrm{Fe}\right]$ is about 0.1 higher than $[\mathrm{ls} / \mathrm{Fe}]$ and 0.3 dex higher than $[\mathrm{hs} / \mathrm{Fe}]$, respectively (right upper panel of Fig. 9).

\section{A.7. IRAS 08281-4850}

The spectra of IRAS08281-4850 reveal possible $\mathrm{Pb}$ line features in both panels of Fig. A.6, but we assume these features are more likely due to noise than a real abundance of $\mathrm{Pb}$. Nevertheless, we adopt a similar $\mathrm{Pb}$ abundance upper limit for both $\mathrm{Pb}$ lines that fully incorporates the visible spectral features. For the $\mathrm{Pb} \mathrm{I}$ line, we use the spectral blends at 4056.2, 4057.5, and $4058.8 \AA$ to estimate the position of the continuum. For the $\mathrm{Pb}$ II line, we see unidentified spectral lines at 5606.7 and $5607 \AA$ and also at both sides of the $\mathrm{Pb}$ II line. These spectral lines are not included in the linelists and we can only use the continuum in the region
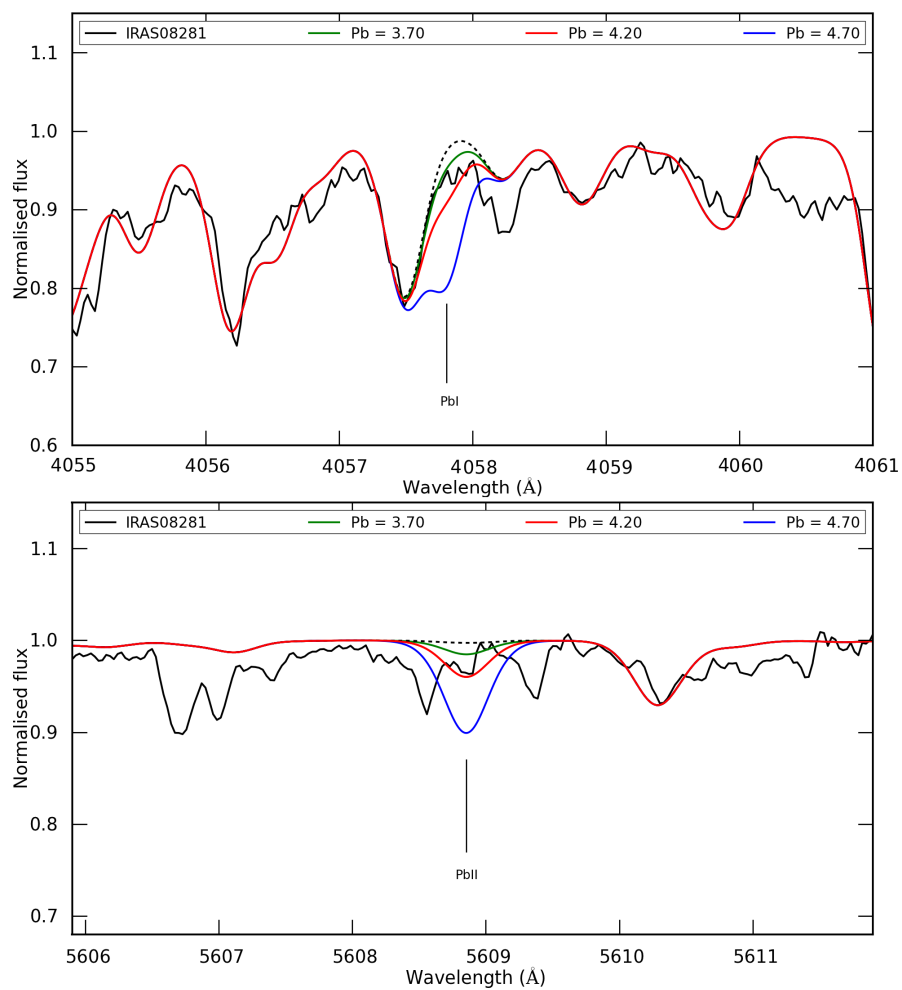

Fig. A.6. Spectrum synthesis of the $\mathrm{Pb}$ I line at $4057.807 \AA$ (upper panel) and the $\mathrm{Pb}$ II line at $5608.853 \AA$ (lower panel) for the UVES spectra of IRAS 08281-4850. Lines and symbols are similar to Fig. A.1.

5606-5606.5 $\AA$ and 5611.5-5612 $\AA$, in combination with the spectral line at $5610.2 \AA$, to estimate the continuum position.

We find a similar $\mathrm{Pb}_{\text {up }}$ for both the $\mathrm{Pb}$ I and $\mathrm{Pb}$ II lines. This results in a $\left[\mathrm{Pb}_{\mathrm{up}} / \mathrm{Fe}\right]$ that is about 1.2 dex higher than the [hs/Fe] and $[1 \mathrm{~s} / \mathrm{Fe}]$ (left lower panel of Fig. 9).

\section{A.8. IRAS $13245-5036$}

The $\mathrm{Pb}$ abundance determination of IRAS 13245-5036 is described in detail in Sect. 5. The spectral synthesis of the Pb II region is depicted in Fig. 13. For this hottest star in the sample, we only use the $\mathrm{Pb}$ II line to determine $\mathrm{Pb}_{\text {up }}$. The adopted $\left[\mathrm{Pb}_{\text {up }} / \mathrm{Fe}\right]$ is about 0.9 dex higher than $[\mathrm{hs} / \mathrm{Fe}$ ] (left upper panel of Fig. 10).

\section{A.9. IRAS $14325-6428$}

The $\mathrm{Pb}$ II line spectral region of IRAS $14325-6428$ is dominated by noise and does not show a visible $\mathrm{Pb}$ line contribution in Fig. A.7. We estimate the position of the continuum by fitting to the average continuum in the shown spectral region. Around $5610.2 \AA$, a small line appears in the synthetic spectra that is not present in the observed spectra, so we do not use this line for the continuum estimate. We then adopt a $\mathrm{Pb}$ abundance upper limit, which incorporates the continuum at $5608.853 \AA$.

The adopted $\left[\mathrm{Pb}_{\text {up }} / \mathrm{Fe}\right]$ is only determined via the $\mathrm{Pb}$ II line, and is about 1.3 dex higher than [hs/Fe] (right upper panel of Fig. 10). 


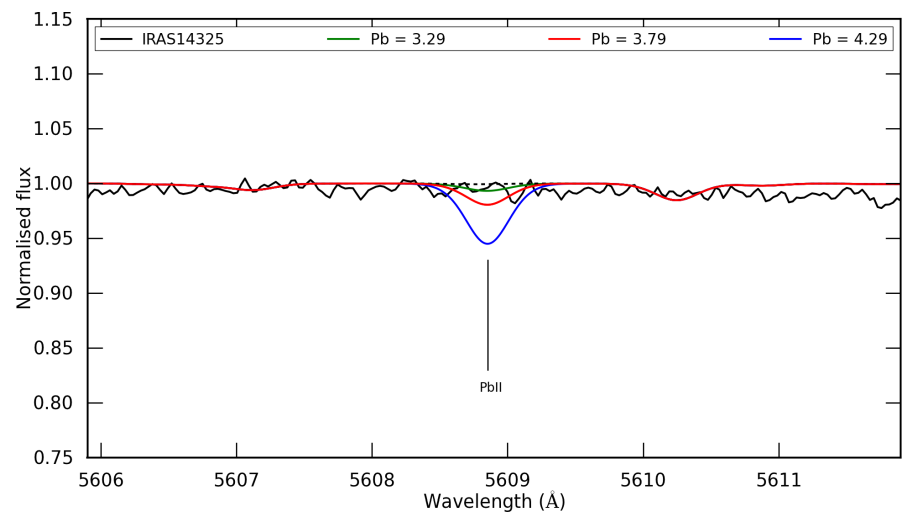

Fig. A.7. Spectrum synthesis of the Pb II line at $5608.853 \AA$ for the UVES spectrum of IRAS 14325-6428. Lines and symbols are similar to Fig. A.1.

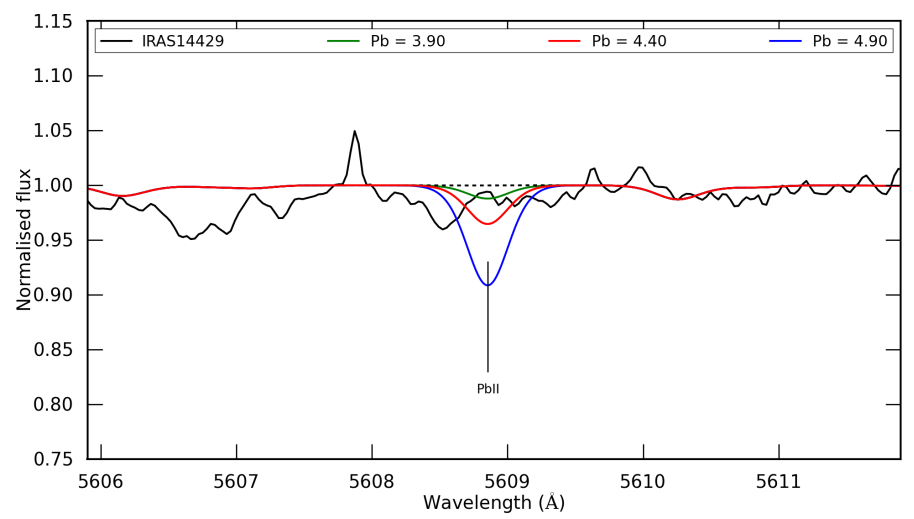

Fig. A.8. Spectrum synthesis of the Pb II line at $5608.853 \AA$ for the UVES spectrum of IRAS 14429-4539. Lines and symbols are similar to Fig. A.1.

\section{A.10. IRAS 14429-4539}

Figure A.8 shows that the spectral region of the $\mathrm{Pb}$ II at $5608.853 \AA$ of IRAS $14429-4539$ is totally dominated by noise and that there is no visible contribution of the $\mathrm{Pb}$ line. There is a visible line in the spectrum at $5606.6 \AA$, but it is not clear whether this feature is real or due to noise. For this reason, it is not included in the linelists. We therefore estimate the position of the continuum by fitting the average continuum in the shown spectral region. We then adopt a $\mathrm{Pb}$ abundance upper limit, which incorporates the estimated continuum at $5608.853 \AA$.

For the second hottest star in the sample, we only use the $\mathrm{Pb}$ II line for the $\mathrm{Pb}_{\text {up }}$ determination. $\left[\mathrm{Pb}_{\mathrm{up}} / \mathrm{Fe}\right]$ exceeds $[\mathrm{hs} / \mathrm{Fe}]$ by almost 1.4 dex (left lower panel of Fig. 10).

\section{A.11. IRAS 17279-1119}

The mildly IRAS $17279-1119$ does not show any visible $\mathrm{Pb}$ line features in Fig. A.9. The local continuum is estimated using the spectral blends at 4056.2, 4057.5 and 4058.2 $\AA$. We adopt a $\mathrm{Pb}$ abundance upper limit, which fully incorporates the spectral region at $4057.807 \AA$.

The $\left[\mathrm{Pb}_{\mathrm{up}} / \mathrm{Fe}\right]$ for the mildly $s$-process enriched post-AGB star IRAS 17279-1119 is 0.6 dex higher than [hs/Fe] (right lower panel of Fig. 9).

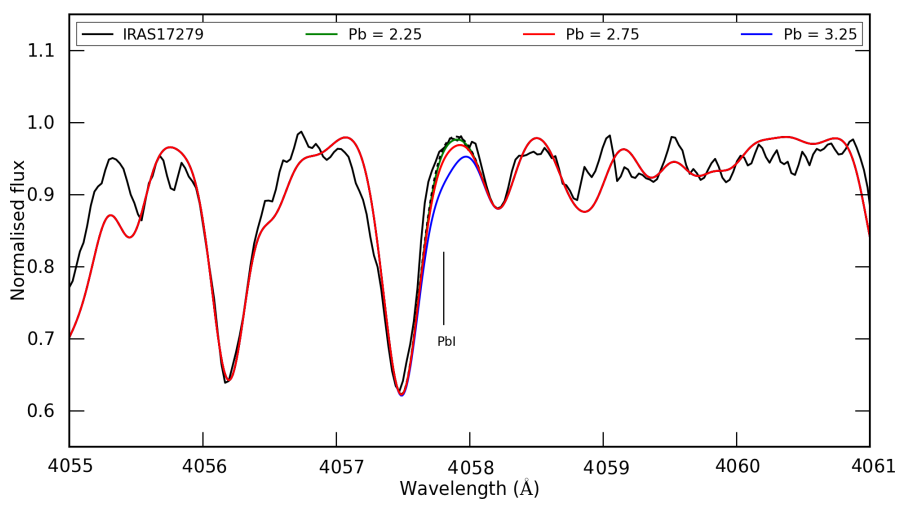

Fig. A.9. Spectrum synthesis of the $\mathrm{Pb}$ I line at $4057.807 \AA$ for the UVES spectrum of IRAS 17279-1119. Lines and symbols are similar to Fig. A.1.

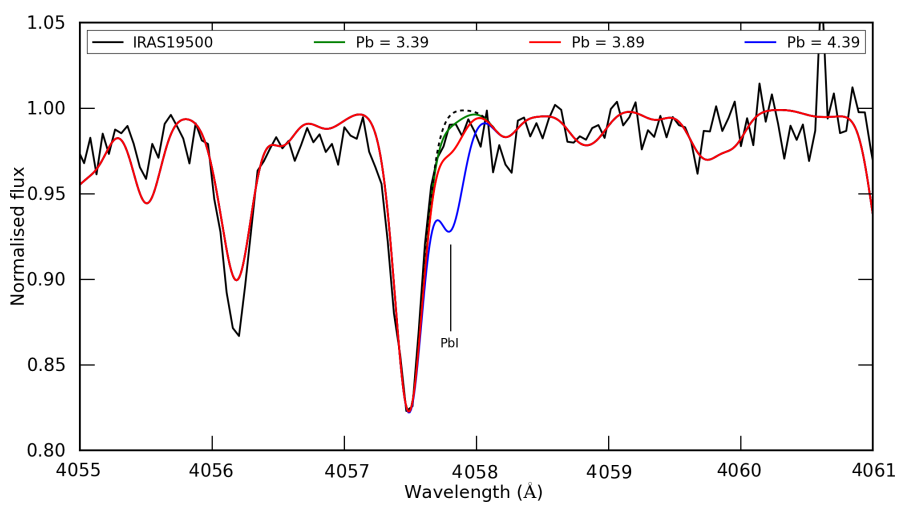

Fig. A.10. Spectrum synthesis of the Pb I line at $4057.807 \AA$ for the HERMES spectrum of IRAS 19500-1709. Lines and symbols are similar to Fig. A.1.

\section{A.12. IRAS 19500-1709}

For the atmospheric parameter set of IRAS 19500-1709 to appear, the $\mathrm{Pb}$ I line at $4057.807 \AA$ requires a lower $\mathrm{Pb}$ abundance than the Pb II line at $5608.853 \AA$. Therefore, we only use the $\mathrm{Pb}$ I line for our analysis. Figure A.10 shows that the spectral region of the $\mathrm{Pb}$ I line has a poor $\mathrm{S} / \mathrm{N}$. We use the spectral blend at $4057.5 \AA$ to estimate the position of the continuum and then adopt a $\mathrm{Pb}$ abundance upper limit, which fully incorporates the noise at $4057.807 \AA$.

For IRAS $19500-1709,[\mathrm{hs} / \mathrm{ls}] \approx 0$ and the derived $\left[\mathrm{Pb}_{\text {up }} / \mathrm{Fe}\right]$ is about 1.35 dex higher than $[\mathrm{ls} / \mathrm{Fe}]$ and [hs/Fe] (right lower panel of Fig. 10).

\section{A.13. IRAS $22223+4327$}

The $\mathrm{Pb}$ abundance determination of IRAS $22223+4327$ is described in detail in Sect. 5. The spectral synthesis of the $\mathrm{Pb}$ I region is depicted in Fig. 11. For IRAS 22223+4327, the adopted $\mathrm{Pb}_{\text {up }}$ results in a $\left[\mathrm{Pb}_{\text {up }} / \mathrm{Fe}\right]$, which lies about 1.0 dex above [hs/Fe] (left lower panel of Fig. 8). For IRAS 22223+4327, the ls elements have higher enrichments than the hs elements and our estimate for $\left[\mathrm{Pb}_{\mathrm{up}} / \mathrm{Fe}\right]$ lies about 0.5 dex above $[\mathrm{ls} / \mathrm{Fe}]$.

\section{A.14. IRAS $22272+5435$}

The spectral region of the $\mathrm{Pb}$ I line for IRAS $22272+5435$ is dominated by strong blends in combination with low $\mathrm{S} / \mathrm{N}$ in 


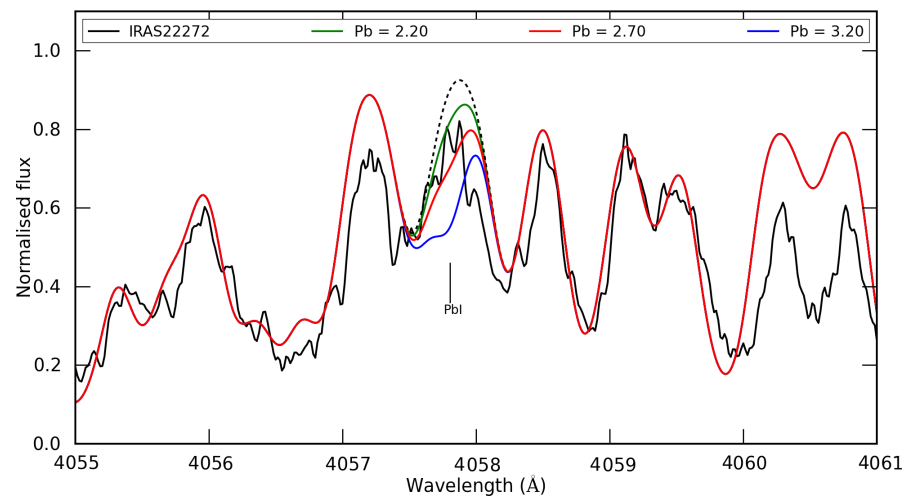

Fig. A.11. Spectrum synthesis of the $\mathrm{Pb} \mathrm{I}$ line at $4057.807 \AA$ for the HERMES spectrum of IRAS $22272+5435$. Lines and symbols are similar to Fig. A.1.

Fig. A.11. We use the spectral blends at 4057.5 and $4058.8 \AA$ to estimate the position of the continuum. We then adopt a Pb abundance upper limit that fully includes the visible line feature at $4057.807 \AA$.

The adopted $\left[\mathrm{Pb}_{\mathrm{up}} / \mathrm{Fe}\right]$ of IRAS $22272+5435$ lies between the results of $[\mathrm{hs} / \mathrm{Fe}]$ and [ls/Fe] (right lower panel of Fig. 8). The element-over-iron ratios of the elements beyond the Ba-peak are also slightly higher than $\left[\mathrm{Pb}_{\mathrm{up}} / \mathrm{Fe}\right]$.

\section{Appendix B: Comparison to previous studies}

In this Appendix, we present the comparisons of our derived abundances with those of previous studies. In this paper we rely on high-quality data and perform strictly homogeneous abundance analyses based on the same methods and atomic data. The comparison with the literature is overall satisfactory but some noticeable differences occur, especially when the original data were of inferior quality to our UVES and Hermes data.

Objects IRAS $05113+1347$ and IRAS $22272+5435$ have been previously studied by Reddy et al. (2002); the comparisons between the abundance results are presented in Figs. B.1 and B.2. We find similar sets of atmospheric parameters although our derived metallicity for IRAS $05113+1347$ is approximately 0.2 dex higher. We find a higher C/O ratio for IRAS $05113+1347$ but lower abundances for $\mathrm{Y}$ and the heavy s-elements. In partic-

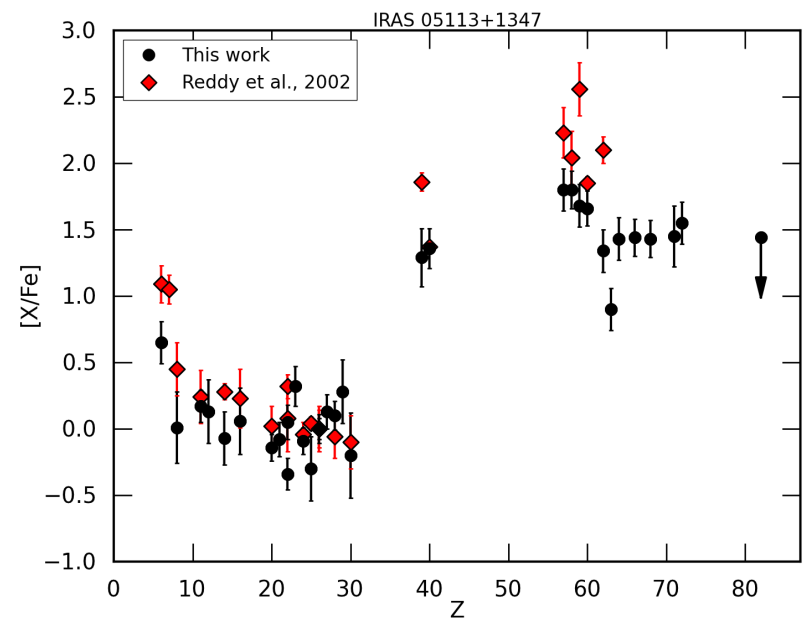

Fig. B.1. Comparison between our derived abundances and the results of Reddy et al. (2002) for IRAS $05113+1347$.

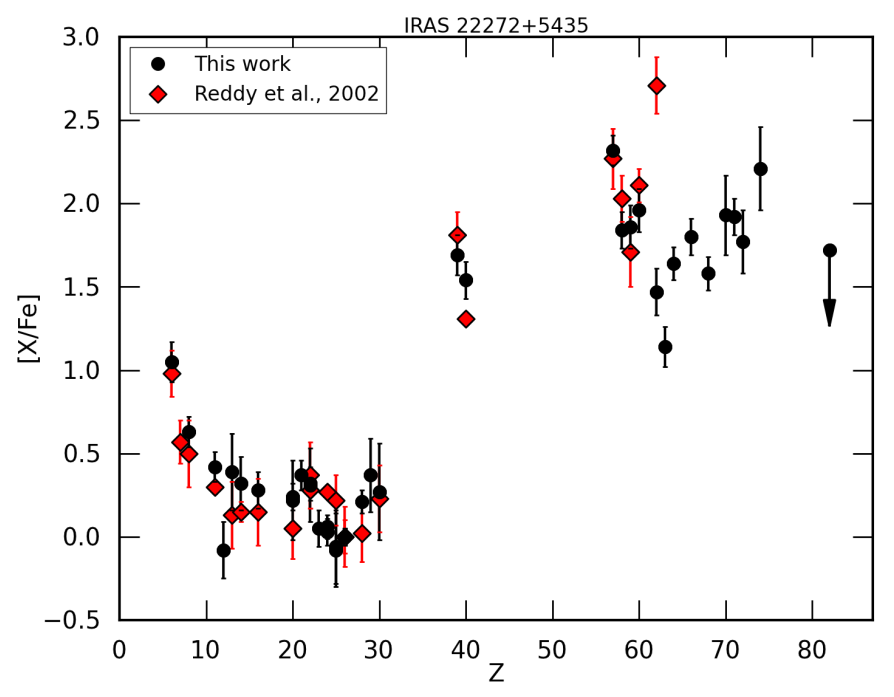

Fig. B.2. Comparison between our derived abundances and the results of Reddy et al. (2002) for IRAS $22272+5435$.

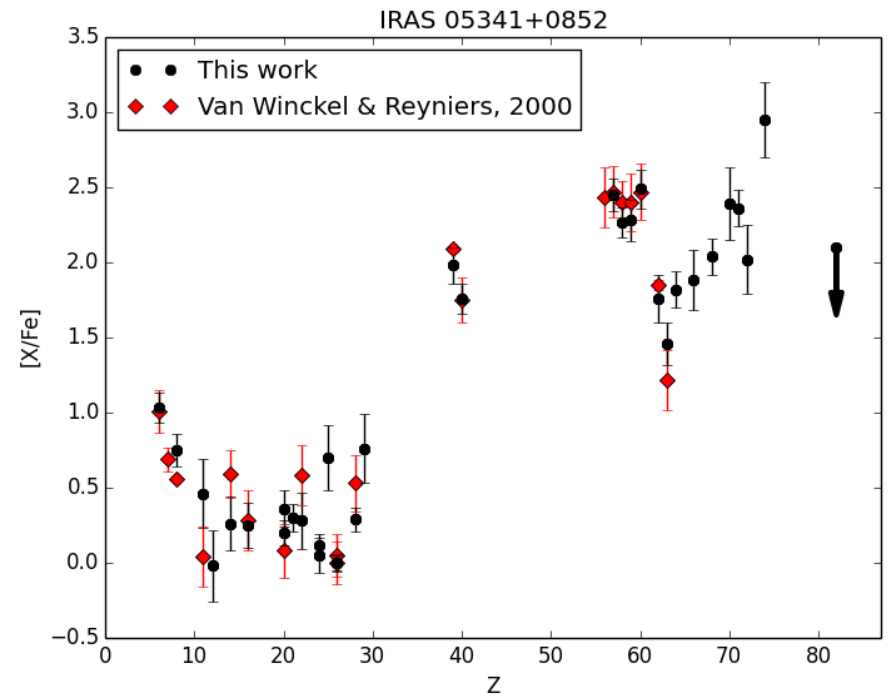

Fig. B.3. Comparison between our derived abundances and the results of Van Winckel \& Reyniers (2000) for IRAS 05341+0852.

ular, Pr and Sm differ strongly, by almost 0.8 dex. For Sm, the same occurs with IRAS $22272+5435$, for which Reddy et al. (2002) find an abundance that is about 1.25 dex higher than our Sm abundance. For all other elements, the abundance differences are significantly lower. Given the consistent distribution of the abundances presented here, and the superior $\mathrm{S} / \mathrm{N}$ of our UVES spectra, we evaluate our new abundances to be more reliable.

For IRAS 05341+0852, IRAS 07134+1005, IRAS 195001709, and IRAS 22223+4327, we find similar atmospheric parameters as Van Winckel \& Reyniers (2000). The strongest difference is the derived temperature of IRAS $05341+0852$, which is $250 \mathrm{~K}$ hotter in our study. We find similar results for IRAS 05341+0852 (Fig. B.3) and IRAS 07134+1005 (Fig. B.4), and only our Sm result differs by about 0.4 dex from that of Van Winckel \& Reyniers (2000). For IRAS 19500-1709 (Fig. B.5), all results are similar except for $\mathrm{Nd}$ and $\mathrm{Eu}$, for which we find abundances that are a remarkable 0.9 dex higher. The large differences in those two elements are attributed to the use of a different set of spectral lines to determine the abundances. Van Winckel \& Reyniers (2000) used only one very small line 


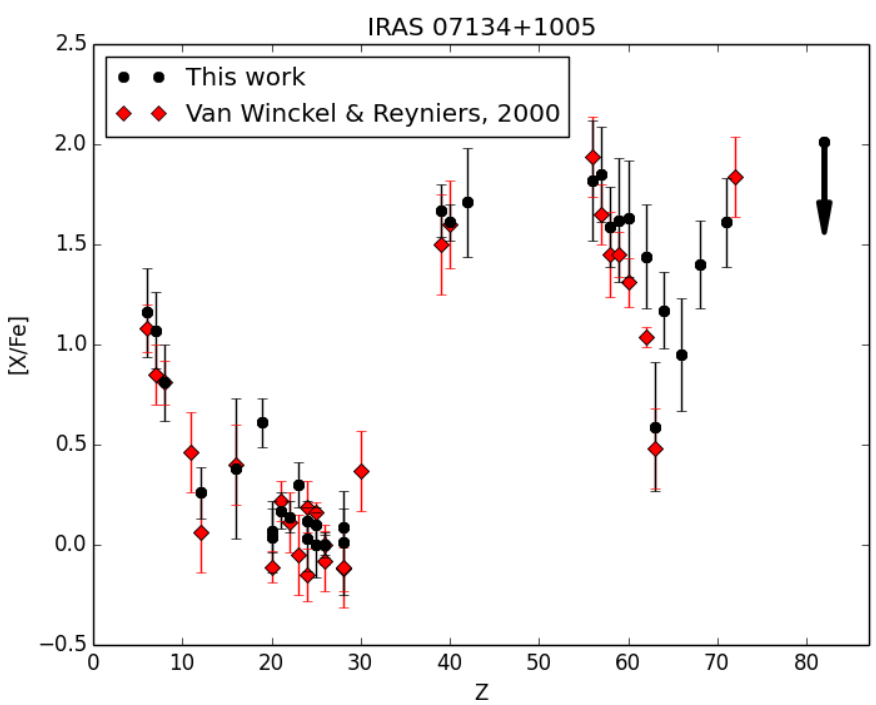

Fig. B.4. Comparison between our derived abundances and the results of Van Winckel \& Reyniers (2000) for IRAS 07134+1005.

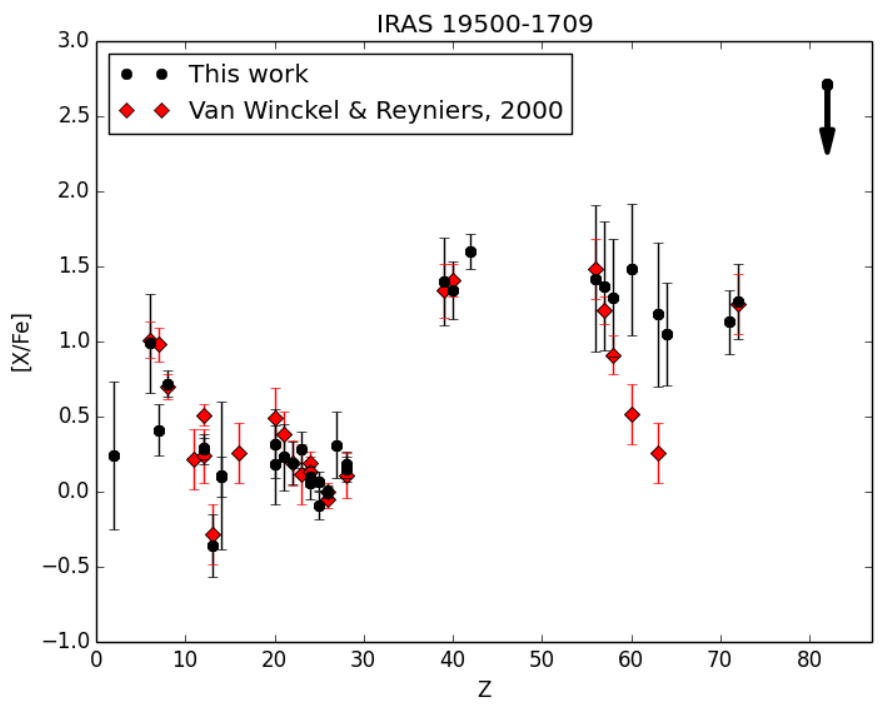

Fig. B.5. Comparison between our derived abundances and the results of Van Winckel \& Reyniers (2000) for IRAS 19500-1709.

in the blue $(\lambda 3863.408)$ with a measured $E W \approx 3 \mathrm{~m} \AA$ to probe the Nd abundance and also used a line of $3 \mathrm{~m} \AA$ for the Eu abundance. The analyses of $\mathrm{Nd}$ presented here is based on five lines with EWs ranging from about 5 up to $24 \mathrm{~m} \AA$. We devised a much more systematic approach to our earlier work and find the low Nd abundance of our earlier work, Van Winckel \& Reyniers (2000), to be suspicious given the very small and maybe spurious line. Also, in our new abundance of Eu presented here, the value is only based on one line; this is different than our earlier work with an $E W \approx 16 \mathrm{~m} \AA$.

Our abundance results of IRAS $22223+4327$ (Fig. B.6) are similar to the determined abundances from Van Winckel \& Reyniers (2000) and Rao et al. (2012). All three independent studies confirm the overabundance of ls elements with respect to the hs elements of IRAS $22223+4327$.

Our atmospheric parameter results for IRAS 06530-0213 and IRAS 08143-4406 (Figs. B.7 and B.8) confirm the derived atmospheric parameter sets of Reyniers et al. (2004). Since only the combined $[\mathrm{X} / \mathrm{Fe}]$ results for the two observational settings for IRAS 08143-4406 are provided in Reyniers et al. (2004),

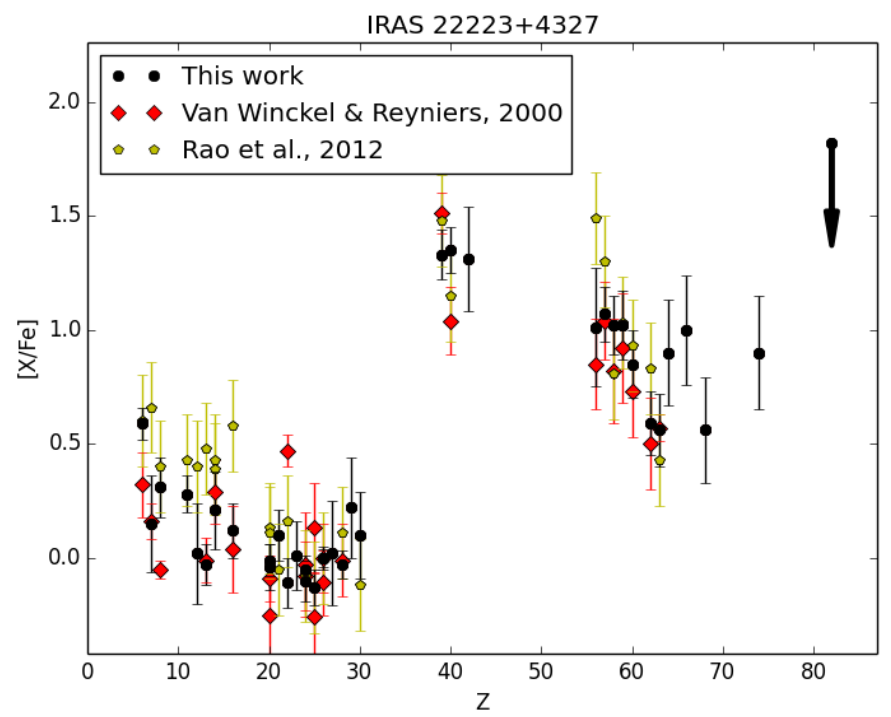

Fig. B.6. Comparison between our derived abundances and the results of Van Winckel \& Reyniers (2000) and Rao et al. (2012) for IRAS $22223+4327$. We adopt a standard deviation of 0.2 dex for all results of Rao et al. (2012).

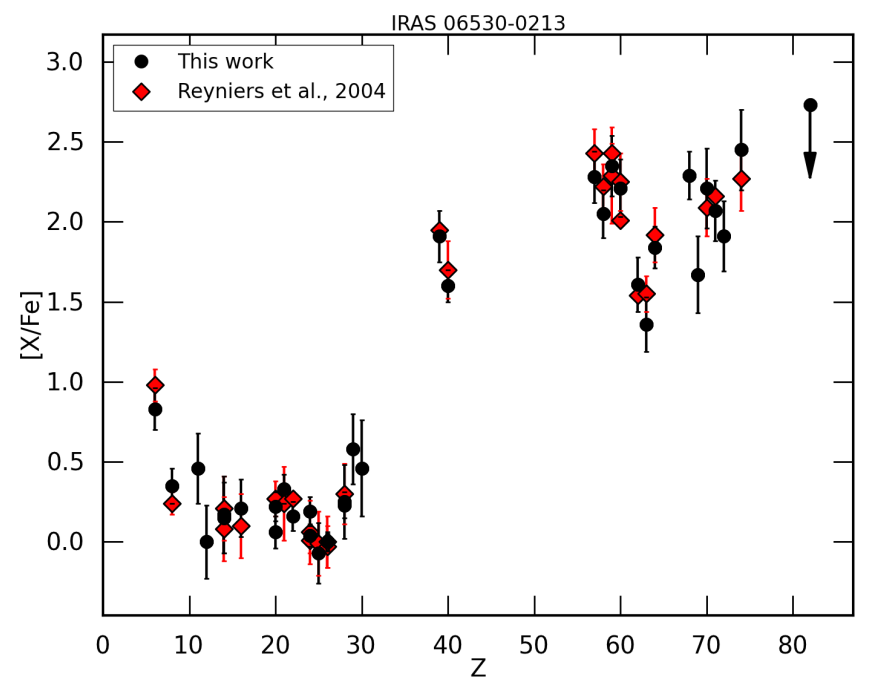

Fig. B.7. Comparison between our derived abundances and the results of Reyniers et al. (2004) for IRAS 06530-0213.

we compare our individual results for the two settings with these combined results. For both stars, our results are similar. The strongest difference is our higher $\mathrm{Zr}$ abundance of about +0.3 dex with respect to Reyniers et al. (2004).

For IRAS 07430+1115 (Fig. B.9), we find large abundance differences with respect to Reddy et al. (1999) for almost all $s$-process elements, even though we used almost exactly the same atmospheric parameter set. Reddy et al. (1999) find Y and $\mathrm{Zr}$ abundances that are \pm 0.5 and \pm 1.0 dex higher than our results, respectively. Reddy et al. (1999) find lower abundances of about 0.3 dex for $\mathrm{Sm}$ and Eu . Except for one Eu line, we have used a different set of spectral lines than Reddy et al. (1999) for the analysis of $\mathrm{Y}, \mathrm{Zr}, \mathrm{Sm}$, and Eu because many of these lines are part of a spectral blend in our UVES spectra. If these blends are included in the EW calculation, they result in EWs that are much higher than the actual EW, which may explain the difference between our abundance results and those of Reddy et al. (1999). For the only common Eu, we find a difference of 


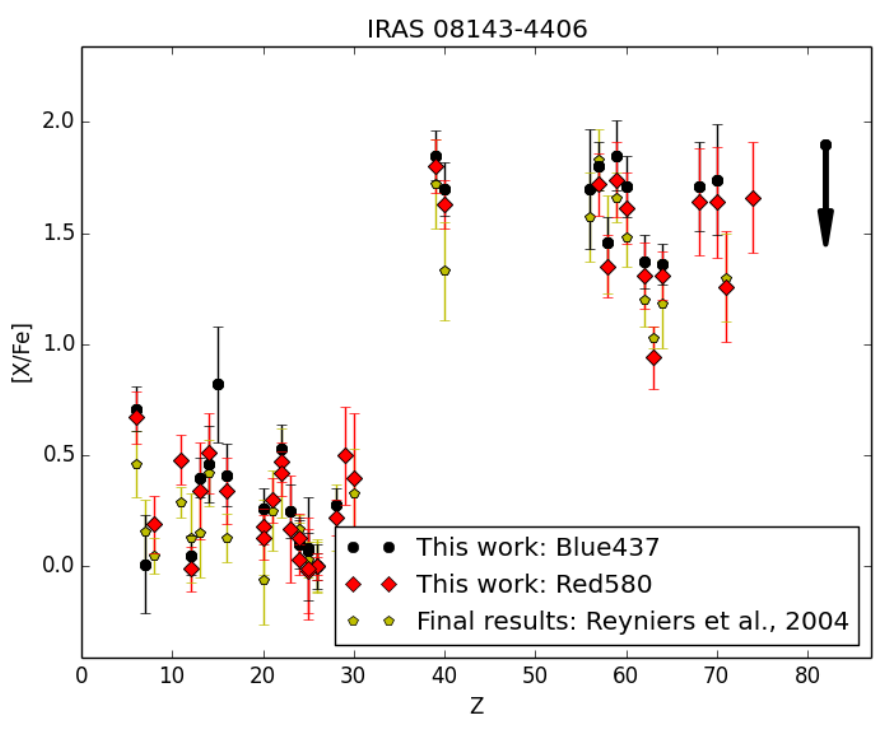

Fig. B.8. Comparison between our derived abundances for both observational settings of IRAS08143-4406 and the final results of Reyniers et al. (2004).

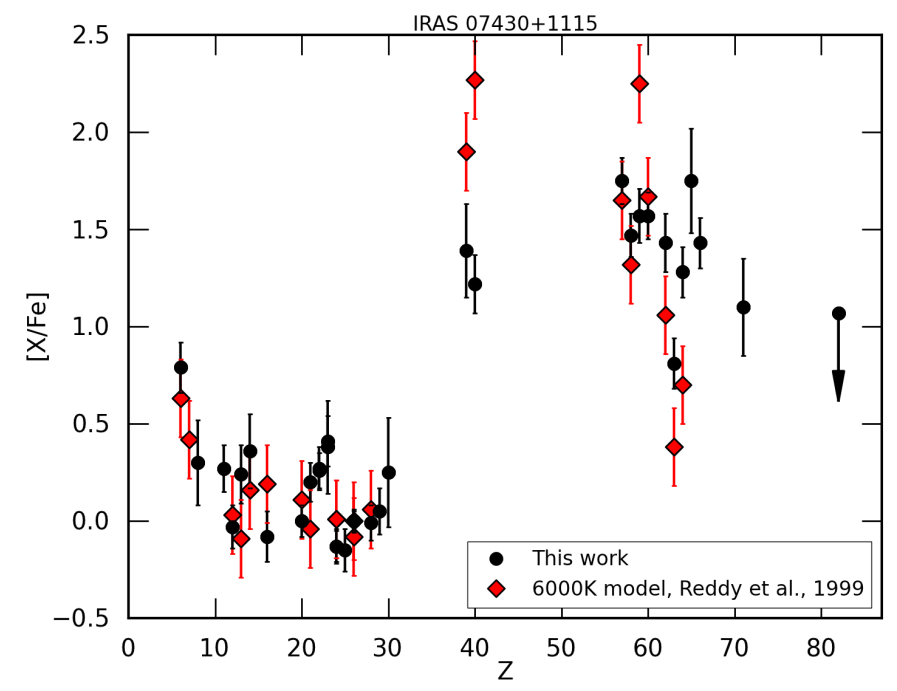

Fig. B.9. Comparison between our derived abundances and the results of Reddy et al. (1999) for IRAS $07430+1115$. We adopt a standard deviation of 0.2 dex for all results of Reddy et al. (1999).

-0.07 dex between our log gf value and those of Reddy et al. (1999), which may partly explain the abundance difference.

The atmospheric parameter results of Reyniers et al. (2007b) for IRAS 08281-4850 and IRAS 14325-6428 are similar to our results, although they find a microturbulent velocity, which is $4 \mathrm{~km} \mathrm{~s}^{-1}$ higher than our preferred result for IRAS 14325-6428. We find similar results for IRAS 08281-4850 (Fig. B.10) and also for IRAS 14325-6428, (Fig. B.11) despite the large microturbulence difference.

As discussed above, our results for IRAS $22223+4327$ correspond to those of Rao et al. (2012). In case of IRAS 172791119 (Fig. B.12), we find similar atmospheric parameters as Rao et al. (2012) except for our $\log g$, which differs by +1.0 dex from that of Rao et al. (2012). We find a stronger enrichment of the hs elements resulting in a mean $[\mathrm{hs} / \mathrm{Fe}]$ of about $0.9 \mathrm{dex}$, while Rao et al. (2012) find [hs/Fe] $\approx 0.6$ dex. Nevertheless, we still classify this object as mildly $s$-process enriched $([\mathrm{s} / \mathrm{Fe}]<1)$ based on our abundance results.

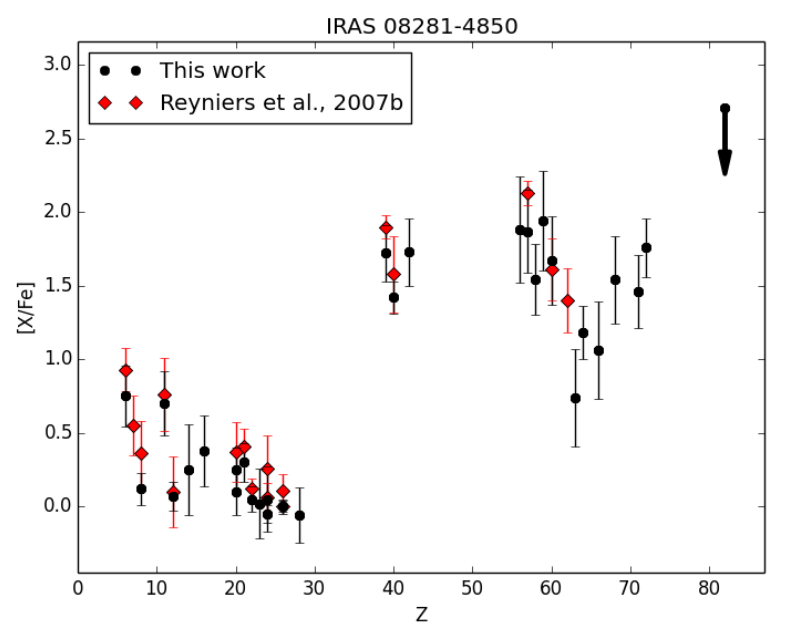

Fig. B.10. Comparison between our derived abundances for both observations of IRAS 08281-4850 and the final results of Reyniers et al. (2007b).

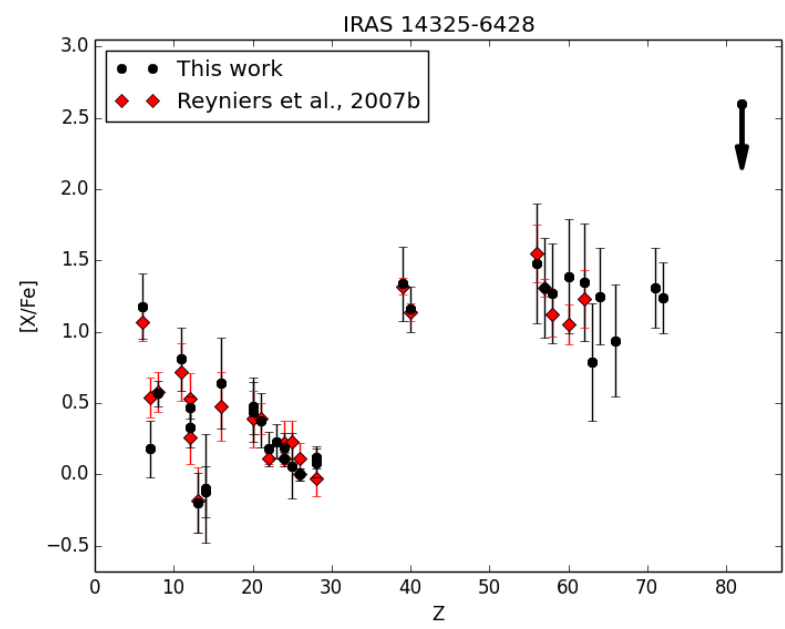

Fig. B.11. Comparison between our derived abundances for both observations of IRAS 14325-6428 and the final results of Reyniers et al. (2007b).

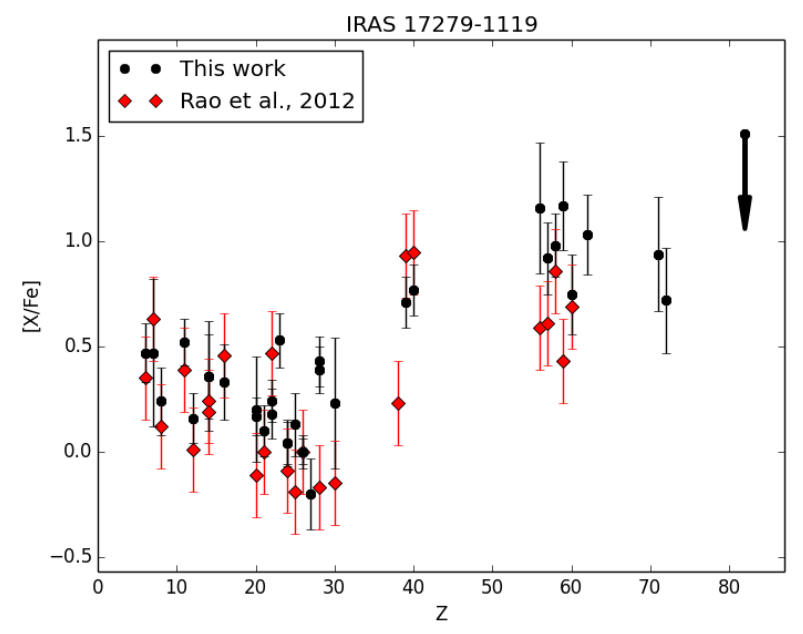

Fig. B.12. Comparison between our derived abundances and the results of Rao et al. (2012) for IRAS 17279-1119. We adopt a standard deviation of 0.2 dex for all results of Rao et al. (2012). 